\title{
Bulk-Boundary Correspondence for Three-Dimensional Symmetry-Protected Topological Phases
}

\author{
Chenjie Wang, ${ }^{1,2}$ Chien-Hung Lin, ${ }^{1,3}$ and Michael Levin ${ }^{1}$ \\ ${ }^{1}$ James Franck Institute and Department of Physics, University of Chicago, Chicago, Illinois 60637, USA \\ ${ }^{2}$ Perimeter Institute for Theoretical Physics, Waterloo, Ontario N2L 2Y5, Canada \\ ${ }^{3}$ Department of Physics, University of Alberta, Edmonton, Alberta T6G 2E1, Canada
}

(Received 6 January 2016; published 9 May 2016)

\begin{abstract}
We derive a bulk-boundary correspondence for three-dimensional (3D) symmetry-protected topological phases with unitary symmetries. The correspondence consists of three equations that relate bulk properties of these phases to properties of their gapped, symmetry-preserving surfaces. Both the bulk and surface data appearing in our correspondence are defined via a procedure in which we gauge the symmetries of the system of interest and then study the braiding statistics of excitations of the resulting gauge theory. The bulk data are defined in terms of the statistics of bulk excitations, while the surface data are defined in terms of the statistics of surface excitations. An appealing property of these data is that it is plausibly complete in the sense that the bulk data uniquely distinguish each 3D symmetry-protected topological phase, while the surface data uniquely distinguish each gapped, symmetric surface. Our correspondence applies to any 3D bosonic symmetryprotected topological phase with finite Abelian unitary symmetry group. It applies to any surface that (1) supports only Abelian anyons and (2) has the property that the anyons are not permuted by the symmetries.
\end{abstract}

DOI: 10.1103/PhysRevX.6.021015

\section{INTRODUCTION}

A gapped quantum many-body system is said to belong to a nontrivial symmetry-protected topological (SPT) phase if it satisfies three conditions. First, the Hamiltonian is invariant under some set of internal (on-site) symmetries, none of which are broken spontaneously. Second, the ground state is short-range entangled-that is, the ground state can be transformed into a product state or atomic insulator using a local unitary transformation. Third, it is impossible to continuously connect the ground state to a product state or atomic insulator, by varying some parameter in the Hamiltonian, without breaking one of the symmetries or closing the energy gap [1-7]. Famous examples of nontrivial SPT phases include the one-dimensional Haldane spin chain [8], which is protected by time-reversal symmetry, and the two-dimensional and three-dimensional topological insulators $[9,10]$ which are protected by timereversal and charge-conservation symmetry.

Perhaps the most interesting property of nontrivial SPT phases is that they have protected boundary modes. Here, the precise meaning of "protected" depends on dimensionality. For example, in the two-dimensional case, the edges of SPT phases are believed to be protected in the sense that

Published by the American Physical Society under the terms of the Creative Commons Attribution 3.0 License. Further distribution of this work must maintain attribution to the author(s) and the published article's title, journal citation, and DOI.
Subject Areas: Condensed Matter Physics,

Strongly Correlated Materials,

Topological Insulators they cannot be both gapped and symmetric [11-16]. On the other hand, in the three-dimensional case, the surfaces of SPT phases are believed to be protected in the sense that any surface that is both gapped and symmetric must also support anyon excitations [17-27].

For some SPT phases, we can not only establish the existence of a protected boundary, but we can derive a "bulkboundary correspondence." Let us clarify what we mean by this term since there are at least two different types of bulkboundary correspondences discussed in the literature. One type of bulk-boundary correspondence is a construction that provides a particular (i.e., nonunique) field theory description of the boundary for each bulk phase [17,28-31]. Another type of bulk-boundary correspondence is a universal relation between measurable properties of the bulk and boundary. In this paper, we are interested in bulk-boundary correspondences of the second kind.

The classic example of such a bulk-boundary correspondence appears in the context of 2D noninteracting fermion systems with charge-conservation symmetry. For these systems, one can relate the bulk electric Hall conductivity $\sigma_{x y}$, measured in units of $e^{2} / h$, to the number $N_{R}, N_{L}$ of right-moving and left-moving edge modes [32]:

$$
\sigma_{x y}=N_{R}-N_{L}
$$

Similar relations, which connect bulk topological band invariants to the properties of boundary modes, are known for other noninteracting fermion systems $[9,10]$. 
Less is known about such bulk-boundary correspondences for interacting SPT phases. One place where it would be particularly useful to have such a correspondence is in the context of 3D SPT phases with gapped symmetric surfaces. This case is interesting because surfaces of this kind are relatively easy to characterize due to the energy gap, but at the same time they exhibit nontrivial structure associated with surface anyon excitations. It is natural to ask, what are the general constraints that relate the bulk and surface properties of these systems?.

There are several cases where this question has been answered - at least partially. In particular, in the case of 3D topological insulators, Refs. [18,33] derived constraints connecting the properties of the surface to properties of monopoles in the bulk. Similarly, it is possible to derive constraints for other 3D SPT phases with at least one U(1) symmetry and one antiunitary symmetry [34]. Unfortunately, however, these constraints rely on a special combination of symmetries and therefore do not give insight into the more general structure of the bulk-boundary correspondence.

In this paper, we take a step towards a more general theory by deriving a bulk-boundary correspondence for a large class of 3D SPT phases. More specifically, we consider general 3D bosonic SPT phases with unitary Abelian symmetries. To simplify the discussion, we focus on gapped symmetric surfaces with the properties that (1) the surface anyons are Abelian and (2) these anyons are not permuted [35-37] by the symmetries. We denote the symmetry group by $G=\prod_{i=1}^{K} \mathbb{Z}_{N_{i}}$, and the group of surface anyons by $A=\prod_{\mu=1}^{M} \mathbb{Z}_{N_{\mu}}$-with the group law in $A$ corresponding to fusion of anyons. For this class of systems, we derive a bulk-boundary correspondence analogous to Eq. (1).

Before we can explain our correspondence, we need to describe the bulk and surface data that we use. The bulk data were originally introduced by Ref. [38] and consist of three tensors,

$$
\text { bulk data }=\left\{\Theta_{i, l}, \Theta_{i j, l}, \Theta_{i j k, l}\right\},
$$

where the indices $i, j, k, l$ range over $1, \ldots, K$. These quantities are defined via a simple recipe. Suppose we are given a lattice boson model belonging to a SPT phase with symmetry group $G$. To find the corresponding bulk data, the first step is to minimally couple the model to a dynamical lattice gauge field with gauge group $G$ [39]. After gauging the model in this way, the second step is to study the braiding statistics of the "vortex loop" excitations of the resulting gauge theory $[40,41]$. The tensors $\left\{\Theta_{i, l}, \Theta_{i j, l}, \Theta_{i j k, l}\right\}$ are then defined in terms of the braiding statistics of these vortex loops, as reviewed in more detail in Sec. II.

The surface data have a similar character and consist of five tensors,

$$
\text { surface data }=\left\{\Phi_{\mu}, \Phi_{\mu \nu}, \Omega_{i \mu}, \Omega_{i j \mu}, x_{i l}^{\mu}\right\},
$$

where the indices $i, j, l$ range over $1, \ldots, K$ and the indices $\mu, \nu$ range over $1, \ldots, M$. Like the bulk data, the surface data are defined by gauging the lattice boson model and studying the braiding statistics of the excitations of the resulting gauge theory. The only difference is that we consider the braiding statistics of surface excitations instead of bulk excitations. In particular, the tensors $\left\{\Phi_{\mu}, \Phi_{\mu \nu}, \Omega_{i \mu}, \Omega_{i j \mu}, x_{i l}^{\mu}\right\}$ are defined in terms of the braiding statistics of surface anyons and vortex lines ending at the surface.

The reason we use the above bulk and surface data is that these data have a number of appealing properties. First, the quantities in Eqs. (2) and (3) are measurable in the sense that they can be extracted from a microscopic model by following a well-defined procedure. Second, these quantities are topological invariants; that is, they remain fixed under continuous, symmetry-preserving deformations of the (ungauged) Hamiltonian that do not close the bulk or surface gap, respectively [42]. Finally, there is reason to think that the bulk data and surface data are complete in the sense that the bulk data uniquely distinguish every 3D SPT phase, while the surface data uniquely distinguish every gapped symmetric surface (we discuss the evidence for this claim in Secs. II B and III C).

The main result of this paper is a set of three equations [Eqs. (30)-(32)] that connect the bulk data Eq. (2) to the surface data Eq. (3). We derive these equations by relating the bulk braiding processes that define Eq. (2) to the surface braiding processes that define Eq. (3) using topological invariance and other properties of braiding statistics.

Our results are closely related to a conjecture of Chen et al. [20]. To describe this conjecture, we need to recall two facts. The first fact is that many (possibly all) 3D SPT phases with finite unitary symmetry group $G$ can be realized by exactly soluble lattice boson models known as group cohomology models [7]. These models are parametrized by elements of the cohomology group $H^{4}(G, \mathrm{U}(1))$. The second fact is that each 2D anyon system with unitary symmetry group $G$ is associated with an anomaly that takes values in $H^{4}(G, \mathrm{U}(1))[20,43]$. (See Refs. [17,44-46] for other related discussions of anomalies.) If this anomaly is nonzero, then the corresponding anyon system cannot be realized in a strictly 2D lattice model. Given these two facts, Chen et al. conjectured that gapped symmetric surfaces of the group cohomology model always have an anomaly $\nu$ that matches the $\nu \in$ $H^{4}(G, \mathrm{U}(1))$ defining the bulk cohomology model. The authors checked that this conjecture gives correct predictions for a particular lattice model.

What is the relationship between our bulk-boundary correspondence and this conjecture? To make a connection, we use our bulk-boundary formulas [Eqs. (30)-(32)] to obtain constraints on the surfaces of group cohomology 
models. We then compare these constraints to those predicted by the conjecture and we show that the two sets of constraints are mathematically equivalent. Thus, our bulk-boundary correspondence gives a proof of the conjecture for the case where $G$ is Abelian. Conversely, the conjecture implies our bulk-boundary correspondence, if we assume that the group cohomology models realize every possible 3D SPT phase.

The rest of the paper is organized as follows. In Secs. II and III, we define the bulk data and surface data, respectively. In Sec. IV, we present the bulk-boundary correspondence [Eqs. (30)-(32)] that relates the two sets of data to one another. We derive the correspondence in Sec. V. We discuss the implications of the correspondence for purely 2D systems in Sec. VI. In Sec. VII, we explain the connection between our bulk-boundary correspondence and the conjecture of Chen et al. [20]. Technical arguments and calculations are given in the appendixes.

\section{BULK DATA: REVIEW}

In this section, we review the definition of the bulk data $\left\{\Theta_{i, l}, \Theta_{i j, l}, \Theta_{i j k, l}\right\}$ [38]; that is, we explain how to compute these quantities given an arbitrary 3D lattice boson model belonging to a SPT phase with unitary Abelian symmetry group $G=\prod_{i=1}^{K} \mathbb{Z}_{N_{i}}$.

As discussed in the Introduction, the computation and definition proceeds in two steps. The first step is to minimally couple the lattice boson model of interest to a dynamical lattice gauge field with gauge group $G$ [39]. The details of this gauging procedure are not important for our purposes; the only requirement is that the gauge coupling constant is small so that the resulting gauge theory is gapped and deconfined. (See Refs. [15,38] for a precise gauging prescription in which the coupling constant is chosen to be exactly zero.) The second step is to study the excitations of the gauged model. The bulk data are defined in terms of the braiding statistics of these excitations.

In what follows, we focus on the second step of this procedure. First, we discuss the excitations of the gauged models and review their braiding statistics. After this preparation, we give the precise definition of $\left\{\Theta_{i, l}\right.$, $\left.\Theta_{i j, l}, \Theta_{i j k, l}\right\}$.

\section{A. Bulk excitations}

We begin by reviewing the excitation spectrum of the gauged models. The gauged models support two types of excitations in the bulk: particlelike charges and looplike vortices. Charge excitations are characterized by their gauge charge

$$
q=\left(q_{1}, \ldots, q_{K}\right),
$$

where each component $q_{i}$ is an integer defined modulo $N_{i}$. Similarly, vortex loop excitations are characterized by their gauge flux

$$
\phi=\left(\phi_{1}, \ldots, \phi_{K}\right)
$$

where each component $\phi_{i}$ is a multiple of $\left(2 \pi / N_{i}\right)$ and is defined modulo $2 \pi$. An important point is that while charge excitations are uniquely characterized by the amount of gauge charge that they carry, there are typically many topologically distinct types of vortex loops that carry the same gauge flux $\phi$. These different loops can be obtained from one another by attaching charge excitations.

Some comments on notation: we use greek letters $\alpha, \beta$, etc. to denote vortex excitations, and we use $\phi_{\alpha}$ to denote the amount of gauge flux carried by $\alpha$. We use $q, q^{\prime}, \ldots$ to denote charge excitations, and we use the same symbols $q, q^{\prime}, \ldots$ to denote the amount of gauge charge that they carry.

We now discuss the braiding statistics of these excitations. There are several types of braiding processes one can consider: (i) braiding of two charges, (ii) braiding of a charge around a vortex loop, and (iii) braiding involving multiple vortex loops. The first kind of braiding process is not very interesting because the charges are all bosons: one way to see this is to note that the charges can be viewed as local excitations of the original ungauged model, and the ungauged model has only bosonic excitations since it is short-range entangled. As for the second kind of process, it is easy to see that if we braid a charge $q$ around a vortex loop $\alpha$, the resulting statistical phase is given by the Aharonov-Bohm law

$$
\theta=q \cdot \phi_{\alpha}
$$

where the centered dot denotes the vector inner product.

All that remains is the last type of braiding process, involving multiple loops. There are several ways to braid vortex loops, but in this paper we are primarily interested in a braiding process in which a loop $\alpha$ is braided around a loop $\beta$ while both loops are linked to a "base loop" $\sigma$ (Fig. 1) $[40,41]$. We also consider more general braiding processes involving multiple loops $\alpha_{1}, \alpha_{2}, \ldots, \alpha_{N}$, all of which are linked to a single base loop $\sigma$. The reason we focus on these kinds of braiding processes is that the associated Berry phases are not fixed by the AharonovBohm law, but instead probe more interesting properties of the bulk.

One technical complication is that in some models the vortex loop excitations have non-Abelian braiding statistics (a)



(b)

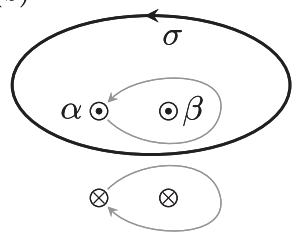

FIG. 1. (a) Three-loop braiding process. (b) Cross section of the braiding process in the plane containing the base loop $\sigma$. 
even though the gauge group $G$ is Abelian. Therefore, even if we specialize to the case where $G$ is Abelian, as we do here, it is still important to have a more complete theory of loop braiding statistics that includes the concepts of fusion rules, quantum dimensions, and so on.

Fortunately, this formalism can be developed rather easily. The key point is that there is a direct analogy between 3D loop braiding and 2D particle braiding. This analogy can be seen by considering a $2 \mathrm{D}$ cross section of the loop braiding process [Fig. 1(b)]. We can see that a braiding process involving two loops linked with a single base loop can be mapped onto a process involving two particles in two dimensions. More generally, a process involving $N$ loops linked to a single base loop can be mapped into a process involving $N$ particles in two dimensions. In addition to braiding, the analogy also carries over to fusion processes. Just as two particles can be fused together to form another particle, two loops $\alpha, \beta$ that are linked to the same loop $\sigma$ can be fused to form a new loop that is also linked to $\sigma$.

With this analogy, we can immediately generalize the notation and results of 2D anyon theory [47] to 3D loop braiding. In particular, we can define $F$ symbols, $R$ symbols, and quantum dimensions of loops in the same way as for particles. We denote these quantities by $F_{\alpha \beta \gamma, c}^{\delta}$, $R_{\alpha \beta, c}^{\delta}$, and $d_{\alpha, c}$. Here, $\alpha, \beta, \gamma$ are loops linked with a base loop $\sigma$, while $c$ is an integer vector that parametrizes the gauge flux carried by $\sigma$ :

$$
\phi_{\sigma}=\left(\frac{2 \pi}{N_{1}} c_{1}, \ldots, \frac{2 \pi}{N_{K}} c_{K}\right) .
$$

(The reader may wonder why we use $c$ instead of $\sigma$ in our notation for $F_{\alpha \beta \gamma, c}^{\delta}, R_{\alpha \beta, c}^{\delta}$, etc. The reason is that these quantities depend on only the gauge flux carried by $\sigma$, and this gauge flux is conveniently parametrized by $c$.)

An additional quantity that we need below is the topological spin of a loop $\alpha$ linked to a base loop $\sigma$. We denote this quantity by $s_{\alpha, c}$, where $0 \leq s_{\alpha, c}<1$. Here, $s_{\alpha, c}$ is defined in the same way as for $2 \mathrm{D}$ anyon theories:

$$
e^{i 2 \pi s_{\alpha, c}}=\frac{1}{d_{\alpha, c}} \sum_{\delta} d_{\delta, c} \operatorname{Tr}\left(R_{\alpha \alpha, c}^{\delta}\right),
$$

where the summation runs over all fusion channels $\delta$ of two $\alpha$ loops, both of which are linked to $\sigma$.

\section{B. Definition of bulk data}

With this preparation, we are now ready to define the bulk data. These data consist of three tensors $\left\{\Theta_{i, l}, \Theta_{i j, l}, \Theta_{i j k, l}\right\}$, where the indices $i, j, k, l$ range over $1, \ldots, K$. To define these tensors, let $\alpha, \beta, \gamma$ be vortex loops linked to a base loop $\sigma$. Suppose that $\alpha$ carries unit type- $i$ flux; that is $\phi_{\alpha}=\left(2 \pi / N_{i}\right) \mathbf{e}_{i}$, where $\mathbf{e}_{i}$ is the vector
$(0, \ldots, 1, \ldots, 0)$ with the $i$ th entry being 1 and all other entries being 0 . Similarly, suppose that $\beta, \gamma, \sigma$ carry unit flux $\left(2 \pi / N_{j}\right) \mathbf{e}_{j},\left(2 \pi / N_{k}\right) \mathbf{e}_{k},\left(2 \pi / N_{l}\right) \mathbf{e}_{l}$, respectively. Then, $\Theta_{i, l}, \Theta_{i j, l}, \Theta_{i j k, l}$ are defined as follows:

(1) $\Theta_{i, l}=2 \pi N_{i} s_{\alpha, \mathbf{e}_{l}}$, where $s_{\alpha, \mathbf{e}_{l}}$ is the topological spin of $\alpha$ when it is linked to $\sigma$;

(2) $\Theta_{i j, l}$ is the Berry phase associated with braiding the loop $\alpha$ around $\beta$ for $N^{i j}$ times, while both are linked to $\sigma$;

(3) $\Theta_{i j k, l}$ is the Berry phase associated with the following braiding process: $\alpha$ is first braided around $\beta$, then around $\gamma$, then around $\beta$ in the opposite direction, and finally around $\gamma$ in the opposite direction. Here, $\alpha, \beta, \gamma$ are all linked to $\sigma$.

Above, we use $N^{i j}$ to denote the least common multiple of $N_{i}$ and $N_{j}$. (Throughout this paper, we use $N^{i j \ldots k}$ and $N_{i j \ldots k}$ to denote the least common multiple and greatest common divisor of integers $N_{i}, N_{j}, \ldots, N_{k}$, respectively.)

These definitions deserve a few comments. First, we point out that one needs to do some work to show that the above quantities are well defined. In particular, one needs to establish two results: (1) $\Theta_{i j, l}, \Theta_{i j k, l}$ are Abelian phases and (2) $\Theta_{i, l}, \Theta_{i j, l}, \Theta_{i j k, l}$ depend only on $i, j, k, l$ and not on the choice of the loops $\alpha, \beta, \gamma, \sigma$. The first fact is not obvious since vortex loops can have non-Abelian braiding statistics in some cases so that the Berry phase associated with general braiding processes is actually non-Abelian. The second fact is not obvious either since there are multiple topologically distinct loop excitations that carry the same gauge flux [48]. The proof of these two properties is given in Ref. [38].

We should also explain our motivation for using this data to characterize bulk SPT phases. Much of our motivation comes from a result of Ref. [38], which showed that $\left\{\Theta_{i, l}, \Theta_{i j, l}, \Theta_{i j k, l}\right\}$ take different values in every group cohomology model with symmetry group $G=\prod_{i} \mathbb{Z}_{N_{i}}$. What makes this result especially interesting is that it has been conjectured that the group cohomology models realize every 3D SPT phase [7]. If this conjecture is true, then we can conclude that the above data are complete in the sense that they can distinguish all 3D SPT phases with finite Abelian unitary symmetry.

Finally, we make a comment about the definition of $\Theta_{i, l}$. Unlike the other two quantities which are concretely defined through braiding processes, $\Theta_{i, l}$ is a rather abstract quantity since $s_{\alpha, c}$ does not have a simple interpretation in terms of physical processes. Fortunately, there is an alternative definition of $\Theta_{i, l}$ that is more concrete [38]:

(1) $\Theta_{i, l}$ is equal to $(-1)$ times the phase associated with braiding $\alpha$ around its antivortex $\bar{\alpha}$ for $N_{i} / 2$ times, while both $\alpha$ and $\bar{\alpha}$ are linked to $\sigma$.

Obviously, this definition fails when $N_{i}$ is odd since $N_{i} / 2$ is not an integer. However, when $N_{i}$ is odd, there is no need to find an alternative definition for $\Theta_{i, l}$ : the reason is that $\Theta_{i, l}$ is known to satisfy the constraints (see Appendix G 2) 


$$
N_{i} \Theta_{i, l}=0 \quad(\bmod 2 \pi), \quad 2 \Theta_{i, l}=\Theta_{i i, l} \quad(\bmod 2 \pi) .
$$

Together with another constraint $N_{i} \Theta_{i i, l}=0(\bmod 2 \pi)$, it is easy to see that these constraints completely determine $\Theta_{i, l}$ in terms of $\Theta_{i i, l}$ when $N_{i}$ is odd. More specifically, one can derive the following relation:

$$
\Theta_{i, l}=\frac{N_{i}+1}{2} \Theta_{i i, l} \quad(\bmod 2 \pi) .
$$

\section{SURFACE DATA}

Here, we define the surface data $\left\{\Phi_{\mu}, \Phi_{\mu \nu}, \Omega_{i \mu}, \Omega_{i j \mu}, x_{i l}^{\mu}\right\}$. Our task is as follows: suppose we are given a 3D lattice boson model belonging to a SPT phase with symmetry group $G=\prod_{i=1}^{K} \mathbb{Z}_{N_{i}}$. Suppose that this model is defined in a geometry with a surface and that this surface has three properties: (1) it is gapped and symmetric, (2) it supports only Abelian anyons, and (3) it has the property that the surface anyons are not permuted by the symmetries. Given a microscopic model of this kind, we need to explain how to compute the corresponding surface data.

The computation and definition proceeds in the same way as for the bulk data. First, we couple the lattice boson model to a dynamical lattice gauge field with gauge group $G$. Then, after gauging the model, we study the surface excitations of the resulting gauge theory. The surface data are defined in terms of the braiding statistics of these surface excitations.

In what follows, we build up to the definition of $\left\{\Phi_{\mu}, \Phi_{\mu \nu}, \Omega_{i \mu}, \Omega_{i j \mu}, x_{i l}^{\mu}\right\}$ in several steps. First, we discuss the surface excitations of the original ungauged boson models. Then, we discuss the excitations of the gauged models. Finally, after this preparation, we explain the definition of the surface data.

\section{A. Surface excitations of ungauged models}

By assumption, the surfaces of the ungauged boson models only support Abelian anyons. The purpose of this section is to introduce some notation for labeling these anyons and describing their braiding statistics.

Our notation for labeling the anyons is based on the observation that the anyons form an Abelian group under fusion. Denoting this Abelian group by $A=\prod_{\mu=1}^{M} \mathbb{Z}_{N_{\mu}}$, we label each anyon by a group element $x \in A$, or equivalently, an $M$-component integer vector

$$
x=\left(x^{1}, \ldots, x^{M}\right),
$$

where each component $x^{\mu}$ is defined modulo $N_{\mu}$. The vacuum anyon $\mathbb{1}$ corresponds to the zero vector $(0, \ldots, 0)$, while the fusion product of two anyons $y, y^{\prime}$ is given by $y+y^{\prime}$.

To describe the braiding statistics of these particles, we focus on the unit anyons - that is, the anyons labeled by vectors $\boldsymbol{\epsilon}_{\mu}=(0, \ldots, 1, \ldots, 0)$ with the $\mu$ th entry being 1 and all other entries being 0 . We denote the exchange statistics of the unit anyon $\boldsymbol{\epsilon}_{\mu}$ by $\Phi_{\mu}$, and we denote the mutual statistics of the unit anyons $\boldsymbol{\epsilon}_{\mu}$ and $\boldsymbol{\epsilon}_{\nu}$ by $\Phi_{\mu \nu}$. From $\Phi_{\mu}$ and $\Phi_{\mu \nu}$, we can reconstruct the exchange statistics $\theta_{x}$ and mutual statistics $\theta_{x y}$ of any anyons $x, y \in A$ :

$$
\begin{gathered}
\theta_{x}=\sum_{\mu}\left(x^{\mu}\right)^{2} \Phi_{\mu}+\sum_{\mu<\nu} x^{\mu} x^{\nu} \Phi_{\mu \nu}, \\
\theta_{x y}=\sum_{\mu \nu} x^{\mu} y^{\nu} \Phi_{\mu \nu} .
\end{gathered}
$$

Here, the above formulas [Eqs. (12) and (13)] follow immediately from the linearity relations for Abelian statistics:

$$
\begin{aligned}
\theta_{x+y} & =\theta_{x}+\theta_{y}+\theta_{x y}, \\
\theta_{x\left(y+y^{\prime}\right)} & =\theta_{x y}+\theta_{x y^{\prime}} .
\end{aligned}
$$

\section{B. Surface excitations of gauged models}

We now move on to discuss the surface excitations in the gauged models. The gauged models support three types of excitations on or near the surface: (1) pointlike charge excitations that can exist in either the bulk or the surface, (2) pointlike surface anyons that are confined to the surface, and (3) vortex line excitations that end on the surface. See Fig. 2 for a sketch of these excitations.

Let us discuss each of these excitations in turn. We begin with the charges and vortex lines. Like their bulk counterparts, the charges can be labeled by their gauge charge $q=\left(q_{1}, \ldots, q_{K}\right)$ while the vortex lines can be labeled by their gauge flux $\phi=\left(\phi_{1}, \ldots, \phi_{K}\right)$. Just as in the bulk, the gauge charge uniquely characterizes the charge excitations, while the gauge flux does not uniquely characterize the vortex lines: there are multiple topologically distinct vortex lines that carry the same gauge flux $\phi$, which differ from one another by the attachment of surface anyons and charges.

There are three types of braiding processes we can perform with charges and vortex lines. First, we can braid or exchange charges with one another. These processes are not very interesting since the charges are all bosons. Second,



FIG. 2. Sketch of excitations on and near the surface. Above the surface is the gauged SPT model and below is the vacuum. 
we can braid charges around vortex lines. As in the bulk, the Berry phase for such processes is given by the AharonovBohm formula Eq. (6). Finally, we can braid vortex lines around one another [49]. The latter processes are highly nontrivial and can even give non-Abelian Berry phases. We see some examples of these processes later when we derive the bulk-boundary correspondence in Sec. V.

Let us now turn to the surface anyons. To understand the structure of these excitations, it is helpful to think about their relationship to the surface anyons in the ungauged models. In particular, we argue below that each surface anyon $\mathcal{X}$ in the gauged model is naturally associated with a corresponding surface anyon in the ungauged model, which we denote by $\xi_{\mathcal{X}}$. We think of the mapping $\mathcal{X} \rightarrow \xi_{\mathcal{X}}$ as being analogous to the mapping between vortices $\alpha$ and their gauge flux $\phi_{\alpha}$; therefore, we use the terminology that $\xi_{\mathcal{X}}$ is the "anyonic flux" carried by $\mathcal{X}$. Like the gauge flux, $\xi_{\mathcal{X}}$ can be concretely represented as a vector-or, more precisely, an $M$-component integer vector, as in Eq. (11).

At an intuitive level, $\xi_{\mathcal{X}}$ is obtained by "ungauging" the excitation $\mathcal{X}$; to define $\xi_{\mathcal{X}}$ more precisely, let $|\tilde{\psi}\rangle$ be an excited state of the gauged model which contains an anyon $\mathcal{X}$ localized near some point $r$. Let us suppose that $|\tilde{\psi}\rangle$ has vanishing gauge flux through every plaquette in the lattice. (We can make this assumption without any loss of generality since $\mathcal{X}$ is not a vortex excitation.) Then we can find a gauge in which the state $|\tilde{\psi}\rangle$ has a vanishing lattice gauge field on every link of the lattice. In this gauge, the state $|\tilde{\psi}\rangle$ can be written as a tensor product $|\tilde{\psi}\rangle=|\psi\rangle \otimes|a=0\rangle$, where $|a=0\rangle$ is a ket describing the configuration of the lattice gauge fields, and $|\psi\rangle$ is a ket describing the configuration of the matter fields. Since $|\psi\rangle$ involves only matter fields, we can think of it as an excited state of the ungauged model. By construction, this state contains a localized excitation near the point $r$; we define $\xi_{\mathcal{X}}$ to be the anyon type of this localized excitation [50].

The mapping between anyons $\mathcal{X}$ and their anyonic flux $\xi_{\mathcal{X}}$ has several important properties. The first property is that the mapping is not one to one: distinct anyons $\mathcal{X} \neq \mathcal{X}^{\prime}$ can carry the same anyonic flux, $\xi_{\mathcal{X}}=\xi_{\mathcal{X}^{\prime}}$. The simplest example of this is given by the charge excitations: all the charges share the same (trivial) flux, $\xi_{q}=(0, \ldots, 0)$. More generally, two anyons $\mathcal{X}, \mathcal{X}^{\prime}$ have the same anyonic flux if and only if $\mathcal{X}^{\prime}$ can be obtained from $\mathcal{X}$ by attaching a charge excitation; that is, $\mathcal{X}^{\prime}=\mathcal{X} \times q$ for some $q$.

Another important property of the mapping $\mathcal{X} \rightarrow \xi_{\mathcal{X}}$ is that the braiding statistics of anyons $\mathcal{X}, \mathcal{Y}$, etc. is identical to the braiding statistics of $\xi_{\mathcal{X}}, \xi_{\mathcal{Y}}$, etc. More specifically, it can be shown that the mutual statistics between any two anyons $\mathcal{X}$ and $\mathcal{Y}$ is Abelian and is given by

$$
\theta_{\mathcal{X Y}}=\theta_{\xi_{\mathcal{X} \xi_{\mathcal{Y}}}}
$$

Similarly, the exchange statistics of $\mathcal{X}$, or, equivalently, its topological spin $2 \pi s_{\mathcal{X}}$, is given by

$$
2 \pi s_{\mathcal{X}}=\theta_{\mathcal{X}}=\theta_{\xi_{\mathcal{X}}} .
$$

Given these results, one might be tempted to conclude that the surface anyons in the gauged model have Abelian statistics. However, this conclusion is incorrect: the surface anyons in the gauged model can be non-Abelian in general. This non-Abelian character is not manifest when we focus on braiding processes that involve only surface anyons, but it becomes apparent when we consider processes in which a surface anyon is braided around a vortex line. Such processes will play an important role in the definition of the surface data in the next section.

The last property of the mapping $\mathcal{X} \rightarrow \xi_{\mathcal{X}}$ involves fusion rules. Because the surface anyons can be non-Abelian, they can have complicated fusion rules of the general form

$$
\mathcal{X} \times \mathcal{Y}=\sum_{\mathcal{Z}} N_{\mathcal{X} Y}^{\mathcal{Z}} \mathcal{Z}
$$

However, these rules have a special structure: all the fusion outcomes $\mathcal{Z}$ carry the same anyonic flux $\xi_{\mathcal{Z}}$, which is given by

$$
\xi_{\mathcal{Z}}=\xi_{\mathcal{X}}+\xi_{\mathcal{Y}}
$$

\section{Definition of surface data}

Now that we have discussed the surface excitations and their statistics, we are ready to define the surface data. These data consist of five tensors $\left\{\Phi_{\mu \nu}, \Phi_{\mu}, \Omega_{i \mu}, \Omega_{i j \mu}, x_{i l}^{\mu}\right\}$, where the indices $i, j, l$ run over $1, \ldots, K$ while $\mu, \nu$ run over $1, \ldots, M$. We have already defined $\Phi_{\mu \nu}, \Phi_{\mu}$ in Sec. III A; below, we define the other two quantities $\Omega_{i \mu}$ and $\Omega_{i j \mu}$. We discuss $x_{i l}^{\mu}$ in the next section.

The two quantities $\Omega_{i \mu}$ and $\Omega_{i j \mu}$ are defined in terms of braiding statistics of surface anyons and vortex lines. Let $\mathcal{X}$ be a surface anyon with unit type- $\mu$ anyonic flux; i.e., $\xi_{\mathcal{X}}=\boldsymbol{\epsilon}_{\mu}$, where $\boldsymbol{\epsilon}_{\mu}=(0, \ldots, 1, \ldots, 0)$ with the $\mu$ th entry being 1 . Let $\alpha, \beta$ be vortex lines carrying unit type- $i$ and type- $j$ flux; i.e., $\phi_{\alpha}=\left(2 \pi / N_{i}\right) \mathbf{e}_{i}$ and $\phi_{\beta}=\left(2 \pi / N_{j}\right) \mathbf{e}_{j}$. Then, $\Omega_{i \mu}$ and $\Omega_{i j \mu}$ are defined by the following:

(1) $\Omega_{i \mu}$ is the Berry phase associated with braiding $\mathcal{X}$ around $\alpha$ for $N^{i \mu}$ times;

(2) $\Omega_{i j \mu}$ is the Berry phase associated with the following process: $\mathcal{X}$ is first braided around $\alpha$, then around $\beta$, then around $\alpha$ in the opposite direction, and finally around $\beta$ in the opposite direction.

The above braiding processes are shown in Fig. 3. Similarly to Sec. II B, we use the notation $N^{i \mu}$ to denote the least common multiple of $N_{i}$ and $N_{\mu}$, where the $N_{i}$ are the cyclic factors in the symmetry group $G=\prod_{i=1}^{K} \mathbb{Z}_{N_{i}}$, and the $N_{\mu}$ are the cyclic factors in the anyon group $A=\prod_{\mu=1}^{M} \mathbb{Z}_{N_{\mu}}$.

To show that the above quantities are well defined, we need to prove that (i) $\Omega_{i \mu}, \Omega_{i j \mu}$ are always Abelian phases and (ii) they depend only on the indices $i, j$, $\mu$, i.e., on the gauge flux of the vortex lines and the anyonic flux of $\mathcal{X}$. In 
(a)



(b)

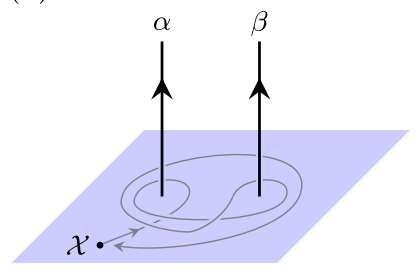

FIG. 3. Braiding processes that define the surface data $\Omega_{i \mu}$ (a) and $\Omega_{i j \mu}$ (b).

other words, if we choose another anyon $\mathcal{X}^{\prime}$ with $\xi_{\mathcal{X}^{\prime}}=\boldsymbol{\epsilon}_{\mu}$ and another pair of vortex lines $\alpha^{\prime}, \beta^{\prime}$ with $\phi_{\alpha^{\prime}}=$ $\left(2 \pi / N_{i}\right) \mathbf{e}_{i}, \phi_{\beta^{\prime}}=\left(2 \pi / N_{j}\right) \mathbf{e}_{j}$, we will obtain the same phases $\Omega_{i \mu}$ and $\Omega_{i j \mu}$. The proof of these two points is technical, and hence is given separately in Appendix A.

Our motivation for using the above surface data is twofold. First, there is reason to think that the surface data are complete in the sense that they can distinguish every gapped symmetric surface satisfying our two assumptions, namely that (1) the surface supports only Abelian anyons and (2) these anyons are not permuted by the symmetry. The main evidence for this is that our data $\left\{\Phi_{\mu \nu}, \Phi_{\mu}, \Omega_{i \mu}, \Omega_{i j \mu}, x_{i l}^{\mu}\right\}$ are mathematically equivalent to another set of data $[36,37,47,51] \quad\left\{F_{x y z}, R_{x y}, \omega \in\right.$ $\left.H^{2}(G, A)\right\}$ that characterizes $2 \mathrm{D}$ anyon systems with symmetry group $G$. We discuss this equivalence in Sec. VII. The latter data are plausibly complete in the context of the above class of surfaces [52], and if this is the case, then our data must be complete as well. Our second source of motivation for using the above surface data is that they can be naturally related to the bulk data via a bulkboundary correspondence, as we discuss below.

\section{Auxiliary surface quantity}

The last piece of surface data is a three index tensor $x_{i l}^{\mu}$, where the indices $i, l$ run from $1, \ldots, K$, while $\mu$ runs from $1, \ldots, M$. We define this quantity implicitly via the equation

$$
\sum_{\nu} x_{i l}^{\nu} \Phi_{\mu \nu}=\Omega_{i l \mu} \quad(\bmod 2 \pi) .
$$

More precisely, we define $x_{i l}^{\nu}$ to be the unique integer tensor that satisfies the above equation and has components $x_{i l}^{\nu} \in\left\{0,1, \ldots, N_{\nu}-1\right\}$.

To understand the physical meaning of Eq. (18), let us imagine fixing the indices $i, l$. Then, $x_{i l}^{\nu}$ reduces to an $M$ component integer vector, which we can think of as describing a surface anyon $x_{i l}$ in the ungauged model. Likewise, the left-hand side of Eq. (18) can be interpreted as the statistical phase associated with braiding a type- $\mu$ unit anyon around the anyon $x_{i l}$. In this interpretation, $x_{i l}$ represents the unique surface anyon with the property that its mutual statistics with a type- $\mu$ unit anyon is $\Omega_{i l \mu}$. From this point of view, it is not hard to see why $x_{i l}^{\mu}$ exists and is unique. Indeed, the uniqueness of $x_{i l}^{\mu}$ follows from the general principle of "braiding nondegeneracy," which guarantees that no two surface anyons have the same mutual statistics with respect to all other surface anyons [47]. As for the existence of $x_{i l}^{\mu}$, this is less obvious but can also be deduced, with some algebra, from braiding nondegeneracy together with the fact that $\Omega_{i l \mu}$ is a multiple of $2 \pi / N_{\mu}$ [see Eq. (24) below].

From Eq. (18) we can see that $x_{i l}^{\mu}$ carries the same information as $\Omega_{i j \mu}$ (assuming we know $\Phi_{\mu \nu}$ ). Therefore, the reader may wonder why we bother to define this additional quantity. One reason is that $x_{i l}^{\mu}$ is often more convenient to work with than $\Omega_{i j \mu}$. Another reason is that the quantity $x_{i l}^{\mu}$ plays an important role in the bulkboundary correspondence. This role can be traced to the following thought experiment. Imagine a configuration of two linked vortex loops, $\alpha$ and $\sigma$ in the bulk (Fig. 4), where $\alpha$ and $\sigma$ carry unit type- $i$ and type- $l$ flux, respectively; that is, $\phi_{\alpha}=\left(2 \pi / N_{i}\right) \mathbf{e}_{i}$ and $\phi_{\sigma}=\left(2 \pi / N_{l}\right) \mathbf{e}_{l}$. Now imagine that we pull the linked loops down to the surface, absorbing a part of each loop into the surface, leaving a pair of tangled vortex "arches" [Fig. 4(b)]. We then disentangle the two arches by unwinding one of the two ends of $\sigma$ [the braiding path is shown in Fig. 4(b)]. The result is two separate arches [Fig. 4(c)]. We then shrink the two separated arches to the surface, leaving behind two localized surface excitations. We denote the resulting state by $|\psi\rangle$. In general, we know that any surface excitation can be written as a linear superposition of different states, each of which has a definite anyon type. Thus, we can write

$$
|\psi\rangle=c|\mathcal{X}\rangle \otimes|\mathcal{S}\rangle+c^{\prime}\left|\mathcal{X}^{\prime}\right\rangle \otimes\left|\mathcal{S}^{\prime}\right\rangle+\cdots,
$$

where $|\mathcal{X}\rangle \otimes|\mathcal{S}\rangle$ denotes a state with surface anyons $\mathcal{X}$ and $\mathcal{S}$, while $\left|\mathcal{X}^{\prime}\right\rangle \otimes\left|\mathcal{S}^{\prime}\right\rangle$ denotes a state with surface anyons $\mathcal{X}^{\prime}$ and $\mathcal{S}^{\prime}$, and the coefficients $c, c^{\prime}$, etc. are the corresponding complex amplitudes [Fig. 4(d)]. What does this thought experiment have to do with $x_{i l}^{\nu}$ ? We show in Appendix B that all the anyons $\mathcal{X}, \mathcal{X}^{\prime}, \ldots$ carry the same anyonic flux $x_{i l}$, while all the anyons $\mathcal{S}, \mathcal{S}^{\prime}, \ldots$ carry anyonic flux $-x_{i l}$.

$$
\begin{aligned}
\xi_{\mathcal{X}} & =\xi_{\mathcal{X}^{\prime}}=\cdots=x_{i l}, \\
\xi_{\mathcal{S}} & =\xi_{\mathcal{S}^{\prime}}=\cdots=-x_{i l} .
\end{aligned}
$$

Thus, the quantity $x_{i l}$ naturally appears when we think about absorbing linked vortex loops into the surface.

\section{E. General constraints on surface data}

In this section, we discuss some of the constraints on $\left\{\Phi_{\mu \nu}, \Phi_{\mu}, \Omega_{i \mu}, \Omega_{i j \mu}, x_{i l}^{\mu}\right\}$. Two important constraints are

$$
N_{\mu \nu} \Phi_{\mu \nu}=0 \quad(\bmod 2 \pi)
$$


(a)

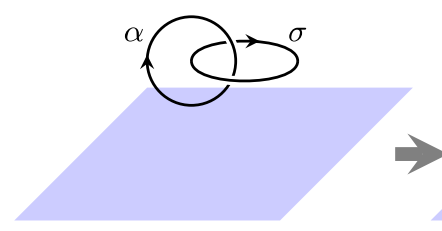

(b) (c) (d)

FIG. 4. Interpreting $x_{i l}^{\mu}$ through a 3D thought experiment. See the main text for details.

and

$$
N_{\mu} \Phi_{\mu}= \begin{cases}0 \quad(\bmod 2 \pi) & \text { if } N_{\mu} \text { is odd } \\ 0 \text { or } \pi \quad(\bmod 2 \pi) & \text { if } N_{\mu} \text { is even. }\end{cases}
$$

To derive these constraints, consider the unit type- $\mu$ anyon $\boldsymbol{\epsilon}_{\mu}$. Using the fact that the fusion product of $N_{\mu}$ of these anyons gives the vacuum excitation, it is not hard to show that $N_{\mu} \Phi_{\mu \nu}=0(\bmod 2 \pi)$ and $N_{\mu}^{2} \Phi_{\mu}=0(\bmod 2 \pi)$. With these relations in hand, the above constraints follow immediately.

Another set of constraints is

$$
\begin{gathered}
N_{i \mu} \Omega_{i \mu}=0 \quad(\bmod 2 \pi), \\
N_{i j \mu} \Omega_{i j \mu}=0 \quad(\bmod 2 \pi), \\
\Omega_{i j \mu}+\Omega_{j i \mu}=0 \quad(\bmod 2 \pi), \\
\Omega_{i i \mu}=0 \quad(\bmod 2 \pi) .
\end{gathered}
$$

We derive these constraints in Appendix G 1. A final set of constraints involve $x_{i l}^{\mu}$ :

$$
\begin{gathered}
N_{i l \mu} x_{i l}^{\mu}=0 \quad\left(\bmod N_{\mu}\right), \\
x_{i l}^{\mu}+x_{l i}^{\mu}=0 \quad\left(\bmod N_{\mu}\right), \\
x_{i i}^{\mu}=0 \quad\left(\bmod N_{\mu}\right) .
\end{gathered}
$$

These constraints follow from Eqs. (24)-(26) together with the definition of $x_{i l}^{\mu}$ [Eq. (18)].

Note there are additional constraints on the surface data beyond those listed above. In particular, the requirement that the surface anyons obey braiding nondegeneracy [47] gives extra constraints on $\Phi_{\mu \nu}$. We do not write out these constraints explicitly since they are not necessary for our purposes.

\section{BULK-BOUNDARY CORRESPONDENCE}

\section{A. The correspondence}

Having defined the bulk data $\left\{\Theta_{i, l}, \Theta_{i j, l}, \Theta_{i j k, l}\right\}$ and the surface data $\left\{\Phi_{\mu \nu}, \Phi_{\mu}, \Omega_{i \mu}, \Omega_{i j \mu}, x_{i l}^{\mu}\right\}$, we are now ready to discuss the connection between the two. This connection is encapsulated by three equations, defined modulo $2 \pi$ :

$$
\begin{aligned}
\Theta_{i, l}= & \sum_{\mu} \frac{N_{i}}{N^{i \mu}} x_{i l}^{\mu} \Omega_{i \mu}+\sum_{\mu} N_{i}\left(x_{i l}^{\mu}\right)^{2} \Phi_{\mu}, \\
\Theta_{i j, l}= & \sum_{\mu}\left(\frac{N^{i j}}{N^{j \mu}} x_{i l}^{\mu} \Omega_{j \mu}+\frac{N^{i j}}{N^{i \mu}} x_{j l}^{\mu} \Omega_{i \mu}\right) \\
& +\frac{N^{i j}\left(N^{i j}-1\right)}{2} \sum_{\mu}\left(x_{j l}^{\mu}-x_{i l}^{\mu}\right) \Omega_{i j \mu}, \\
\Theta_{i j k, l}= & \sum_{\mu}\left(x_{k l}^{\mu} \Omega_{i j \mu}+x_{i l}^{\mu} \Omega_{j k \mu}+x_{j l}^{\mu} \Omega_{k i \mu}\right) .
\end{aligned}
$$

These equations are the main results of this paper. We present their derivation in Sec. V, but before doing that, we make some comments about these formulas and their implications.

(1) Note that the left-hand side of Eqs. (30)-(32) consists of the bulk data $\left\{\Theta_{i, l}, \Theta_{i j, l}, \Theta_{i j k, l}\right\}$, while the right-hand side is built entirely out of the surface data $\left\{\Phi_{\mu \nu}, \Phi_{\mu}, \Omega_{i \mu}, \Omega_{i j \mu}, x_{i l}^{\mu}\right\}$. Thus, these equations allow us to completely determine the bulk data from the surface data. They also provide some constraints on the surface data given the bulk data. This asymmetry between bulk and surface, which is also manifest in Eq. (1), is not surprising, since we expect that a given bulk phase can support many different types of surfaces.

(2) Equations (30)-(32) have an important corollary: any $3 \mathrm{D}$ short-range entangled bosonic model that has nonzero values for $\left\{\Theta_{i, l}, \Theta_{i j, l}, \Theta_{i j k, l}\right\}$, has a protected surface; i.e., its surface cannot be both gapped and symmetric unless it supports anyon excitations. To derive this corollary, we note that if we could find gapped symmetric surface without anyon excitations, then the right-hand side of Eqs. (30)-(32) would vanish for this surface since the sum over $\mu$ would run over the empty set. Clearly this vanishing is inconsistent with nonzero values of $\left\{\Theta_{i, l}, \Theta_{i j, l}, \Theta_{i j k, l}\right\}$, so we conclude that such a surface is not possible.

(3) Another important corollary is that the right-hand side of Eqs. (30)-(32) vanishes for any strictly 2D system. Indeed, we can derive this by thinking of the $2 \mathrm{D}$ system as living on the boundary of a 3D vacuum 
where $\left\{\Theta_{i, l}, \Theta_{i j, l}, \Theta_{i j k, l}\right\}$ are all zero. We discuss this point in more detail in Sec. VI.

(4) It is natural to ask whether there could be additional constraints relating bulk and surface data beyond Eqs. (30)-(32). The analysis in this paper is not capable of answering this question definitively. That being said, if there are additional constraints, we can always replace the bulk data appearing in these constraints with surface data, using Eqs. (30)-(32). Hence, any additional constraints can be written entirely in terms of surface data.

(5) The coefficient of $\Omega_{i \mu}$ in Eq. (30) is an integer. This is not obvious, but can be proven using one of the general constraints on $x_{i l}^{\mu}$, namely, Eq. (27). This integrality property is important because $\Omega_{i \mu}$ is only defined modulo $2 \pi$ : therefore, it is only because its coefficient is an integer that Eq. (30) gives a welldefined phase $\Theta_{i, l}$. In a similar fashion one can check that all the coefficients of the phase factors $\Omega_{i j \mu}, \Phi_{\mu}$ in Eqs. (30)-(32) are integers, so all of these equations are well defined. Finally, one can check that the coefficients of $x_{i l}^{\mu}$ and $\left(x_{i l}^{\mu}\right)^{2}$ in Eqs. (30)(32) satisfy appropriate conditions so that all three equations are well defined even if $x_{i l}^{\mu}$ is defined modulo $N_{\mu}$.

(6) Several of the terms in these equations can take only two values: 0 or $\pi$. In particular, this is the case for the second term on the right-hand side of Eq. (30) as well as the second term on the right-hand side of Eq. (31). This property is interesting because it means that the above equations are simpler than they appear. These results can be established using the general constraints Eqs. (29), (22), and (24).

How can we make use of the above bulk-boundary correspondence? We envision two types of applications. First, if we are given a surface theory and are able to extract all the surface data, we can use the bulk-boundary correspondence to constrain the bulk SPT phase. Conversely, if we are given a bulk SPT phase, we can use the bulk-boundary correspondence to constrain the possible surfaces. Below, we give two examples to illustrate these two ways of applying the bulk-boundary correspondence.

\section{B. Example 1}

In this section, we demonstrate the bulk-boundary correspondence by computing the bulk data corresponding to four different types of surfaces with $\mathbb{Z}_{2} \times \mathbb{Z}_{2}$ symmetry. These surface theories were originally introduced and analyzed by Ref. [20], as we explain below.

To set up our example, imagine that we have a 3D lattice spin model that realizes a SPT phase with a symmetry group $G=\mathbb{Z}_{2} \times \mathbb{Z}_{2}$. Imagine that we study the model in a geometry with a boundary and we find that the surface is gapped and symmetric and that it supports two distinct types of Abelian anyons: a semion $s$ with exchange statistics $\theta_{s}=\pi / 2$ and the vacuum excitation $\mathbb{1}$ with trivial statistics. Translating this information into our notation, this means that the surface anyons form a group $A=\mathbb{Z}_{2}$, while their statistics can be summarized by two quantities:

$$
\Phi_{1}=\pi / 2, \quad \Phi_{11}=\pi .
$$

Here, the index $\mu$ can take only one value-namely, $\mu=1$ - since the group $A=\mathbb{Z}_{2}$ has only one generator.

Next, suppose we couple the system to a $\mathbb{Z}_{2} \times \mathbb{Z}_{2}$ gauge field in order to probe its symmetry properties. After performing this gauging procedure, we take a surface anyon with anyonic flux $s$ and we braid it twice around a vortex line that carries gauge flux $(\pi, 0)$. We find that the Berry phase associated with this process is $\pi$. We also braid the surface anyon twice around the vortex line $(0, \pi)$ and we find a Berry phase of $\pi$. Finally, we braid the surface anyon around one vortex line carrying flux $(\pi, 0)$ and one vortex line carrying flux $(0, \pi)$, then we braid in the opposite direction around both vortex lines as described in Sec. III C and we find that the associated Berry phase is again $\pi$. In our notation, this information is summarized by the surface data:

$$
\Omega_{11}=\pi, \quad \Omega_{21}=\pi, \quad \Omega_{121}=\pi .
$$

Here, the indices $i, j$ can take two values $i, j=1,2$ since the symmetry group $G=\mathbb{Z}_{2} \times \mathbb{Z}_{2}$ has two generators.

To complete the surface data, we still need several more quantities, namely, $\left\{\Omega_{111}, \Omega_{221}, \Omega_{211}\right\}$ and $\left\{x_{12}^{1}, x_{21}^{1}\right.$, $\left.x_{11}^{1}, x_{22}^{1}\right\}$. The first set of quantities can be completely fixed using general properties of $\Omega_{i j \mu}$. In particular, we know that $\Omega_{111}=\Omega_{221}=0$ by Eq. (26), while $\Omega_{211}=-\Omega_{121}$ by Eq. (25). The remaining quantities $\left\{x_{12}^{1}, x_{21}^{1}, x_{11}^{1}, x_{22}^{1}\right\}$ are then completely determined by the definition of $x_{i l}^{\mu}$ [Eq. (18)]:

$$
x_{12}^{1}=x_{21}^{1}=1, \quad x_{11}^{1}=x_{22}^{1}=0 .
$$

With the above surface data in hand, we can now illustrate the bulk-boundary correspondence Eqs. (30)(32). Let us focus on computing the bulk data $\Theta_{1,2}$ and $\Theta_{2,1}$. Substituting the surface data into Eq. (30), we obtain

$$
\begin{aligned}
\Theta_{1,2} & =x_{12}^{1} \Omega_{11}+2\left(x_{12}^{1}\right)^{2} \Phi_{1} \\
& =\pi+\pi \\
& =0
\end{aligned}
$$

and

$$
\begin{aligned}
\Theta_{2,1} & =x_{21}^{1} \Omega_{21}+2\left(x_{21}^{1}\right)^{2} \Phi_{1} \\
& =\pi+\pi \\
& =0 .
\end{aligned}
$$


Similarly, we can go ahead and compute the remaining bulk data, e.g., $\Theta_{1,1}, \Theta_{2,2}, \Theta_{11,2}$, etc., with the result being that they all vanish as well. Alternatively, we can obtain this result using the general constraints Eqs. (G5)-(G14), which completely determine these quantities in terms of $\Theta_{1,2}$ and $\Theta_{2,1}$. In other words, $\Theta_{1,2}$ and $\Theta_{2,1}$ are the only independent bulk quantities.

For comparison, we now consider three other possibilities for the surface data, which we will refer to as APS- $X$, APS- $Y$, and APS- $Z$ (we explain this terminology below):

$$
\begin{aligned}
& \text { APS- } X: \Omega_{11}=\pi, \Omega_{21}=0, \Omega_{121}=\pi \text {, } \\
& \text { APS- } Y: \Omega_{11}=0, \Omega_{21}=\pi, \Omega_{121}=\pi \text {, } \\
& \text { APS-Z: } \Omega_{11}=0, \Omega_{21}=0, \Omega_{121}=\pi \text {. }
\end{aligned}
$$

Here, as before, we assume that the surface anyons form a group $A=\mathbb{Z}_{2}$ with statistics Eq. (33). Applying the bulkboundary formulas, we can compute $\Theta_{1,2}$ and $\Theta_{2,1}$ in the same way as above. The results are shown in Table I, along with those corresponding to the surface data Eq. (34), which we refer to as CSL.

What conclusions can we draw from these calculations? First, we can see from Table I that $\Theta_{1,2}$ and $\Theta_{2,1}$ take different values in each of the four cases. Therefore, we can conclude that the corresponding bulk spin models all belong to distinct SPT phases. Also, we see that at least one of $\Theta_{1,2}$ and $\Theta_{2,1}$ is nonzero for each of the APS- $X$, APS- $Y$, APS- $Z$ cases listed above, which implies that the corresponding bulk spin models belong to nontrivial SPT phases. Finally, since the bulk data vanish for the CSL case, we can conclude that the corresponding bulk spin model belongs to a trivial SPT phase-if we make the additional assumption that the bulk data are complete.

As we mentioned above, these four types of surfaces were originally discussed by Ref. [20]. Our terminology for these surfaces follows that of Ref. [20]: the reason we refer to the first type of data as CSL is that a variant of the 2D Kalmeyer-Laughlin chiral spin liquid (CSL) state is described by these data; likewise, the reason we refer to the other types of data as APS- $X$, APS- $Y$, and APS- $Z$ is because they correspond to the anomalous projective semion (APS) states of Ref. [20]. Here the word "anomalous" signifies the fact that the last three types of surface

TABLE I. Surface and bulk data for four surfaces with $G=$ $\mathbb{Z}_{2} \times \mathbb{Z}_{2}$ and $A=\mathbb{Z}_{2}$.

\begin{tabular}{lcccccc}
\hline \hline & \multicolumn{3}{c}{ Surface data } & & \multicolumn{2}{c}{ Bulk data } \\
\cline { 2 - 4 } \cline { 7 - 8 } Model & $\Omega_{11}$ & $\Omega_{21}$ & $\Omega_{121}$ & & $\Theta_{1,2}$ & $\Theta_{2,1}$ \\
\hline CSL & $\pi$ & $\pi$ & $\pi$ & & 0 \\
APS- $X$ & $\pi$ & 0 & $\pi$ & & 0 \\
APS- $Y$ & 0 & $\pi$ & $\pi$ & & $\pi$ & 0 \\
APS- $Z$ & 0 & 0 & $\pi$ & & $\pi$ & $\pi$ \\
\hline \hline
\end{tabular}

data are incompatible with a pure 2D lattice model and can exist only on the boundary of a nontrivial 3D SPT phase.

It is worth pointing out that Ref. [20] used a different language to describe the surface data than what we use here. In this alternate description, the symmetry properties of the surface are described by an element $\omega$ of the cohomology group $H^{2}(G, A)$ instead of the quantities $\left\{\Omega_{i \mu}, \Omega_{i j \mu}, x_{i l}^{\mu}\right\}$. We explain this alternative language and its relationship with $\left\{\Omega_{i \mu}, \Omega_{i j \mu}, x_{i l}^{\mu}\right\}$ in Sec. VII.

\section{Example 2}

We can also use the bulk-boundary correspondence in the opposite direction; that is, we can use it to constrain the types of surfaces that are compatible with a given bulk Hamiltonian. For an example of this, imagine that we have a lattice boson model that realizes a SPT phase with symmetry group $G=\mathbb{Z}_{2} \times \mathbb{Z}_{2}$. As we mentioned in the previous section, there are only two independent pieces of bulk data for this symmetry group: $\Theta_{1,2}$ and $\Theta_{2,1}$. Let us suppose that one or both of these quantities takes a nonzero value for our lattice spin model. Using this information we can constrain the possible surfaces of this system. In particular, assuming that the surface anyons are all Abelian and are not permuted by the symmetries, we can show that the group of surface anyons $A=$ $\prod_{\mu=1}^{M} \mathbb{Z}_{N_{\mu}}$ has the property that $N_{\mu}$ is even for at least one value of $\mu$.

One way to see this is to examine the general constraints Eqs. (27)-(29) on $x_{i l}^{\mu}$. In particular, from the constraint Eq. (29), we can see that $x_{11}^{\mu}=x_{22}^{\mu}=0$. Also, if $N_{\mu}$ is odd, then the constraint Eq. (27) implies that $x_{12}^{\mu}=x_{21}^{\mu}=0$. Hence, if $N_{\mu}$ were odd for all $\mu$, then $x_{i l}^{\mu}$ would necessarily vanish completely. But then $\Theta_{1,2}$ and $\Theta_{2,1}$ would also have to vanish according to the bulk-boundary formula Eq. (30). We conclude that if either $\Theta_{1,2}$ or $\Theta_{2,1}$ is nonzero, then at least one of the $N_{\mu}$ 's must be even.

\section{DERIVATION OF THE BULK-BOUNDARY CORRESPONDENCE}

In this section, we derive the bulk-boundary formula Eq. (31) for $\Theta_{i j, l}$. The derivations of the other two formulas [Eqs. (30) and (32)] are similar and are given in Appendixes $\mathrm{C}$ and $\mathrm{D}$.

\section{A. Step 1: Deforming the braiding process}

Our derivation proceeds in four steps. In the first step, we derive an equivalence between the three-loop braiding process associated with $\Theta_{i j, l}$ and another process that involves braiding vortex "arches" on the surface. The key to deriving this equivalence is the general principle that statistical Berry phases are invariant under "smooth" deformations of braiding processes; that is, if two braiding processes can be "smoothly" deformed into one another, 
then the associated statistical Berry phases must be equal. Here, a smooth deformation is a sequence of local changes to the excitations involved in the braiding process. The local changes can be arbitrary except that the moving excitation must stay far apart from the other excitations at every step of the deformation. (Here, when we say "local changes" to the excitations we mean any changes that can be implemented by unitary operators supported in the neighborhood of the excitations).

To begin, let us imagine performing the three-loop braiding process in the bulk [Fig. 5(a)]. That is, we braid a loop $\alpha$ around another loop $\beta$ for $N^{i j}$ times while both are linked to a third loop $\sigma$. Here, $\alpha, \beta, \sigma$ carry unit type- $i$, type$j$, and type- $l$ flux; i.e., $\phi_{\alpha}=\left(2 \pi / N_{i}\right) \mathbf{e}_{i}, \phi_{\beta}=\left(2 \pi / N_{j}\right) \mathbf{e}_{j}$, and $\phi_{\sigma}=\left(2 \pi / N_{l}\right) \mathbf{e}_{l}$. The Berry phase associated with this process is $\Theta_{i j, l}$.

Next, we stretch $\alpha$ and $\beta$ and absorb the bottoms of these loops into the surface [53]. This step changes $\alpha, \beta$ into vortex arches that terminate on the surface [Fig. 5(b)]. After this step, the deformed braiding process involves braiding vortex arches $\alpha, \beta$ while they are both linked to $\sigma$. By the general principle described above, this deformed process must yield the same Berry phase, $\Theta_{i j, l}$.

To proceed further, we now stretch $\sigma$ and absorb its bottom into the surface. This step changes $\sigma$ into another vortex arch [Fig. 5(c)]. Finally, we disentangle the three arches by unwinding one of the two ends of the arch $\sigma$ [Fig. 5(d)]. The braiding process now involves braiding two unlinked arches $\alpha, \beta$ around one another. Again, the Berry phase must be the same as in the original process. Putting this all together, we conclude that $\Theta_{i j, l}$ is equal to the Berry phase associated with braiding the $\operatorname{arch} \alpha$ around the $\operatorname{arch} \beta$ for $N^{i j}$ times, as shown in Fig. 5(d).

At this point, the reader may be puzzled by the following question: given that $\sigma$ is completely decoupled from the other arches, it appears that the Berry phase for the process shown in Fig. 5(d) must be independent of the flux carried by $\sigma$, i.e., independent of $l$. Yet at the same time, we know that this Berry phase is equal to the three-loop braiding statistics $\Theta_{i j, l}$, which certainly depends on $l$. How can this be? The resolution of this paradox is that the vortex arches $\alpha$ and $\beta$ are actually non-Abelian excitations. This means that the Berry phase associated with braiding $\alpha$ and $\beta$ depends on their initial state (and also need not be Abelian, in general). The Berry phase $\Theta_{i j, l}$ occurs for a particular initial state, namely the state obtained through the sequence of deformations shown in Fig. 5. This initial state "knows" about $l$ because unwinding $\sigma$ affects the state of $\alpha$ and $\beta$. We see this explicitly in step 2, below, when we imagine shrinking $\alpha$ and $\beta$ down to the surface.

\section{B. Step 2: Splitting the excitations}

Our task is now to analyze the vortex arch braiding process in Fig. 5(d). Before doing this, it is useful to first consider a thought experiment in which we shrink down the arches $\alpha, \beta$ so that all that is left are two localized surface excitations. From general considerations, we know that the resulting surface excitations can be written as a linear superposition of states, each of which has a definite anyon type. We ask, what types of surface anyons appear in this linear superposition?

The answer is simple: if we shrink $\alpha$, we get a superposition of different surface anyons, all of which have anyonic flux $x_{i l}$; likewise, if we shrink $\beta$, we get a superposition of surface anyons, all of which have anyonic flux $x_{j l}$. To see this, notice that shrinking down $\alpha, \beta, \sigma$ in Fig. 5(d) is very similar to shrinking $\alpha, \sigma$ in Fig. 4. Furthermore, in the latter case, we know that shrinking $\alpha$ gives a superposition of different surface anyons, all of which have anyonic flux $x_{i l}$ [see Eq. (20)]. Therefore, the same must be true for Fig. 5(d).

The most important point from this discussion is that if we shrink $\alpha$ or $\beta$ to the surface, we will generally get nontrivial surface anyons. This property is inconvenient for our subsequent analysis and motivates us to split $\alpha, \beta$ into more "elementary" excitations.

For this reason, the next step in our analysis is to split the arch $\alpha$ into a surface anyon $\mathcal{X}$ and a new arch $\tilde{\alpha}$. Similarly, we split $\beta$ into a surface anyon $\mathcal{Y}$ and a new $\operatorname{arch} \tilde{\beta}$ (Fig. 6). We choose the surface anyons $\mathcal{X}, \mathcal{Y}$ to be any anyons with anyonic flux

$$
\xi_{\mathcal{X}}=x_{i l}, \quad \xi_{\mathcal{Y}}=x_{j l},
$$

while we choose the arches $\tilde{\alpha}, \tilde{\beta}$ to be any arches with the property that $\alpha$ can be written as a fusion product of $\mathcal{X}$ and $\tilde{\alpha}$, and that $\beta$ can be written as a fusion product of $\mathcal{Y}$ and $\tilde{\beta}$. (a)

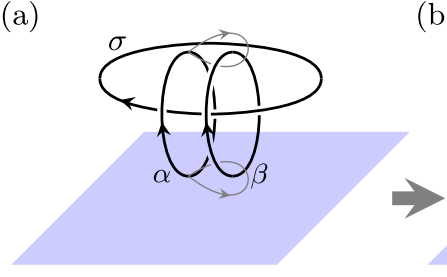

(b)

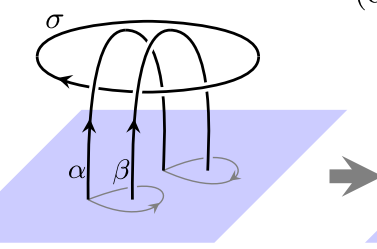

(c)

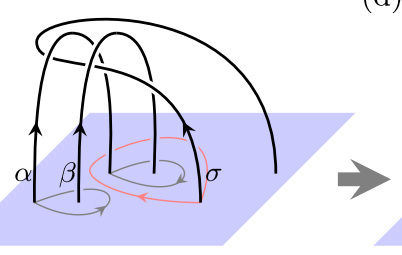

(d)

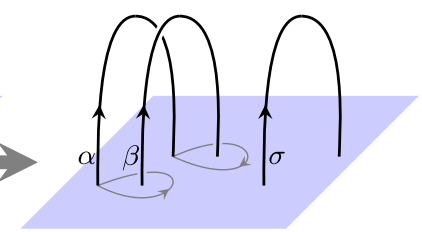

FIG. 5. Deforming a three-loop braiding process (a) in the bulk into a braiding process (d) of vortex arches near the surface. The deformation splits into three steps, (a) $\rightarrow$ (b), (b) $\rightarrow$ (c), and (c) $\rightarrow$ (d). The gray curves in (a)-(d) are trajectories of some points on $\alpha$; the red curve in (c) is the trajectory of one of the end points of $\sigma$. 
The motivation for performing this splitting is that the new arches $\tilde{\alpha}, \tilde{\beta}$ have a nice property, by construction: if we shrink $\tilde{\alpha}, \tilde{\beta}$ to the surface, we get a superposition of charge excitations instead of more complicated surface anyons. This property will play an important role below when we compute the Berry phase associated with braiding $\tilde{\alpha}$ around $\tilde{\beta}$ [see Eq. (59)].

\section{Step 3: Decomposing the braiding process}

After splitting $\alpha, \beta$ as described above, our braiding process reduces to one in which we braid $\tilde{\alpha}$ and $\mathcal{X}$ around $\tilde{\beta}$ and $\mathcal{Y}$ for $N^{i j}$ times (Fig. 6). The next step is to decompose this process into a series of simpler processes.

First, we introduce some notation. Let $|\psi\rangle$ be the initial state at the beginning of the braiding, i.e., the state obtained from the deformation process shown in Fig. 5 followed by the splitting process in Fig. 6 . Let $\mathbb{V}$ be the subspace consisting of all states that are degenerate in energy with $|\psi\rangle$. Here, $\mathbb{V}$ could have dimension 1 or higher. Let $W: \mathbb{V} \rightarrow$ $\mathbb{V}$ be the unitary braiding matrix that describes the (possibly) non-Abelian Berry phase associated with braiding $\tilde{\alpha}$ and $\mathcal{X}$ around $\tilde{\beta}$ and $\mathcal{Y}$ once.

Then, with this notation, the fact that the Berry phase for our braiding process is $\Theta_{i j, l}$ translates to the equation

$$
W^{N^{i j}}|\psi\rangle=e^{i \Theta_{i j, l}}|\psi\rangle
$$

To proceed further, it is helpful to represent this braiding process using a $2 \mathrm{D}$ picture. One way to do this is to imagine folding the surface and straightening the vortex arches, as shown in Fig. 7. After doing this, the braiding process now involves vertical vortex lines. If we now take a top view of

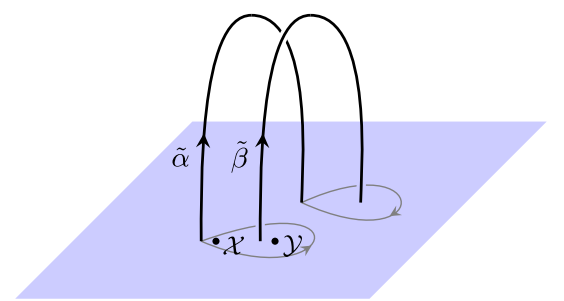

FIG. 6. Splitting $\alpha$ into $\tilde{\alpha}$ and $\mathcal{X}$, and splitting $\beta$ into $\tilde{\beta}$ and $\mathcal{Y}$.

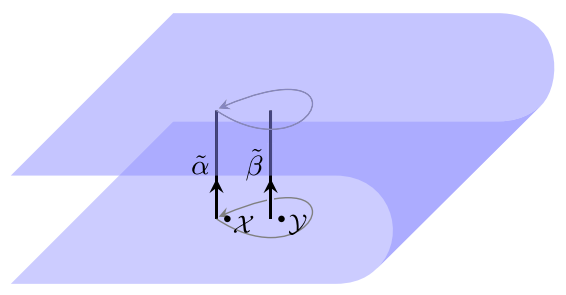

FIG. 7. Folding the surface and straightening the vortex lines in Fig. 6. In between the top and bottom parts of the surface is the gauged SPT model, and outside is the vacuum.
Fig. 7, then $W$ can be visualized as



With this picture in mind, it is easy to see how to decompose the above braiding process into simpler processes. In particular, let $W_{\tilde{\alpha} \tilde{\beta}}, W_{\tilde{\alpha} \mathcal{Y}}, W_{\mathcal{X} \tilde{\beta}}$, and $W_{\mathcal{X Y}}$ be the braid matrices corresponding to the following processes:
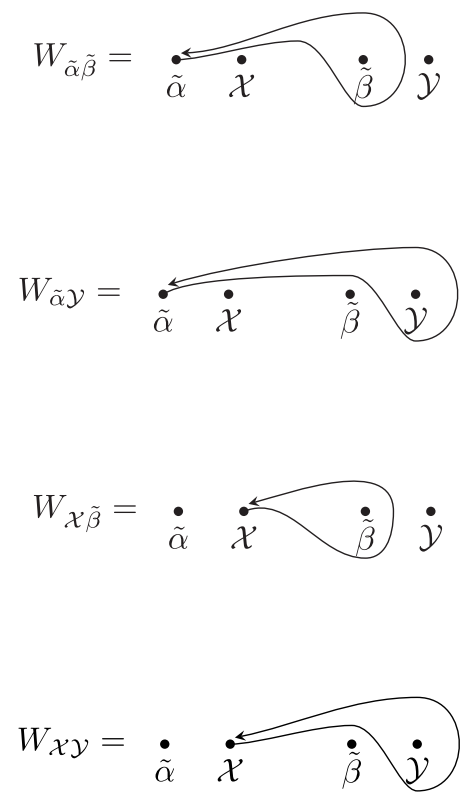

Then, it is easy to see that if we perform the above four processes sequentially, the result can be smoothly deformed into the process corresponding to $W$. Translating this into algebra, we derive

$$
W=W_{\mathcal{X Y}} W_{\mathcal{X} \tilde{\beta}} W_{\tilde{\alpha} \mathcal{Y}} W_{\tilde{\alpha} \tilde{\beta}}
$$

Substituting this into Eq. (38), we obtain

$$
\left(W_{\mathcal{X Y}} W_{\mathcal{X} \tilde{\beta}} W_{\tilde{\alpha} \mathcal{Y}} W_{\tilde{\alpha} \tilde{\beta}}\right)^{N_{i j}}|\psi\rangle=e^{i \Theta_{i j, l}}|\psi\rangle
$$

Equation (45) is the main result of this step.

\section{Step 4: Evaluating Eq. (45)}

We now evaluate the expression on the left-hand side of Eq. (45). We accomplish this using several algebraic properties of the braid matrices $W_{\mathcal{X Y}}, W_{\mathcal{X} \tilde{\beta}}, W_{\tilde{\alpha} \mathcal{Y}}$, and $W_{\tilde{\alpha} \tilde{\beta}}$ that we derive below. The first property is that these braid matrices all commute with each other, except for the pairs $\left(W_{\tilde{\alpha} \tilde{\beta}}, W_{\mathcal{X} \tilde{\beta}}\right)$ and $\left(W_{\tilde{\alpha} \tilde{\beta}}, W_{\tilde{\alpha} \mathcal{y}}\right)$, which obey the commutation relations 


$$
\begin{aligned}
& W_{\tilde{\alpha} \tilde{\beta}} W_{\mathcal{X} \tilde{\beta}}=e^{i \zeta_{1}} \times W_{\mathcal{X} \tilde{\beta}} W_{\tilde{\alpha} \tilde{\beta}}, \\
& W_{\tilde{\alpha} \tilde{\beta}} W_{\tilde{\alpha} \mathcal{Y}}=e^{i \zeta_{2}} \times W_{\tilde{\alpha} \mathcal{Y}} W_{\tilde{\alpha} \tilde{\beta}},
\end{aligned}
$$

where $\zeta_{1}, \zeta_{2}$ are given by

$$
\zeta_{1}=\sum_{\mu} x_{i l}^{\mu} \Omega_{i j \mu}, \quad \zeta_{2}=\sum_{\mu} x_{j l}^{\mu} \Omega_{j i \mu} .
$$

Another important property of these matrices is that when we raise them to the power $N^{i j}$, they simplify considerably:

$$
\begin{gathered}
W_{\mathcal{X} \tilde{\beta}}^{N^{i j}}=e^{i \zeta_{3}} \hat{I}, \\
W_{\tilde{\alpha} \mathcal{Y}}^{N^{i j}}=e^{i \zeta_{4}} \hat{I}, \\
W_{\mathcal{X Y}}^{N^{i j}}=\hat{I}, \\
W_{\tilde{\alpha} \tilde{\beta}}^{N^{i j}}|\psi\rangle=|\psi\rangle,
\end{gathered}
$$

where $\hat{I}$ is the identity matrix in $\mathbb{V}$, and $\zeta_{3}, \zeta_{4}$ are given by

$$
\zeta_{3}=\sum_{\mu} \frac{N^{i j}}{N^{j \mu}} x_{i l}^{\mu} \Omega_{j \mu}, \quad \zeta_{4}=\sum_{\mu} \frac{N^{i j}}{N^{i \mu}} x_{j l}^{\mu} \Omega_{i \mu} .
$$

Notice that the last relation [Eq. (51)] is different from the others because it tells us only about the action of a braid matrix on the specific state $|\psi\rangle$ defined above. In contrast, the other relations are matrix equations that hold throughout the degenerate subspace $\mathbb{V}$.

We now use these relations to evaluate the left-hand side of Eq. (45) and thereby derive the bulk-boundary formula Eq. (31). First, from the commutation relations Eqs. (46) and (47), we reorder operators at the cost of a phase factor:

$$
\begin{aligned}
\left(W_{\mathcal{X Y}} W_{\mathcal{X} \tilde{\beta}} W_{\tilde{\alpha} \mathcal{Y}} W_{\tilde{\alpha} \tilde{\beta}}\right)^{N^{i j}}|\psi\rangle= & W_{\mathcal{X} \mathcal{Y}}^{N^{i j}} W_{\mathcal{X} \tilde{\beta}}^{N^{i j}} W_{\tilde{\alpha} \mathcal{Y}}^{N^{i j}} W_{\tilde{\alpha} \tilde{\beta}}^{N^{i j}}|\psi\rangle \\
& \times e^{\left[i N^{i j}\left(N^{i j}-1\right) / 2\right]\left(\zeta_{1}+\zeta_{2}\right)}
\end{aligned}
$$

Here, the phase factor comes from the fact that the commutation relations Eqs. (46) and (47) are used $\left(N^{i j} / 2\right)\left(N^{i j}-1\right)$ times during reordering.

Next, we apply the identities Eqs. (48)-(51) to obtain

$$
W_{\mathcal{X} \mathcal{Y}}^{N^{i j}} W_{\mathcal{X} \tilde{\beta}}^{N^{i j}} W_{\tilde{\alpha} \mathcal{Y}}^{N^{i j}} W_{\tilde{\alpha} \tilde{\beta}}^{N^{i j}}|\psi\rangle=e^{i\left(\zeta_{3}+\zeta_{4}\right)}|\psi\rangle .
$$

Putting this together, we derive

$$
\begin{aligned}
\left(W_{\mathcal{X Y}} W_{\mathcal{X} \tilde{\beta}} W_{\tilde{\alpha} \mathcal{Y}} W_{\tilde{\alpha} \tilde{\beta}}\right)^{N^{i j}}|\psi\rangle= & e^{i\left(\zeta_{3}+\zeta_{4}\right)}|\psi\rangle \\
& \times e^{i\left[N^{i j}\left(N^{i j}-1\right) / 2\right]\left(\zeta_{1}+\zeta_{2}\right)} .
\end{aligned}
$$

Comparing with Eq. (45), we conclude that

$$
\Theta_{i j, l}=\zeta_{3}+\zeta_{4}+\frac{N^{i j}\left(N^{i j}-1\right)}{2}\left(\zeta_{1}+\zeta_{2}\right)
$$

This is nothing other than the bulk-boundary formula Eq. (31), as one can see from the definitions of $\zeta_{1}, \zeta_{2}$, $\zeta_{3}, \zeta_{4}$.

To complete the argument, we now derive the algebraic relations Eqs. (46)-(51). We begin by proving the statement that precedes Eq. (46), namely, that the braid matrices all commute with each other except for the pairs $\left(W_{\tilde{\alpha} \tilde{\beta}}, W_{\mathcal{X} \tilde{\beta}}\right)$ and $\left(W_{\tilde{\alpha} \tilde{\beta}}, W_{\tilde{\alpha} \mathcal{y}}\right)$. To establish this statement, it suffices to show that (1) $W_{\mathcal{X Y}}$ commutes with the other three operators and that (2) $W_{\tilde{\alpha} y}$ and $W_{\mathcal{X} \tilde{\beta}}$ commute with one another. The first result follows from the fact that the mutual statistics between surface anyons is Abelian, so that $W_{\mathcal{X Y}}$ is proportional to the identity $\hat{I}$. The second result follows from the observation that the braiding paths associated with $W_{\tilde{\alpha} \mathcal{Y}}$ and $W_{\mathcal{X} \tilde{\beta}}$ do not overlap.

Next, we need to establish the commutation relations Eqs. (46) and (47). Proving these relations is more technical and, hence, we postpone their derivation to Appendix E. Here, we give only the intuitive picture behind these relations. Consider for example Eq. (46). This relation can be equivalently written as

$$
e^{i \zeta_{1} \hat{I}}=W_{\tilde{\alpha} \tilde{\beta}}^{-1} W_{\mathcal{X} \tilde{\beta}}^{-1} W_{\tilde{\alpha} \tilde{\beta}} W_{\mathcal{X} \tilde{\beta}}
$$

To understand the meaning of the right-hand side, remember that braiding processes are symmetrical in the sense that braiding $X$ around $Y$ is topologically equivalent to braiding $Y$ around $X$ (for properly chosen paths). Therefore, the product on the right-hand side can be interpreted as a process in which $\tilde{\beta}$ is first braided around $\mathcal{X}$, then around $\tilde{\alpha}$, then around $\mathcal{X}$ in the opposite direction, and finally around $\tilde{\alpha}$ in the opposite direction. This is very similar to the braiding process that defines $\Omega_{i j \mu}$, except for two differences: (1) the moving excitation is the vortex $\tilde{\beta}$ rather than the anyon $\mathcal{X}$, and (2) the anyon $\mathcal{X}$ carries anyonic flux $\xi_{\mathcal{X}}=x_{i l}$ instead of having unit type- $\mu$ flux. It turns out that the first difference is irrelevant and can be safely ignored. On the other hand, the second difference is important and changes the product on the left-hand side from $e^{i \Omega_{i j \mu} \hat{I}}$ to $e^{i \sum_{\mu} x_{i l}^{\mu} \Omega_{i j \mu}} \hat{I}$ (see Appendix E).

We now move on to prove the identities Eqs. (48)-(51). We begin with Eq. (50) since it is the simplest to derive. To prove this identity, recall that the surface anyons $\mathcal{X}, \mathcal{Y}$ have Abelian statistics so the braid matrix $W_{\mathcal{X Y}}$ takes the form

$$
W_{\mathcal{X Y}}=e^{i \theta_{\mathcal{X}} \hat{I}}
$$


Next, from Eqs. (14) and (13) we see that

$$
\theta_{\mathcal{X Y}}=\theta_{\xi_{\mathcal{X} \xi \mathcal{Y}}}=\sum_{\mu \nu} x_{i l}^{\mu} x_{j l}^{\nu} \Phi_{\mu \nu}
$$

where we use $\xi_{\mathcal{X}}=x_{i l}$ and $\xi_{\mathcal{Y}}=x_{j l}$. Combining these two results, we obtain the identity

$$
W_{\mathcal{X Y}}=e^{i \sum_{\mu \nu} u_{i l}^{\mu} x_{j l}^{\nu} \Phi_{\mu \nu}} \hat{I},
$$

If we now raise both sides to the $N^{i j}$ power, we can see that the right-hand side reduces to the identity operator $\hat{I}$ since $N^{i j} x_{i l}^{\mu}$ is a multiple of $N_{\mu}$ according to Eq. (27), and $N_{\mu} \Phi_{\mu \nu}$ is a multiple of $2 \pi$ according to Eq. (21). We conclude that $W_{\mathcal{X Y}}^{N^{i j}}=\hat{I}$ as claimed.

Next, we consider Eq. (51). To derive this result, we use a property of $|\psi\rangle$ that we discuss in Sec. V B: if we shrink $\tilde{\alpha}$ or $\tilde{\beta}$ down to the surface, the result is a superposition of charge excitations. To see why this property is useful, let us consider the special case where shrinking $\tilde{\alpha}, \tilde{\beta}$ gives definite charge excitations $q_{\tilde{\alpha}}, q_{\tilde{\beta}}$ instead of a superposition of different charges. In this case, it is easy to see that the statistical phase $\theta$ associated with braiding $\tilde{\alpha}$ around $\tilde{\beta}$ is given by the Aharonov-Bohm law: $\theta=q_{\tilde{\alpha}} \cdot \phi_{\tilde{\beta}}+q_{\tilde{\beta}} \cdot \phi_{\tilde{\alpha}}$. Here, the first term comes from braiding the charge $q_{\tilde{\alpha}}$ around the flux $\phi_{\tilde{\beta}}$, while the second term comes from braiding the flux $\phi_{\tilde{\alpha}}$ around the charge $q_{\tilde{\beta}}$. (See Ref. [40] for a derivation of this formula in a closely related context.) Translating this equation into our algebraic notation gives

$$
W_{\tilde{\alpha} \tilde{\beta}}|\psi\rangle=e^{i\left(q_{\tilde{\alpha}} \cdot \phi_{\tilde{\beta}}+q_{\tilde{\beta}} \cdot \phi_{\tilde{\alpha}}\right)}|\psi\rangle .
$$

Now recall that $\tilde{\alpha}$ is a unit type- $i$ flux while $\tilde{\beta}$ is a unit type- $j$ flux, so that $\phi_{\tilde{\alpha}}=\left(2 \pi / N_{i}\right) \mathbf{e}_{i}$ and $\phi_{\tilde{\beta}}=\left(2 \pi / N_{j}\right) \mathbf{e}_{j}$. Substituting this into the above equation, and raising both sides to the $N^{i j}$ power, we see that $W_{\tilde{\alpha} \tilde{\beta}}^{N^{i j}}|\psi\rangle=|\psi\rangle$. This establishes Eq. (51) for the special case where shrinking $\tilde{\alpha}$, $\tilde{\beta}$ gives definite charge excitations. In fact, since the equation $W_{\tilde{\alpha} \tilde{\beta}}^{N^{i j}}|\psi\rangle=|\psi\rangle$ holds independent of the values of $q_{\tilde{\alpha}}, q_{\tilde{\beta}}$, the superposition principle implies that it must also hold in the case where shrinking $\tilde{\alpha}, \tilde{\beta}$ gives a superposition of charge excitations. This proves Eq. (51) in the general case.

Finally, we need to discuss Eqs. (48) and (49). The proof of these relations is technical, so we postpone it to Appendix E. However, the physical picture for these relations is simple. For example, consider Eq. (48). We can see that $\zeta_{3}$ is the Berry phase associated with braiding $\mathcal{X}$ around $\tilde{\beta}$ for $N^{i j}$ times. If we compare this braiding process to the one that defines $\Omega_{j \mu}$, we see that they are very similar, except for two differences: (1) $\mathcal{X}$ carries anyonic flux $\xi_{\mathcal{X}}=x_{i l}$ instead of carrying unit type- $\mu$ flux, and (2) $\mathcal{X}$ is braided $N^{i j}$ times instead of $N^{j \mu}$ times. Given these two differences, it is perhaps not surprising that the Berry phase for this process is $\sum_{\mu}\left(N^{i j} / N^{j \mu}\right) x_{i l}^{\mu} \Omega_{j \mu}$ instead of $\Omega_{j \mu}$.

\section{IMPLICATIONS FOR PURELY 2D SYSTEMS}

Thus far, we have used the data $\left\{\Phi_{\mu}, \Phi_{\mu \nu}, \Omega_{i \mu}, \Omega_{i j \mu}, x_{i l}^{\mu}\right\}$ to describe surfaces of 3D systems. However, the same data can also be used to describe purely $2 D$ systems. More specifically, suppose we are given a $2 \mathrm{D}$ gapped lattice boson model with Abelian symmetry group $G=\prod_{i=1}^{K} \mathbb{Z}_{N_{i}}$ and Abelian anyon excitations described by a group $A=\prod_{\mu=1}^{M} \mathbb{Z}_{N_{\mu}}$. Suppose, in addition, that the symmetry action does not permute the anyons. Then we can define the quantities $\left\{\Phi_{\mu}, \Phi_{\mu \nu}, \Omega_{i \mu}, \Omega_{i j \mu}, x_{i l}^{\mu}\right\}$ for this $2 \mathrm{D}$ system in the same way as we did for surfaces-with $\Phi_{\mu}, \Phi_{\mu \nu}$ describing the exchange and mutual statistics of anyons, $\Omega_{i \mu}, \Omega_{i j \mu}$ describing the braiding statistics between anyons and vortices, and $x_{i l}^{\mu}$ given by Eq. (18).

Now that we have defined $\left\{\Phi_{\mu}, \Phi_{\mu \nu}, \Omega_{i \mu}, \Omega_{i j \mu}, x_{i l}^{\mu}\right\}$ for 2D systems, we can ask what happens if we insert this data into the right-hand side of Eqs. (30)-(32) and compute the corresponding "3D bulk" quantities $\Theta_{i, l}, \Theta_{i j, l}, \Theta_{i j k, l}$. In this section, we argue that if we do this, then these bulk quantities will always vanish. That is, for any $2 \mathrm{D}$ system we have

$$
\Theta_{i, l}=\Theta_{i j, l}=\Theta_{i j k, l}=0,
$$

where $\Theta_{i, l}, \Theta_{i j, l}, \Theta_{i j k, l}$ are defined by Eqs. (30)-(32). We can think of the above equations as constraints on which data $\left\{\Phi_{\mu}, \Phi_{\mu \nu}, \Omega_{i \mu}, \Omega_{i j \mu}, x_{i l}^{\mu}\right\}$ can be realized by $2 \mathrm{D}$ systems.

The simplest way to derive the constraints Eq. (60) is to think of our $2 \mathrm{D}$ system as living on the surface of the $3 \mathrm{D}$ vacuum. It is clear that $\Theta_{i, l}, \Theta_{i j, l}, \Theta_{i j k, l}$ vanish for the 3D vacuum, so by using the bulk-boundary formulas, Eqs. (30)-(32), we obtain three constraints on $\left\{\Phi_{\mu}, \Phi_{\mu \nu}, \Omega_{i \mu}, \Omega_{i j \mu}, x_{i l}^{\mu}\right\}$, which are precisely Eq. (60).

While the above argument is perfectly solid, it is instructive to rederive the constraints using purely twodimensional arguments. In what follows, we present such an argument for one of the three constraints, namely, $\Theta_{i j, l}=0$. (Similar arguments can be used to establish the other constraints.) At the heart of our derivation is a particular braiding process that we describe below. Our strategy is to compute the statistical phase associated with this process in two ways: in one approach we see that the statistical phase is given by the right-hand side of Eq. (31), while in the other approach we see that the phase vanishes. Combining the two calculations, we then derive $\Theta_{i j, l}=0$.

Before describing the braiding process, we first need to describe the initial state $|\Psi\rangle$ at the beginning of the process. Imagine that we start in the ground state. We then create three vortex-antivortex pairs, $\{\alpha, \bar{\alpha}\},\{\beta, \bar{\beta}\}$, and $\{\sigma, \bar{\sigma}\}$, where $\alpha, \beta, \sigma$ carry unit type- $i$, type- $j$, and type- $l$ gauge flux [Fig. 8(a)]. We then braid $\sigma$ around $\bar{\alpha}$ and $\bar{\beta}$ [Fig. 8(b)]. 
(a)



(b)

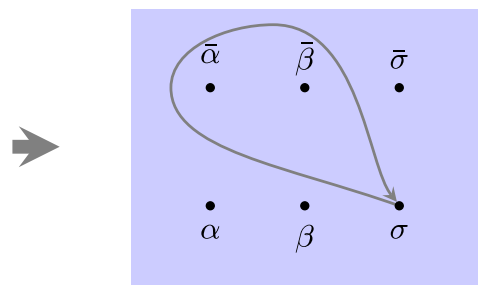

(c)



FIG. 8. A 2D braiding process that shows that $\Theta_{i j, l}$ vanishes for strictly 2D systems. See the main text for details.

The state obtained in this way is the initial state $|\Psi\rangle$ for our braiding. The braiding process itself is rather simple: starting in the state $|\Psi\rangle$, we braid $\alpha$ around $\beta$ for $N^{i j}$ times in the counterclockwise direction, while simultaneously braiding $\bar{\alpha}$ around $\bar{\beta}$ for $N^{i j}$ times in the clockwise direction [Fig. 8(c)].

Let us try to compute the statistical phase associated with this process. To this end, notice that there is a close analogy between the 2D braiding process shown in Fig. 8(c) and the 3D arch braiding process shown in Fig. 5(d): the 2D braiding process looks like a horizontal cross section of the 3D process. There is also a close connection between the initial state $|\Psi\rangle$ for the 2D process and the initial state $|\psi\rangle$ for the $3 \mathrm{D}$ process since the two states are obtained from similar manipulations of vortices [compare Figs. 8(a) and 8(b) with Figs. 5(a)-5(c)]. Because of these similarities, we can compute the statistical phase for the 2D process using essentially the same calculation as in the 3D case discussed in Sec. V. In the first step, we split the vortex $\alpha$ into another vortex $\tilde{\alpha}$ together with an anyon $\mathcal{X}$ carrying anyonic flux $\xi_{\mathcal{X}}=x_{i l}$. Also, we split $\beta$ into $\tilde{\beta}$ and $\mathcal{Y}$, where $\xi_{y}=x_{j l}$. Then, after performing the splitting, we decompose the braiding process shown in Fig. 8(c) into simpler processes involving $\mathcal{X}, \mathcal{Y}, \tilde{\alpha}, \tilde{\beta}$, etc. Using the same arguments as in Sec. V, we can express the statistical phases for these simpler processes in terms of $\left\{\Phi_{\mu}, \Phi_{\mu \nu}, \Omega_{i \mu}, \Omega_{i j \mu}, x_{i l}^{\mu}\right\}$ and then put everything together to obtain the statistical phase for the whole process. Since the computation is almost identical to the $3 \mathrm{D}$ case, the result is also the same; that is, one finds that the statistical phase is given by the right-hand side of Eq. (31).

Now we compute the statistical phase using a different approach and show that it vanishes. This alternate approach is based on the observation that the two braiding processes shown in Figs. 8(b) and 8(c) commute with one another, since they do not overlap. This commutativity means that instead of starting our braiding process [Fig. 8(c)] in the state $|\Psi\rangle$, which is obtained after we do the braiding in Fig. 8(b), we can equally well start our braiding process in the state $\left|\Psi^{\prime}\right\rangle$, which is obtained before we do the braiding in Fig. 8(b). But if we start in the state $\left|\Psi^{\prime}\right\rangle$, then it is easy to see that the statistical phase for our braiding process must vanish. In fact, even a single braiding of $\alpha$ around $\beta$ in the counterclockwise direction together with a simultaneous braiding of $\bar{\alpha}$ around $\bar{\beta}$ in the clockwise direction already gives a vanishing statistical phase [Fig. 9(a)]. To see this, note that in the state $\left|\Psi^{\prime}\right\rangle$, the two pairs $\alpha, \bar{\alpha}$ and $\beta, \bar{\beta}$ are both in the vacuum fusion channel. This means that we can annihilate $\alpha$ and $\bar{\alpha}$ with local operators, if we bring them close together. Similarly, we can annihilate $\beta$ and $\bar{\beta}$. Using this fact, we can deform the braiding process shown in Fig. 9(a) so that we annihilate $\alpha$ and $\bar{\alpha}$ at some stage of the braiding and recreate them at a later stage [Fig. 9(b)]. After this, we can further annihilate the pair $\beta, \bar{\beta}$ [Fig. 9(c)]. Finally, we can deform the process so that $\alpha$ and $\bar{\alpha}$ are braided around the vacuum [Fig. 9(d)]. Clearly, the statistical phase associated with Fig. 9(d) is zero, so since the deformation cannot change the statistical phase, we conclude that the statistical phase for the original braiding process shown in Fig. 9(a) must also vanish. Comparing this calculation with the previous one, we conclude that $\Theta_{i j, l}=0$. (a)

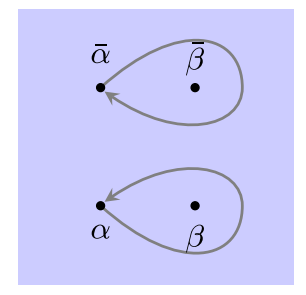

(d)

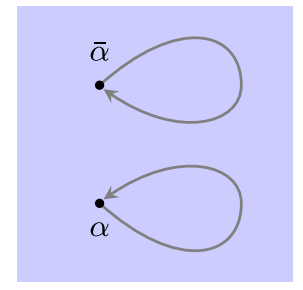

(b)

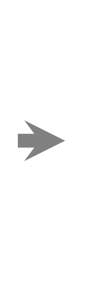

(c)

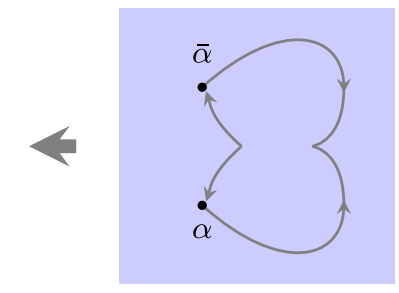

FIG. 9. Deforming a process in which $\alpha$ is braided around $\beta$ and simultaneously $\bar{\alpha}$ is braided around $\bar{\beta}$ in the opposite direction. In the initial state $\left|\Psi^{\prime}\right\rangle$ before the braiding starts, the two pairs $\alpha, \bar{\alpha}$ and $\beta, \bar{\beta}$ are both in the vacuum fusion channel. See the main text for details. 


\section{CONNECTION WITH GROUP COHOMOLOGY}

Recall that the group cohomology models are exactly soluble lattice models that can realize SPT phases in arbitrary spatial dimension [7]. One needs to specify two pieces of information to construct a $d$-dimensional group cohomology model: (1) a symmetry group $G$ and (2) a $(d+1)$-cocycle $\nu$, i.e., a function $\nu: G^{d+1} \rightarrow \mathrm{U}(1)$ satisfying certain algebraic properties. It can be shown that if two cocycles $\nu_{1}$ and $\nu_{2}$ differ by a $(d+1)$-coboundary $\chi$, that is, $\nu_{1}=\nu_{2} \chi$, then the corresponding models are identical to one another. Thus, the distinct group cohomology models are parametrized by elements of the cohomology group $H^{d+1}(G, \mathrm{U}(1))$.

Focusing on the 3D case, the group cohomology models raise a basic question: Which surfaces can exist on the boundary of a 3D group cohomology model with 4-cocycle $\nu$ ?

Chen et al. [20] proposed a possible answer to this question for surfaces that are (1) gapped and symmetric and (2) have the property that the symmetry does not permute the surface anyons. Specifically, Chen et al. conjectured that any surface of this kind must obey the relation

$$
\begin{aligned}
& \nu(a, b, c, d) \\
& \sim R_{\omega(c, d), \omega(a, b)} \\
& \times F_{\omega(b, c), \omega(a, b+c), \omega(a+b+c, d)} F_{\omega(b, c), \omega(b+c, d), \omega(a, b+c+d)}^{-1} \\
& \times F_{\omega(a, b), \omega(c, d), \omega(a+b, c+d)} F_{\omega(a, b), \omega(a+b, c), \omega(a+b+c, d)}^{-1} \\
& \times F_{\omega(c, d), \omega(b, c+d), \omega(a, b+c+d)} F_{\omega(c, d), \omega(a, b), \omega(a+b, c+d)}^{-1},
\end{aligned}
$$

where $a, b, c, d \in G$ and $R_{x y}, F_{x y z}$, and $\omega: G^{2} \rightarrow A$ are various pieces of data that describe the properties of the surface. This conjecture was motivated by the authors' analysis of anomalies in 2D anyon systems.

We make two comments about the notation in Eq. (61). First, the " $\sim$ " sign means that the left- and right-hand sides are equal up to multiplication by a 4-coboundary $\chi(a, b, c, d)$. Second, we use the " + " symbol for the group law because we assume that $G$ is Abelian in what follows.

It is interesting to compare Eq. (61) to the predictions of the bulk-boundary formulas [Eqs. (30)-(32)]. Indeed, for each group cohomology model we can compute the corresponding bulk data $\Theta_{i, l}, \Theta_{i j, l}, \Theta_{i j k, l}$. If we substitute this bulk data into the bulk-boundary formulas Eqs. (30)(32), we can obtain constraints on the surface data $\left\{\Phi_{\mu}, \Phi_{\mu \nu}, \Omega_{i \mu}, \Omega_{i j \mu}, x_{i l}^{\mu}\right\}$, as illustrated by the example in Sec. IV C. Since both the bulk-boundary formulas Eqs. (30)-(32) and (61) give constraints on the set of allowed surfaces, we can ask how these constraints are related to one another. In this section, we show that these constraints are exactly equivalent.
We establish this equivalence in several steps. First, in Sec. VII A, we review the definition of the surface data $\left\{R_{x y}, F_{x y z}, \omega\right\}$. Next, in Sec. VII B, we show how to translate between the two types of surface data, that is, $\left\{R_{x y}, F_{x y z}, \omega\right\}$ and $\left\{\Phi_{\mu}, \Phi_{\mu \nu}, \Omega_{i \mu}, \Omega_{i j \mu}, x_{i l}^{\mu}\right\}$. Similarly, in Sec. VII C, we review how to compute the bulk data $\left\{\Theta_{i, l}, \Theta_{i j, l}, \Theta_{i j k, l}\right\}$ corresponding to a 4-cocycle $\nu$. Finally, in Sec. VII D, we put everything together and derive the equivalence between Eq. (61) and the constraints coming from the bulk-boundary formulas Eqs. (30)-(32). See Fig. 10 for a summary of these results.

\section{A. Review of surface data $\left\{\boldsymbol{R}_{x y}, \boldsymbol{F}_{x y z}, \boldsymbol{\omega}\right\}$}

We begin by reviewing the definition of $\left\{R_{x y}, F_{x y z}, \omega\right\}$. Unlike $\left\{\Phi_{\mu}, \Phi_{\mu \nu}, \Omega_{i \mu}, \Omega_{i j \mu}, x_{i l}^{\mu}\right\}$, these quantities are all defined using the ungauged lattice boson models. The first two quantities, $R_{x y}$ and $F_{x y z}$, are relatively easy to explain. These are the " $R$ symbols" and " $F$ symbols" [47] that describe the braiding and associativity relations of the Abelian anyons that live on the surface. These quantities take values in $\mathrm{U}(1)$, while their indices $x, y, z$ run over the group $A$ of Abelian surface anyons. (Here, we suppress extra indices that are often included in these symbols, e.g., $R_{x y}^{z}$, since these indices are redundant in the Abelian case.)

The other piece of data, $\omega$, is an element of the cohomology group $H^{2}(G, A)$. More concretely, $\omega: G \times$ $G \rightarrow A$ is a function that obeys the relation

$$
\omega(a, b)+\omega(a+b, c)=\omega(b, c)+\omega(a, b+c)
$$

and is defined up to the gauge transformation

$$
\omega(a, b) \rightarrow \omega(a, b)+\chi(a+b)-\chi(a)-\chi(b),
$$

where $\chi: G \rightarrow A$ is some arbitrary function. Here, $\omega(a, b)+\omega(a+b, c)$ denotes the fusion product of the Abelian anyons $\omega(a, b)$ and $\omega(a+b, c)$, while $a+b$ denotes the group composition of $a, b \in G$.

The physical meaning of $\omega$ is that it describes how the symmetry acts on the surface anyons. (See Refs. [36,37,47,51] for general discussions about symmetry actions on anyons.) To explain the precise definition, let us first consider the simpler case of a purely $2 \mathrm{D}$ anyon system (as opposed to a surface). In the purely 2D case, $\omega$ is defined as follows. For each group element $a \in G$, we can construct a corresponding "defect line" by twisting the Hamiltonian along some line running from some point $r_{0}$ to infinity. Here, by "twisting" the Hamiltonian we mean that we conjugate the Hamiltonian by a global symmetry transformation $S_{a}$ acting on one side of the defect line: $H_{\mathrm{tw}}=S_{a}^{-1} H S_{a}$. Importantly, there is some ambiguity in defining this twisting procedure near the end of the defect line, $r_{0}$. Because of this ambiguity, we can construct many different defect line Hamiltonians $H_{\mathrm{tw}}, H_{\mathrm{tw}}^{\prime}, \ldots$ for the 


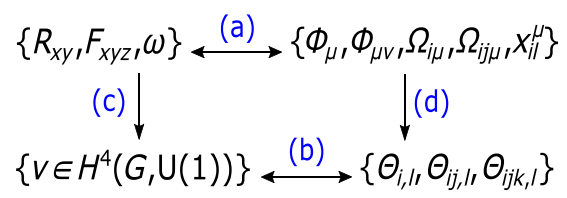

FIG. 10. The equivalence between Eq. (61) and the bulkboundary formulas, Eqs. (30)-(32). The arrow (a) represents the one-to-one mapping between the surface data used in Eq. (61) and in Eqs. (30)-(32). The arrow (b) represents the one-to-one mapping between the bulk data used in Eq. (61) and in Eqs. (30)(32). Arrows (c) and (d) represent Eq. (61) and Eqs. (30)-(32), respectively. We show that the whole diagram commutes in Sec. VII D.

same group element $a \in G$. These Hamiltonians are all on an equal footing, so their ground states are equally good definitions of defect lines. At the same time, if we compare the ground states of different Hamiltonians, $H_{\mathrm{tw}}, H_{\mathrm{tw}}^{\prime}$, they can differ in general by the attachment of an anyon $x \in A$ at the end of the defect line.

Now choose a representative defect line for each $a \in G$. Consider two defect lines corresponding to $a, b \in G$. If we "fuse" the two lines together, we get a defect line corresponding to $a+b$ (Fig. 11). In general, this defect line will differ from our representative $a+b$ defect line by an anyon $x \in A$, as discussed above. We then define a function $\omega: G \times G \rightarrow A$ by $\omega(a, b)=x$. Note that $\omega(a, b) \neq \omega(b, a)$ in general because, unlike point particles, the fusion of defect lines need not be commutative; i.e., there is a well-defined distinction between the defect line on the left and the defect line on the right.

It is not hard to see that this function $\omega$ obeys the constraint Eq. (62) and is well defined up to the transformation Eq. (63). Indeed, to derive the constraint Eq. (62), consider three defect lines $a, b, c$, and imagine fusing them together in two different ways. Using the fact that the two different fusion outcomes must be consistent with one another, one can see that the function $\omega$ obeys the constraint Eq. (62). As for the gauge transformation Eq. (63), this follows from the fact that we can freely change our choice of representatives for each defect line $a$.

Thus far, we have defined $\omega$ for purely 2D systems. We still need to explain how to define $\omega$ for a surface. In this case, we can use essentially the same definition as before (a)

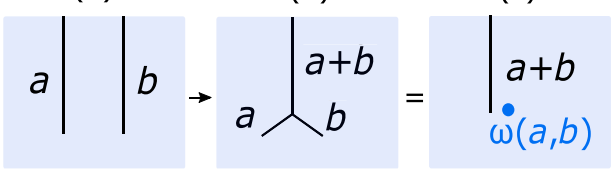

FIG. 11. Definition of $\omega(a, b)$. Panel (a) shows two defect lines labeled by $a$ and $b$. In (b), we fuse the two defect lines and get a defect line corresponding to $a+b$. This defect line differs from the representative $a+b$ defect line shown in (c) by an anyon $\omega(a, b)$. but with one extra dimension; in particular, instead of twisting the Hamiltonian along a defect line that ends at a point, we need to twist it along a defect plane that ends at a line that is perpendicular to the surface. The rest of the definition follows the 2D case in the obvious way [54].

\section{B. Translating between the two types of surface data}

Now, we explain how to translate between the two types of surface data:

$$
\left\{R_{x y}, F_{x y z}, \omega\right\} \leftrightarrow\left\{\Phi_{\mu}, \Phi_{\mu \nu}, \Omega_{i \mu}, \Omega_{i j \mu}, x_{i l}^{\mu}\right\} .
$$

This translation problem can be divided into two pieces, one of which involves the braiding statistics data, namely, $\left\{R_{x y}, F_{x y z}\right\}$ and $\left\{\Phi_{\mu}, \Phi_{\mu \nu}\right\}$, and the other of which involves the symmetry data, namely, $\omega$ and $\left\{\Omega_{i \mu}, \Omega_{i j \mu}, x_{i l}^{\mu}\right\}$.

First, we fix our notation. As in the previous sections, we assume that the symmetry group is $G=\prod_{i=1}^{K} \mathbb{Z}_{N_{i}}$ while the anyon group is $A=\prod_{\mu=1}^{M} \mathbb{Z}_{N_{\mu}}$. We parametrize group elements $a \in G$ by $K$-component integer vectors $a=\left(a_{1}, \ldots, a_{K}\right)$, while we parametrize anyons $x \in A$ by $M$-component integer vectors $x=\left(x^{1}, \ldots, x^{M}\right)$. We let the components $a_{i}$ take values in the range $0,1, \ldots,\left(N_{i}-1\right)$, and let the components $x^{\mu}$ take values in the range $0,1, \ldots,\left(N_{\mu}-1\right)$.

We begin by describing the dictionary between the two types of braiding statistics data, $\left\{R_{x y}, F_{x y z}\right\}$ and $\left\{\Phi_{\mu}, \Phi_{\mu \nu}\right\}$. One direction is easy: it is clear from the definitions of $\Phi_{\mu}$ and $\Phi_{\mu \nu}$ that

$$
e^{-i \Phi_{\mu}}=R_{\boldsymbol{\epsilon}_{\mu} \boldsymbol{\epsilon}_{\mu}}, \quad e^{-i \Phi_{\mu \nu}}=R_{\boldsymbol{\epsilon}_{\mu} \boldsymbol{\epsilon}_{\nu}} R_{\boldsymbol{\epsilon}_{\nu} \boldsymbol{\epsilon}_{\mu}},
$$

where $\boldsymbol{\epsilon}_{\mu}$ denotes the unit type- $\mu$ anyon, $(0, \ldots, 1, \ldots, 0)$ with a 1 in the $\mu$ th entry. [Here, the " - " sign in the exponent is not particularly significant and depends on conventions: the sign can be either "+" or "-" depending on whether $R$ is defined in terms of counterclockwise or clockwise braiding. The reason we choose "-" rather than "+" is that, with this convention, the bulk boundary formulas Eqs. (30)-(32) are consistent with the cohomology formula Eq. (61). If we choose the "+" sign instead, then Eqs. (30)(32) are consistent with a modified version of Eq. (61) which is obtained by replacing $\nu \rightarrow \nu^{-1}$.]

The opposite direction, in which we express $\left\{R_{x y}, F_{x y z}\right\}$ in terms of $\left\{\Phi_{\mu}, \Phi_{\mu \nu}\right\}$, is harder. One problem is that there are some gauge choices in the definition of $\left\{R_{x y}, F_{x y z}\right\}$, so the inverse map is not uniquely defined. Another problem is that there are complicated constraints on $\left\{R_{x y}, F_{x y z}\right\}$ coming from the pentagon and hexagon equations [47]. Thus, we not only have to invert Eq. (64), but we also have to solve these constraints. Fortunately, both of these problems can be overcome by doing things in the right order. In particular, we proceed by first finding the most general solution $\left\{R_{x y}, F_{x y z}\right\}$ to the pentagon and hexagon 
equations [47] that is consistent with the fusion rules specified by $A=\prod_{\mu=1}^{M} \mathbb{Z}_{N_{\mu}}$, and then matching the general expression for $R_{x y}$ to Eq. (64). Skipping over the intermediate steps, the final result is that we obtain the following expressions for $\left\{R_{x y}, F_{x y z}\right\}$ :

$$
\begin{aligned}
R_{x y} & =\exp \left(-i \sum_{\mu} x^{\mu} y^{\mu} \Phi_{\mu}-i \sum_{\mu<\nu} x^{\mu} y^{\nu} \Phi_{\mu \nu}\right), \\
F_{x y z} & =\exp \left(-i \sum_{\mu} x^{\mu}\left(y^{\mu}+z^{\mu}-\left[y^{\mu}+z^{\mu}\right]\right) \Phi_{\mu}\right) .
\end{aligned}
$$

Here, the square bracket $\left[y^{\mu}+z^{\mu}\right]$ is defined to be $y^{\mu}+z^{\mu}$ $\left(\bmod N_{\mu}\right)$ with values taken in the range $0, \ldots, N_{\mu}-1$. The two mappings [Eqs. (64) and (65)] define a one-to-one correspondence between the data $\left\{\Phi_{\mu}, \Phi_{\mu \nu}\right\}$ and $\left\{R_{x y}, F_{x y z}\right\}$ (up to gauge equivalence).

Next, we turn to the connection between $\omega$ and $\left\{\Omega_{i \mu}, \Omega_{i j \mu}, x_{i l}^{\mu}\right\}$. In order to make this connection, it is helpful to introduce another quantity, namely, a unitary matrix $U_{a}(x)$ that is associated to each surface anyon $x \in A$ and each group element $a \in G$. This matrix $U_{a}(x)$ is defined as follows [36,37,47,51]. Recall that the surface anyons are all Abelian (by assumption), so they do not have any topologically protected degeneracies. However, they can have symmetry-protected degeneracies; that is, the surface anyons can have multiple internal states that are degenerate with one another and that cannot be split without breaking one of the symmetries. As a consequence of this degeneracy, if we braid an anyon $x$ around a defect line $a$, then the resulting Berry phase can be non-Abelian since the internal states of $x$ can mix with one another. We define the matrix $U_{a}(x)$ to be the (possibly non-Abelian) Berry phase associated with this braiding process.

An important property of the $U_{a}(x)$ matrices is that they obey the following relation $[37,51]$ :

$U_{a}(x) U_{b}(x)=\exp \left(i \sum_{\mu \nu} x^{\mu} \omega^{\nu}(a, b) \Phi_{\mu \nu}\right) U_{a+b}(x)$.

Here, $\omega^{\nu}(a, b)$ denotes the $\nu$ th component of $\omega(a, b)$, which we think of as an $M$-component integer vector. To derive this relation, imagine we braid an anyon $x$ around a type- $b$ defect line and then around a type- $a$ defect line. We can compute the Berry phase associated with this process in two different ways. In the first approach, we simply compose the two braiding processes, giving a Berry phase $U_{a}(x) \cdot U_{b}(x)$. In the second approach, we fuse the two defect lines together to form a type $a+b$ defect line together with an additional surface anyon $\omega(a, b)$. The Berry phase is therefore equal to $e^{i \sum_{\mu \nu} x^{\mu} \omega^{\nu}(a, b)} \Phi_{\mu \nu} U_{a+b}(x)$, where the first factor comes from the statistical phase associated with braiding $x$ around the anyon $\omega(a, b)$.
Demanding consistency between these two calculations gives Eq. (66).

With the help of Eq. (66), we can now derive the connection between $\omega$ and $\left\{\Omega_{i \mu}, \Omega_{i j \mu}, x_{i l}^{\mu}\right\}$. Let us start by expressing $\Omega_{i \mu}$ in terms of $\omega$. Recall that $\Omega_{i \mu}$ is defined as the Berry phase associated with braiding a type- $\epsilon_{\mu}$ anyon around an $\mathbf{e}_{i}$ gauge flux for $N^{i \mu}$ times. Equivalently, given the connection between gauge fluxes and defect lines, $\Omega_{i \mu}$ is equal to the Berry phase associated with braiding a type$\epsilon_{\mu}$ anyon around a type- $\mathbf{e}_{i}$ defect line for $N^{i \mu}$ times. Expressing the latter Berry phase in terms of the $U_{a}(x)$ matrices, we derive

$$
e^{i \Omega_{i \mu}}=\left(U_{\mathbf{e}_{i}}\left(\epsilon_{\mu}\right)\right)^{N^{i \mu}} .
$$

Next, we express the right-hand side of Eq. (67) in terms of $\omega$ by using Eq. (66) repeatedly:

$$
\left(U_{\mathbf{e}_{i}}\left(\epsilon_{\mu}\right)\right)^{N^{i \mu}}=\exp \left(i \sum_{\nu} \sum_{n=1}^{N^{i \mu}} \omega^{\nu}\left(\mathbf{e}_{i}, n \mathbf{e}_{i}\right) \Phi_{\mu \nu}\right) .
$$

Combining Eqs. (67) and (68), we conclude that

$$
\Omega_{i \mu}=\sum_{\nu} \sum_{n=1}^{N^{i \mu}} \omega^{\nu}\left(\mathbf{e}_{i}, n \mathbf{e}_{i}\right) \Phi_{\mu \nu} .
$$

In a similar fashion, we can express $\Omega_{i j \mu}$ in terms of $\omega$. First, we note that $\Omega_{i j \mu}$ is equal to the Berry phase associated with braiding a type- $\epsilon_{\mu}$ anyon around a type$\mathbf{e}_{i}$ defect and then around a type-e $\mathbf{e}_{j}$ defect and then around the type- $\mathbf{e}_{i}$ defect in the opposite direction and finally around the type- $\mathbf{e}_{j}$ defect in the opposite direction. Expressing this Berry phase in terms of the $U_{a}(x)$ matrices gives

$$
e^{i \Omega_{i j \mu}}=U_{\mathbf{e}_{j}}\left(\epsilon_{\mu}\right)^{-1} U_{\mathbf{e}_{i}}\left(\epsilon_{\mu}\right)^{-1} U_{\mathbf{e}_{j}}\left(\epsilon_{\mu}\right) U_{\mathbf{e}_{i}}\left(\epsilon_{\mu}\right) .
$$

As before, we can now rewrite the right-hand side in terms of $\omega$ to obtain

$$
\Omega_{i j \mu}=\sum_{\nu}\left[\omega^{\nu}\left(\mathbf{e}_{j}, \mathbf{e}_{i}\right)-\omega^{\nu}\left(\mathbf{e}_{i}, \mathbf{e}_{j}\right)\right] \Phi_{\mu \nu} .
$$

Finally, we need to express $x_{i l}^{\mu}$ in terms of $\omega$. Comparing the expression for $\Omega_{i j \mu}$ with the definition of $x_{i l}^{\mu}$, Eq. (18), we see that

$$
x_{i l}^{\mu}=\omega^{\mu}\left(\mathbf{e}_{l}, \mathbf{e}_{i}\right)-\omega^{\mu}\left(\mathbf{e}_{i}, \mathbf{e}_{l}\right) .
$$

One can check that the expressions Eqs. (69), (71), and (72) are all invariant under the gauge transformation Eq. (63).

It is also possible to translate in the opposite direction; that is, we can express $\omega$ in terms of $\left\{\Omega_{i \mu}, \Omega_{i j \mu}, x_{i l}^{\mu}\right\}$ for a particular gauge choice. However, we do not need this 
expression in what follows, so we do not write it down explicitly. Instead, we only need the fact that Eqs. (69), (71), and (72) define a one-to-one correspondence between $\left\{\Omega_{i \mu}, \Omega_{i j \mu}, x_{i l}^{\mu}\right\}$ and $\left\{\omega \in H^{2}(G, A)\right\}$. We establish this result in Appendix H.

\section{Translating between the two types of bulk data}

Next, we review how to compute the bulk data $\left\{\Theta_{i, l}, \Theta_{i j, l}, \Theta_{i j k, l}\right\}$ for a group cohomology model with 4-cocycle $\nu$. This calculation was worked out in Ref. [38]. The result is

$$
\begin{gathered}
e^{i \Theta_{i, l}}=\prod_{n=1}^{N_{i}} \chi_{\mathbf{e}_{l}, \mathbf{e}_{i}}\left(\mathbf{e}_{i}, n \mathbf{e}_{i}\right), \\
e^{i \Theta_{i j, l}}=\prod_{n=1}^{N^{i j}} \chi_{\mathbf{e}_{l}, \mathbf{e}_{i}}\left(\mathbf{e}_{j}, n \mathbf{e}_{j}\right) \chi_{\mathbf{e}_{l}, \mathbf{e}_{j}}\left(\mathbf{e}_{i}, n \mathbf{e}_{i}\right), \\
e^{i \Theta_{i j k, l}}=\frac{\chi_{\mathbf{e}_{l}, \mathbf{e}_{i}}\left(\mathbf{e}_{k}, \mathbf{e}_{j}\right)}{\chi_{\mathbf{e}_{l}, \mathbf{e}_{i}}\left(\mathbf{e}_{j}, \mathbf{e}_{k}\right)}
\end{gathered}
$$

where

$$
\chi_{\mathbf{e}_{l}, \mathbf{e}_{i}}(b, c)=\frac{\chi_{\mathbf{e}_{l}}\left(\mathbf{e}_{i}, b, c\right) \chi_{\mathbf{e}_{l}}\left(b, c, \mathbf{e}_{i}\right)}{\chi_{\mathbf{e}_{l}}\left(b, \mathbf{e}_{i}, c\right)}
$$

and

$$
\chi_{a}(b, c, d)=\frac{\nu(b, a, c, d) \nu(b, c, d, a)}{\nu(a, b, c, d) \nu(b, c, a, d)} .
$$

Importantly, Ref. [38] showed that the bulk data $\left\{\Theta_{i, l}, \Theta_{i j, l}, \Theta_{i j k, l}\right\}$ uniquely distinguish every group cohomology model; in other words, the mapping between $\left\{\Theta_{i, l}, \Theta_{i j, l}, \Theta_{i j k, l}\right\}$ and $\nu$ is a one-to-one correspondence (up to gauge equivalence).

\section{Establishing the equivalence}

With the help of the dictionaries described above, we are now ready to compare the cohomology formula Eq. (61) to the bulk-boundary formulas, Eqs. (30)-(32). Our strategy is to translate Eq. (61) into three equations relating $\left\{\Theta_{i, l}, \Theta_{i j, l}, \Theta_{i j k, l}\right\}$ and $\left\{\Phi_{\mu}, \Phi_{\mu \nu}, \Omega_{i \mu}, \Omega_{i j \mu}, x_{i l}^{\mu}\right\}$. We then compare these equations directly to Eqs. (30)-(32).

We begin with the equation for $\Theta_{i, l}$. To derive this equation, we first express $\Theta_{i, l}$ in terms of $\nu$ using Eqs. (73), (76), and (77):

$$
e^{i \Theta_{i, l}}=\prod_{n=1}^{N_{i}} \frac{\nu\left(\mathbf{e}_{i}, n \mathbf{e}_{i}, \mathbf{e}_{i}, \mathbf{e}_{l}\right) \nu\left(\mathbf{e}_{i}, \mathbf{e}_{l}, n \mathbf{e}_{i}, \mathbf{e}_{i}\right)}{\nu\left(\mathbf{e}_{i}, n \mathbf{e}_{i}, \mathbf{e}_{l}, \mathbf{e}_{i}\right) \nu\left(\mathbf{e}_{l}, \mathbf{e}_{i}, n \mathbf{e}_{i}, \mathbf{e}_{i}\right)} .
$$

Next, we substitute Eq. (61) into the right-hand side. Since the resulting expression is complicated, it is helpful to separately evaluate the contributions from the $R$ and $F$ symbols. We start with the contribution from the $R$ symbol:

$$
e^{i \Theta_{i, l}}[R]=\prod_{n=1}^{N_{i}} \frac{R_{\omega\left(\mathbf{e}_{i}, \mathbf{e}_{l}\right), \omega\left(\mathbf{e}_{i}, n \mathbf{e}_{i}\right)} R_{\omega\left(n \mathbf{e}_{i}, \mathbf{e}_{i}\right), \omega\left(\mathbf{e}_{i}, \mathbf{e}_{l}\right)}}{R_{\omega\left(\mathbf{e}_{l}, \mathbf{e}_{i}\right), \omega\left(\mathbf{e}_{i}, n \mathbf{e}_{i}\right)} R_{\omega\left(n \mathbf{e}_{i}, \mathbf{e}_{i}\right), \omega\left(\mathbf{e}_{l}, \mathbf{e}_{i}\right)}} .
$$

Ultimately, we want to express the quantities on the righthand side in terms of the surface data $\left\{\Phi_{\mu}, \Phi_{\mu \nu}, \Omega_{i \mu}\right.$, $\left.\Omega_{i j \mu}, x_{i l}^{\mu}\right\}$. However, rather than doing this immediately, it is convenient to first use the identity [55]

$$
\omega\left(n \mathbf{e}_{i}, \mathbf{e}_{i}\right)=\omega\left(\mathbf{e}_{i}, n \mathbf{e}_{i}\right)
$$

to rewrite the above expression as

$$
e^{i \Theta_{i, l}}[R]=\prod_{n=1}^{N_{i}} \frac{R_{\omega\left(\mathbf{e}_{i}, \mathbf{e}_{l}\right), \omega\left(\mathbf{e}_{i}, n \mathbf{e}_{i}\right)} R_{\omega\left(\mathbf{e}_{i}, n \mathbf{e}_{i}\right), \omega\left(\mathbf{e}_{i}, \mathbf{e}_{l}\right)}}{R_{\omega\left(\mathbf{e}_{l}, \mathbf{e}_{i}\right), \omega\left(\mathbf{e}_{i}, n \mathbf{e}_{i}\right)} R_{\omega\left(\mathbf{e}_{i}, n \mathbf{e}_{i}\right), \omega\left(\mathbf{e}_{l}, \mathbf{e}_{i}\right)}}
$$

We can then express the product of $R$ symbols in terms of $\Phi_{\mu \nu}$ using

$$
R_{x y} R_{y x}=\exp \left(-i \theta_{x y}\right)=\exp \left(-i \sum_{\mu \nu} x^{\mu} y^{\nu} \Phi_{\mu \nu}\right),
$$

thus giving

$$
e^{i \Theta_{i, l}[R]}=\exp \left\{i \sum_{n=1}^{N_{i}} \sum_{\mu \nu} X_{\mathbf{e}_{i}, \mathbf{e}_{l}}^{\mu} \omega^{\nu}\left(\mathbf{e}_{i}, n \mathbf{e}_{i}\right) \Phi_{\mu \nu}\right\}
$$

where $X_{a b}^{\mu} \equiv \omega^{\mu}(b, a)-\omega^{\mu}(a, b)$. Identifying $X_{\mathbf{e}_{i}, \mathbf{e}_{l}}^{\mu}$ with $x_{i l}^{\mu}$ [see Eq. (72)], and comparing with the expression for $\Omega_{i \mu}$ Eq. (69), we derive

$$
e^{i \Theta_{i, l}}[R]=\exp \left(i \sum_{\mu} \frac{N_{i}}{N^{i \mu}} x_{i l}^{\mu} \Omega_{i \mu}\right) .
$$

Now consider the contribution from the $F$ symbols,

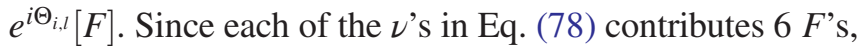
the expression for $e^{i \Theta_{i, l}}[F]$ contains $24 F$ 's. We need to relate this product of $24 F$ 's to the surface data $\left\{\Phi_{\mu}, \Phi_{\mu \nu}, \Omega_{i \mu}, \Omega_{i j \mu}, x_{i l}^{\mu}\right\}$. To do this, we express each $F$ in terms of $\Phi_{\mu}, \Phi_{\mu \nu}$ using Eq. (65), and then simplify the resulting expression. After some straightforward but tedious algebra, we obtain

$$
e^{i \Theta_{i, l}[F]}=\exp \left\{i \sum_{\mu} N_{i} \Phi_{\mu}\left(X_{\mathbf{e}_{i}, \mathbf{e}_{l}}^{\mu}\right)^{2}\right\} .
$$

Combining the $R$ and $F$ contributions and using the fact that $X_{\mathbf{e}_{i}, \mathbf{e}_{l}}^{\mu}=x_{i l}^{\mu}$, we finally derive an expression for $\Theta_{i, l}$ : 


$$
e^{i \Theta_{i, l}}=\exp \left\{i \sum_{\mu} \frac{N_{i}}{N^{i \mu}} x_{i l}^{\mu} \Omega_{i \mu}+N_{i}\left(x_{i l}^{\mu}\right)^{2} \Phi_{\mu}\right\} .
$$

At this point we can see that the above expression for $\Theta_{i, l}$ is identical to the bulk-boundary formula Eq. (30). In a similar fashion, we can show that the formulas for $\Theta_{i j, l}$ and $\Theta_{i j k, l}$ also match the corresponding bulk-boundary formulas Eqs. (31) and (32). Since the expressions for $\left\{\Theta_{i, l}, \Theta_{i j, l}, \Theta_{i j k, l}\right\}$ all match, we conclude that the cohomology formula Eq. (61) and the bulk-boundary formulas Eqs. (30)-(32) give equivalent constraints for the surfaces of the group cohomology models.

\section{CONCLUSION}

In summary, we derive a bulk-boundary correspondence for 3D bosonic SPT phases with finite unitary Abelian symmetry group. Our correspondence relates the bulk properties of these phases to the properties of their gapped symmetric surfaces. This relationship is described by three equations, Eqs. (30)-(32), which express the bulk data $\left\{\Theta_{i, l}, \Theta_{i j, l}, \Theta_{i j k, l}\right\}$ in terms of the surface data $\left\{\Phi_{\mu}, \Phi_{\mu \nu}\right.$, $\left.\Omega_{i \mu}, \Omega_{i j \mu}, x_{i l}^{\mu}\right\}$.

It should be possible to generalize our bulk-boundary correspondence beyond the case considered in this paper. For example, we make the simplifying assumption that the surface supports only Abelian anyons. However, there is reason to think that this assumption is actually unnecessary - that is, the bulk-boundary formulas Eqs. (30)-(32) continue to hold even if the surface supports nonAbelian anyons [56]. One piece of evidence for this is the close connection between Eqs. (30)-(32) and the conjecture Eq. (61) of Ref. [20]. Indeed, the conjecture Eq. (61) is believed to hold whether or not the surface supports non-Abelian anyons, suggesting that our correspondence should hold more generally as well.

Another natural extension of this work would be to fermionic SPT phases. It is not hard to see that two of the bulk-boundary formulas, Eqs. (31) and (32), generalize trivially to the fermionic case since they do not involve exchange statistics in any way. On the other hand, the formula Eq. (30) likely needs to be modified since this relation makes use of exchange statistics and topological spin.

An interesting direction for future work would be to study bulk-boundary relations for 3D SPT phases with gapless surfaces. A recent paper [57] proposed a bulkboundary correspondence in this context by considering modular transformations on a three-dimensional torus; it would be interesting to understand the relationship between this correspondence and the bulk-boundary relations derived in this paper.

\section{ACKNOWLEDGMENTS}

We thank M. Cheng and Z.-C. Gu for helpful discussions. This work is supported in part by the NSF under
Grant No. DMR-1254741. Research at Perimeter Institute is supported by the Government of Canada through Industry Canada and by the Province of Ontario through the Ministry of Economic Development \& Innovation. C.-H. L. acknowledges the funding from the Canada Research Chair (CRC) program and the University of Alberta.

\section{APPENDIX A: SHOWING $\Omega_{i \mu}$ AND $\Omega_{i j \mu}$ ARE WELL DEFINED}

\section{1. $\Omega_{i \mu}$ is well defined}

Recall that $\Omega_{i \mu}$ is defined as the Berry phase associated with braiding a surface anyon $\mathcal{X}$ around a vortex line $\alpha$ for $N^{i \mu}$ times. Here, $\mathcal{X}$ is any surface anyon carrying unit type$\mu$ anyonic flux, while $\alpha$ is any vortex line carrying unit type$i$ gauge flux. The goal of this section is to show that this definition is sensible, that is, (1) the above Berry phase is Abelian and (2) the Berry phase does not depend on the choice of $\mathcal{X}$ or $\alpha$.

To establish these points, we analyze the above braiding process using a diagrammatic technique. This diagrammatic technique was originally developed for $2 \mathrm{D}$ anyon systems; in order to apply it to our system, we imagine folding the surface and straightening the vortex line $\alpha$ as in Fig. 7. After doing this, we can view our system as two dimensional, and we can view $\alpha$ and $\mathcal{X}$ as 2D anyons. We will assume this quasi-2D point of view in what follows.

While the diagrammatic method has sophisticated rules, we only need a few ingredients in our proof. (For more details about the diagrammatic method, see Ref. [47].) The diagrams that we use are built out of lines and trivalent vertices and are drawn in a $(2+1)$-dimensional space. Roughly speaking, the lines represent space-time trajectories of $2 \mathrm{D}$ anyons with the arrow of time being upward, while the vertices are where the anyons fuse or split. Each diagram defines a complex number or matrix, which can be interpreted as the quantum mechanical amplitude for the process shown in the diagram. These amplitudes can be evaluated using certain relations. What we need below are the following two relations:



where $\bar{a}$ is the antiparticle of an anyon $a$ and $u_{a}$ is a complex number associated with $a$, and 


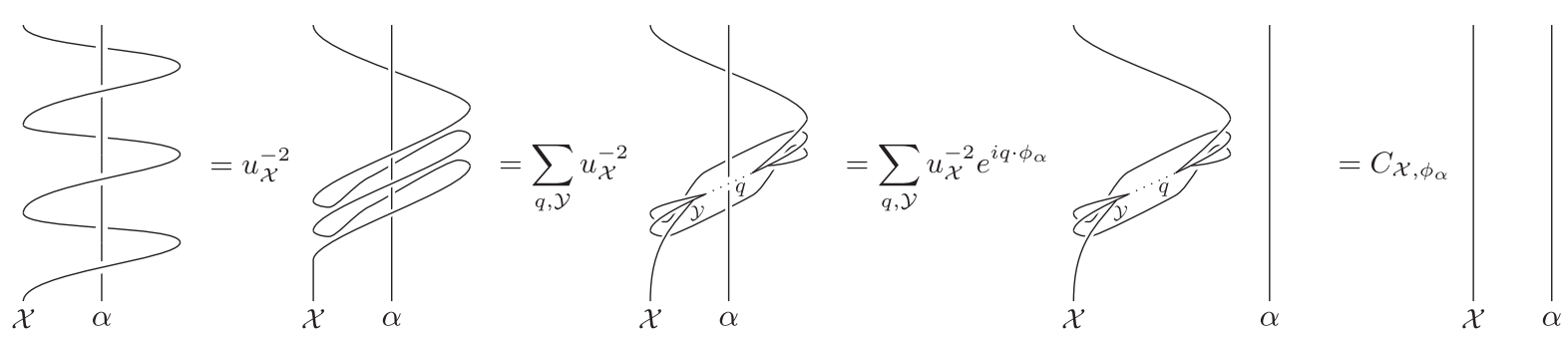

FIG. 12. Diagrammatic proof that $\Omega_{i \mu}$ is well defined.

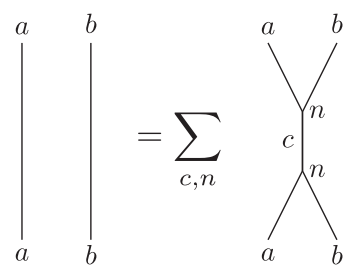

where $n$ labels the states in the fusion space $\mathbb{V}_{a b}^{c}$, i.e., the different ways to split $c$ into $a$ and $b$.

With this background, we are now ready to establish points (1) and (2) listed above. The main calculation is shown in Fig. 12. We start with the diagram on the far left, which shows a braiding process in which $\mathcal{X}$ is braided around $\alpha$ for $N^{i \mu}$ times. (The case $N^{i \mu}=3$ is shown for illustration.) The first equation follows from Eq. (A1), while the second equation follows from Eq. (A2). Here, the index $\mathcal{Y}$ runs over the different fusion products of $\mathcal{X} \times \mathcal{X}$, while the index $q$ runs over the fusion products of $\mathcal{Y} \times \mathcal{X}$. Importantly, we know that $q$ must be a charge excitation, since if we fuse $\mathcal{X}$ with itself $N^{i \mu}$ times, the resulting fusion products are all charge excitations according to Eqs. (16) and (17). To derive the third equation, we note that the mutual statistics between the charge $q$ and the vortex $\alpha$ is Abelian and is given by the Aharonov-Bohm law, i.e., $\exp \left(i q \cdot \phi_{\alpha}\right)$; therefore, we can pass the charge $q$ worldline through the $\alpha$ worldline at the cost of introducing a factor of $\exp \left(i q \cdot \phi_{\alpha}\right)$. Moving on to the last equation, we observe that in the fourth diagram of Fig. 12, the $\alpha$ worldline is completely decoupled from $\mathcal{X}$, but $\mathcal{X}$ still has some "self-interaction." This self-interaction may contribute a complicated numerical factor to the amplitude, but no matter how complicated it is, we can see that it depends only on $\mathcal{X}, \mathcal{Y}$, and $q$, and not on the fusion channel of $\mathcal{X}$ and $\alpha$. Therefore, after we perform the sum over $\mathcal{Y}$ and $q$, we can group all the numerical factors into a single complex number $C_{\mathcal{X}, \phi_{\alpha}}$ and thereby derive the last equation in Fig. 12. We note that since the braiding process is unitary, the constant $C_{\mathcal{X}, \phi_{\alpha}}$ must be a phase factor.

How does this calculation help us establish the two claims (1) and (2)? The key point is that $C_{\mathcal{X}, \phi_{\alpha}}$ depends on only $\mathcal{X}$ and $\alpha$ and does not depend on their fusion channel. This means that the braid matrix associated with this process is proportional to the identity matrix; i.e., it is an Abelian phase. This establishes property (1). To establish (2) we need to show that the phase factor $C_{\mathcal{X}, \phi_{\alpha}}$ is independent of the choice of $\mathcal{X}$ or $\alpha$ as long as $\mathcal{X}$ carries unit type- $\mu$ anyonic flux and $\alpha$ carries unit type- $i$ gauge flux. It is obvious that $C_{\mathcal{X}, \phi_{\alpha}}$ is independent of the choice of $\alpha$ : to see that it is independent of the choice of $\mathcal{X}$, let $\mathcal{X}^{\prime}$ be another anyon that carries unit type- $\mu$ anyonic flux. We need to show that $C_{\mathcal{X}^{\prime}, \phi_{\alpha}}=C_{\mathcal{X}, \phi_{\alpha}}$. To prove this, we use the fact that $\mathcal{X}^{\prime}$ can be obtained by fusing some charge $q$ to $\mathcal{X}$. Then, by the Aharonov-Bohm law, we have

$$
C_{\mathcal{X}^{\prime}, \phi_{\alpha}}=C_{\mathcal{X}, \phi_{\alpha}} e^{i q \cdot \phi_{\alpha} N^{i \mu}}=C_{\mathcal{X}, \phi_{\alpha}},
$$

where the last equality follows from the fact that $N^{i \mu} \phi_{\alpha}$ is a multiple of $2 \pi$.

\section{2. $\Omega_{i j \mu}$ is well defined}

Recall that $\Omega_{i j \mu}$ is defined as the Berry phase associated with the following process: a surface anyon $\mathcal{X}$ is first braided around a vortex $\alpha$, then around a vortex $\beta$, then around $\alpha$ in the opposite direction, and finally around $\beta$ in the opposite direction. Here, $\mathcal{X}$ is any surface anyon carrying unit type- $\mu$ anyonic flux, and $\alpha, \beta$ are any vortex lines carrying unit type- $i$ and type- $j$ gauge flux. Our goal is to show that this definition is sensible, that is, (1) the above Berry phase is Abelian and (2) the Berry phase does not depend on the choice of $\mathcal{X}, \alpha$, or $\beta$. As in the previous section, we establish these claims using the 2D diagrammatic technique; just as before, this $2 \mathrm{D}$ technique is applicable even though our system is three dimensional since we can fold the surface and straighten the vortex lines as in Fig. 7 so that $\mathcal{X}, \alpha$, and $\beta$ can be viewed as $2 \mathrm{D}$ anyons.

The main calculation is shown in Fig. 13. The diagram on the far left shows the space-time trajectories of $\mathcal{X}, \alpha, \beta$ during the above braiding process. The first equation follows from the relation Eq. (A1), while the second equation follows from the relation Eq. (A2), which we apply to the two shaded regions shown in the second diagram. Here, the indices $q, \tilde{q}$ run over the fusion products of $\mathcal{X} \times \overline{\mathcal{X}}$. Importantly $q$ and $\tilde{q}$ can only be charge excitations since all the fusion products of $\mathcal{X} \times \overline{\mathcal{X}}$ are charges according to Eqs. (16) and (17). To derive the third equation, we note that the Aharonov-Bohm law tells us that the mutual statistics between $q$ and $\beta$ is $\exp \left(i q \cdot \phi_{\beta}\right)$, while the statistics between $\tilde{q}$ and $\alpha$ is $\exp \left(i \tilde{q} \cdot \phi_{\alpha}\right)$; therefore, we 


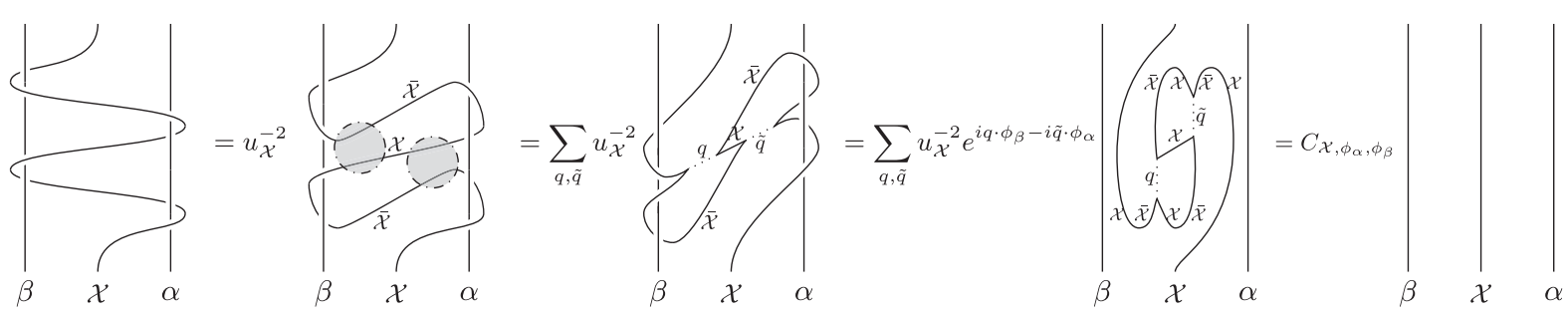

FIG. 13. Diagrammatic proof that $\Omega_{i j \mu}$ is well defined.

can pass the $q, \tilde{q}$ worldlines through the $\beta, \alpha$ worldlines at the cost of introducing factors of $\exp \left(i q \cdot \phi_{\beta}\right)$ and $\exp \left(-i \tilde{q} \cdot \phi_{\alpha}\right)$, respectively. Moving on to the last equation, we note that in the fourth diagram of Fig. 13, the $\alpha, \beta$ worldlines are completely decoupled from $\mathcal{X}$, but $\mathcal{X}$ still has some selfinteraction. By the same arguments as in the previous section, the contribution from this self-interaction can be grouped together with the other numerical factors into an overall constant $C_{\mathcal{X}, \phi_{\alpha}, \phi_{\beta}}$. In this way, we derive the last equation in Fig. 13. We note that since the braiding process is unitary, the constant $C_{\mathcal{X}, \phi_{\alpha}, \phi_{\beta}}$ must be a phase factor.

We are now ready to prove the two claims (1) and (2). To prove (1), we observe that $C_{\mathcal{X}, \phi_{\alpha}, \phi_{\beta}}$ does not depend on the fusion channel between $\mathcal{X}, \alpha, \beta$. This implies that the braid matrix associated with this process is proportional to the identity matrix; i.e., it is an Abelian phase. To prove (2), we need to show that the phase factor $C_{\mathcal{X}, \phi_{\alpha}, \phi_{\beta}}$ is independent of the choice of $\mathcal{X}, \alpha$, or $\beta$ as long as $\mathcal{X}$ carries unit type- $\mu$ anyonic flux and $\alpha, \beta$ carry unit type- $i$ and type- $j$ gauge flux. It is obvious that $C_{\mathcal{X}, \phi_{\alpha}, \phi_{\beta}}$ is independent of the choice of $\alpha, \beta$ : to see that it is independent of the choice of $\mathcal{X}$, let $\mathcal{X}^{\prime}$ be another anyon that carries unit type- $\mu$ anyonic flux. We need to show that $C_{\mathcal{X}^{\prime}, \phi_{\alpha}, \phi_{\beta}}=C_{\mathcal{X}, \phi_{\alpha}, \phi_{\beta}}$. To prove this, we note that $\mathcal{X}^{\prime}$ can be obtained by fusing some charge $q$ to $\mathcal{X}$. Therefore, by the Aharonov-Bohm law, we have

$$
\begin{aligned}
C_{\mathcal{X}^{\prime}, \phi_{\alpha}, \phi_{\beta}} & =C_{\mathcal{X}, \phi_{\alpha}, \phi_{\beta}} e^{i q \cdot \phi_{\alpha}+i q \cdot \phi_{\beta}-i q \cdot \phi_{\alpha}-i q \cdot \phi_{\beta}} \\
& =C_{\mathcal{X}, \phi_{\alpha}, \phi_{\beta}} .
\end{aligned}
$$

This establishes claim (2).

\section{APPENDIX B: PHYSICAL INTERPRETATION OF $\boldsymbol{x}_{i l}$}

In Sec. III D, we give a physical interpretation of the quantity $x_{i l}$ in terms of the thought experiment depicted in Fig. 4. More precisely, we make the claim that [see Eq. (20) in the main text]

$$
\begin{aligned}
& \xi_{\mathcal{X}}=\xi_{\mathcal{X}^{\prime}}=\cdots=x_{i l}, \\
& \xi_{\mathcal{S}}=\xi_{\mathcal{S}^{\prime}}=\cdots=-x_{i l},
\end{aligned}
$$

where $\mathcal{X}, \mathcal{X}^{\prime}, \ldots$ are the different surface anyons obtained by shrinking the arch $\alpha$ in Fig. 4(c), while $\mathcal{S}, \mathcal{S}^{\prime}, \ldots$ are the different surface anyons obtained by shrinking the arch $\sigma$. In this appendix, we prove Eq. (B1).

The key step in our derivation is to consider a process in which we braid a surface anyon $\mathcal{F}$ around the two vortex arches shown in Fig. 4(b):

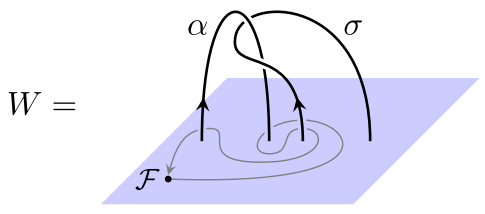

Assuming that $\mathcal{F}$ carries unit type- $\mu$ anyonic flux, what we show is that the above process gives rise to an Abelian Berry phase that is equal to $\Omega_{i l \mu}$. Equivalently, in more formal language, we show that

$$
W|\psi\rangle=e^{i \Omega_{i l \mu}}|\psi\rangle,
$$

where $|\psi\rangle$ denotes the initial state at the beginning of the braiding process.

Before we establish Eq. (B3), we now argue that it implies Eq. (B1). To see this, notice that the above braiding process $W$ can be smoothly deformed into a process in which $\mathcal{F}$ is braided around both ends of $\alpha$ in Fig. 4(c). The latter process can then be deformed into a process in which $\mathcal{F}$ is braided around the surface excitation obtained by shrinking down $\alpha$ [see Fig. 4(d)]. Since the Berry phase must remain unchanged during these deformations, it follows that

$$
e^{i \theta_{\mathcal{F X}}}|\psi\rangle=e^{i \theta_{\mathcal{F X}}}|\psi\rangle=\cdots=W|\psi\rangle,
$$

where $\mathcal{X}, \mathcal{X}^{\prime}, \ldots$ are the different surface anyons obtained by shrinking the $\operatorname{arch} \alpha$. At the same time, we can straightforwardly compute the statistical phase $\theta_{\mathcal{F} \mathcal{X}}$ using Eqs. (14) and (13):

$$
\theta_{\mathcal{F} \mathcal{X}}=\theta_{\xi_{\mathcal{F}} \xi_{\mathcal{X}}}=\sum_{\nu}\left(\xi_{\mathcal{X}}\right)^{\nu} \Phi_{\mu \nu}
$$

Similarly, we have

$$
\theta_{\mathcal{F} \mathcal{X}^{\prime}}=\sum_{\nu}\left(\xi_{\mathcal{X}^{\prime}}\right)^{\nu} \Phi_{\mu \nu}
$$


and so on. Putting together Eqs. (B3)-(B6), we derive

$$
\sum_{\nu}\left(\xi_{\mathcal{X}}\right)^{\nu} \Phi_{\mu \nu}=\sum_{\nu}\left(\xi_{\mathcal{X}^{\prime}}\right)^{\nu} \Phi_{\mu \nu}=\cdots=\Omega_{i l \mu} \quad(\bmod 2 \pi) .
$$

We conclude that

$$
\left(\xi_{\mathcal{X}}\right)^{\nu}=\left(\xi_{\mathcal{X}^{\prime}}\right)^{\nu}=\cdots=x_{i l}^{\nu},
$$

where $x_{i l}^{\nu}$ is the unique integer solution to

$$
\sum_{\nu} x_{i l}^{\nu} \Phi_{\mu \nu}=\Omega_{i l \mu} \quad(\bmod 2 \pi) .
$$

This establishes the first relation in Eq. (B1).

As for the second relation in Eq. (B1), this follows from the fact that if we further fuse the two surface anyons in Fig. 4(d), the outcome must be a charge excitation or the vacuum; hence, $\mathcal{S}, \mathcal{S}^{\prime}, \ldots$ etc. must carry opposite anyonic flux to $\mathcal{X}, \mathcal{X}^{\prime}, \ldots$ etc.

All that remains is to derive Eq. (B3). To do this, we decompose $W$ into simpler processes. In particular, let
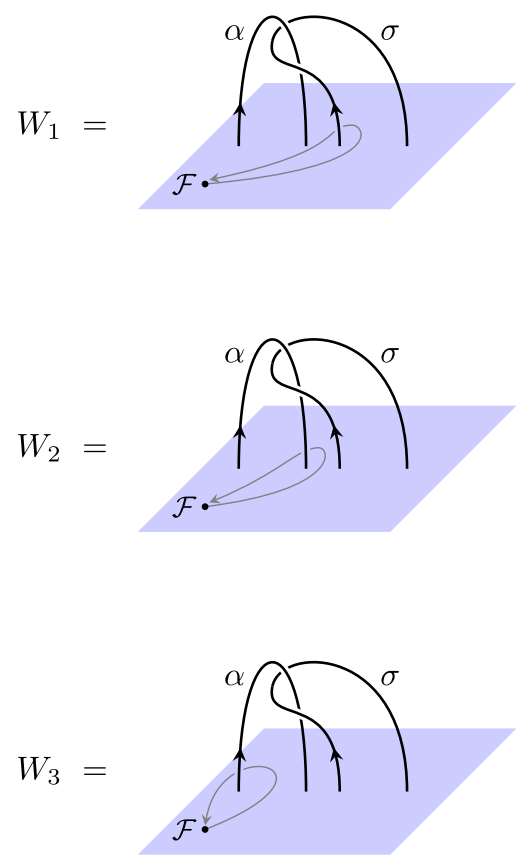

With these definitions, we can see that

$$
W=W_{3} W_{1}^{-1} W_{2} W_{1} .
$$

Next, we observe that $W_{1}$ and $W_{2}$ obey the commutation relation

$$
W_{2}^{-1} W_{1}^{-1} W_{2} W_{1}=e^{-i \Omega_{l i \mu} \hat{I}} .
$$

This relation follows from the definition of $\Omega_{l i \mu}$, where the minus sign comes from the fact that $\mathcal{F}$ is braided around the "incoming-flux" end of $\alpha$, instead of the "outgoing-flux" end (see Fig. 3).
In addition, we have the relation

$$
W_{3} W_{2}|\psi\rangle=|\psi\rangle .
$$

To see this, note that $W_{3} W_{2}$ describes a process in which $\mathcal{F}$ is braided around both ends of $\alpha$ in the configuration shown in Fig. 4(b). This braiding must give a trivial Berry phase since we can deform the vortex arches from Fig. 4(b) back to Fig. 4(a) by fusing together the ends of $\alpha$ and lifting $\alpha$ off the surface, and this lifting process commutes with the braiding of $\mathcal{F}$.

Combining Eqs. (B10)-(B12), we derive

$$
W|\psi\rangle=\left(W_{3} W_{2}\right)\left(W_{2}^{-1} W_{1}^{-1} W_{2} W_{1}\right)|\psi\rangle=e^{-i \Omega_{l i \mu}}|\psi\rangle .
$$

Equation (B3) now follows immediately from the fact that $\Omega_{l i \mu}=-\Omega_{i l \mu}$.

\section{APPENDIX C: DERIVING EQ. (32)}

In this appendix, we derive the bulk-boundary formula Eq. (32). The derivation closely follows that of Eq. (31).

To begin, let us recall the braiding process that defines $\Theta_{i j k, l}$ : first, we braid a loop $\alpha$ around a $\operatorname{loop} \beta$, then we braid $\alpha$ around another loop $\gamma$, and then finally we braid $\alpha$ around $\beta$ and $\gamma$ in the opposite direction. Here, $\alpha, \beta, \gamma$ are linked with a base loop $\sigma$ and the four loops carry unit flux of type $i, j, \quad k, \quad l$, respectively; i.e., $\phi_{\alpha}=\left(2 \pi / N_{i}\right) \mathbf{e}_{i}, \quad \phi_{\beta}=$ $\left(2 \pi / N_{j}\right) \mathbf{e}_{j}, \phi_{\gamma}=\left(2 \pi / N_{k}\right) \mathbf{e}_{k}$, and $\phi_{\sigma}=\left(2 \pi / N_{l}\right) \mathbf{e}_{l}$.

The first step of the derivation is to deform the above process onto the surface using the same procedure as in Fig. 5. After this deformation, $\alpha, \beta, \gamma, \sigma$ become vortex arches, and the braiding process involves braiding the arch $\alpha$ around the $\operatorname{arch} \beta$, and then braiding $\alpha$ around $\gamma$, and then finally braiding $\alpha$ around $\beta$ and $\gamma$ in the opposite direction. By the same arguments as in Sec. VA, the Berry phase for this new process must be the same as for the old one; that is, it must be equal to $\Theta_{i j k, l}$.

In the next step, we split $\alpha$ into two excitations: a surface anyon $\mathcal{X}$ and a vortex arch $\tilde{\alpha}$. Similarly, we split $\beta$ into an anyon $\mathcal{Y}$ and an arch $\tilde{\beta}$, and we split $\gamma$ into an anyon $\mathcal{Z}$ and an $\operatorname{arch} \tilde{\gamma}$. Similarly to Sec. V B, we choose $\mathcal{X}, \mathcal{Y}$, and $\mathcal{Z}$ to be any anyons carrying anyonic flux

$$
\xi_{\mathcal{X}}=x_{i l}, \quad \xi_{\mathcal{Y}}=x_{j l}, \quad \xi_{\mathcal{Z}}=x_{k l},
$$

while we choose $\tilde{\alpha}, \tilde{\beta}$, and $\tilde{\gamma}$ to be any arches with the property that $\alpha$ can be written as a fusion product of $\mathcal{X}$ and $\tilde{\alpha}$, and similarly for $\tilde{\beta}, \tilde{\gamma}$. This splitting is designed so that $\tilde{\alpha}$, $\tilde{\beta}, \tilde{\gamma}$ have a special property; namely, if we shrink these arches to the surface, we get a superposition of charge excitations rather than more complicated surface anyons.

At this point our braiding process involves braiding the pair $\{\tilde{\alpha}, \mathcal{X}\}$ around $\{\tilde{\beta}, \mathcal{Y}\}$, and then around $\{\tilde{\gamma}, \mathcal{Z}\}$, and then finally around $\{\tilde{\beta}, \mathcal{Y}\}$ and $\{\tilde{\gamma}, \mathcal{Z}\}$ in the opposite 
direction. Following the same notation as in Sec. V C, let $|\psi\rangle$ denote the initial state at the beginning of the braiding, and let $U$ denote the braid matrix associated with this braiding. Then, in this notation, the fact that the Berry phase for this process is $\Theta_{i j k, l}$ translates to the equation

$$
U|\psi\rangle=e^{i \Theta_{i j k, l}}|\psi\rangle .
$$

Next, we write $U$ as

$$
U=U_{2}^{-1} U_{1}^{-1} U_{2} U_{1},
$$

where $U_{1}, U_{2}$ are the braid matrices corresponding to the following processes:
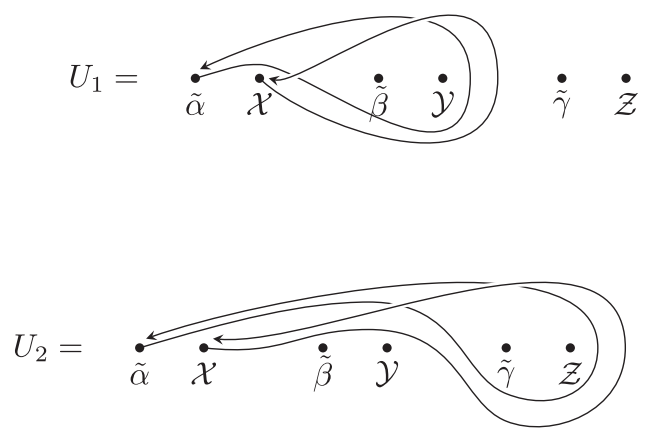

We then further decompose $U_{1}, U_{2}$ into simpler processes. In particular, define

$$
\begin{aligned}
& U_{\tilde{\alpha} \tilde{\beta}}=\underset{\tilde{\alpha}}{\stackrel{\dot{\mathcal{X}}}{\tilde{\beta}}} \dot{\mathcal{Y}} \quad \dot{\tilde{\gamma}} \quad \dot{\mathcal{Z}}
\end{aligned}
$$

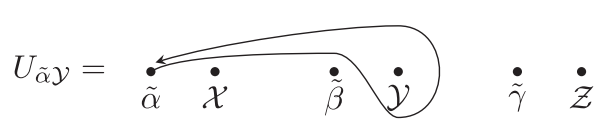

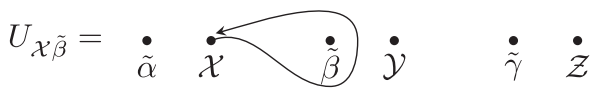

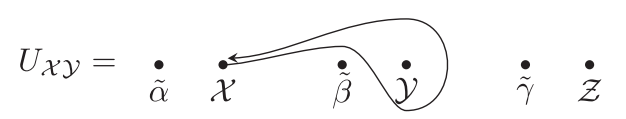

$$
\begin{aligned}
& U_{\tilde{\alpha} \tilde{\gamma}}=\begin{array}{llll}
\tilde{\tilde{\alpha}} & \dot{\mathcal{X}} & \dot{\tilde{\beta}} & \dot{\mathcal{Y}}
\end{array} \dot{\tilde{\gamma}}_{\dot{\mathcal{Z}}}
\end{aligned}
$$

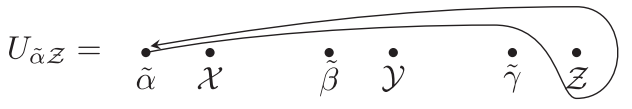

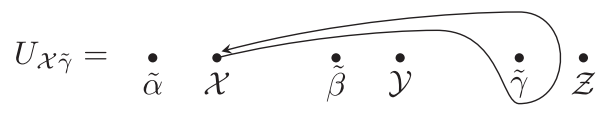

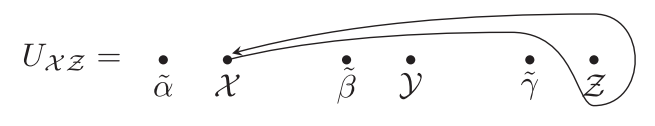

With these definitions, it is easy to see that

$$
\begin{aligned}
& U_{1}=U_{\mathcal{X Y}} U_{\mathcal{X} \tilde{\beta}} U_{\tilde{\alpha} \mathcal{Y}} U_{\tilde{\alpha} \tilde{\beta}}, \\
& U_{2}=U_{\mathcal{X Z}} U_{\mathcal{X} \tilde{\gamma}} U_{\tilde{\alpha} \mathcal{Z}} U_{\tilde{\alpha} \tilde{\gamma}} .
\end{aligned}
$$

Combining Eqs. (C2) and (C3), we conclude that

$$
\begin{aligned}
e^{i \Theta_{i j k, l}}|\psi\rangle= & \left(U_{\tilde{\alpha} \tilde{\gamma}}^{-1} U_{\tilde{\alpha} \mathcal{Z}}^{-1} U_{\mathcal{X} \tilde{\gamma}}^{-1} U_{\mathcal{X} \mathcal{Z}}^{-1}\right)\left(U_{\tilde{\alpha} \tilde{\beta}}^{-1} U_{\tilde{\alpha} \mathcal{Y}}^{-1} U_{\mathcal{X} \tilde{\beta}}^{-1} U_{\mathcal{X} \mathcal{Y}}^{-1}\right) \\
& \times\left(U_{\mathcal{X} \mathcal{Z}} U_{\mathcal{X} \tilde{\gamma}} U_{\tilde{\alpha} \mathcal{Z}} U_{\tilde{\alpha} \tilde{\gamma}}\right)\left(U_{\mathcal{X Y}} U_{\mathcal{X} \tilde{\beta}} U_{\tilde{\alpha} \mathcal{Y}} U_{\tilde{\alpha} \tilde{\beta}}\right)|\psi\rangle .
\end{aligned}
$$

The last step is to evaluate the expression on the righthand side of Eq. (C16). Our strategy is straightforward: since every operator $U$ appears along with its inverse $U^{-1}$, we use the commutation relations satisfied by these braid matrices to bring each $U$ and $U^{-1}$ next to one another and then cancel each pair. These commutation relations are as follows:

$$
\begin{array}{ll}
U_{\mathcal{X} \tilde{\gamma}} U_{\mathcal{X} \tilde{\beta}}=e^{i \eta_{1}} U_{\mathcal{X} \tilde{\beta}} U_{\mathcal{X} \tilde{\gamma}}, & U_{\tilde{\alpha} \tilde{\gamma}} U_{\tilde{\alpha} \mathcal{Y}}=e^{i \eta_{2}} U_{\tilde{\alpha} \mathcal{Y}} U_{\tilde{\alpha} \tilde{\gamma}}, \\
U_{\tilde{\alpha} \mathcal{Z}} U_{\tilde{\alpha} \tilde{\beta}}=e^{i \eta_{3}} U_{\tilde{\alpha} \tilde{\beta}} U_{\tilde{\alpha} \mathcal{Z}}, & U_{\mathcal{X} \tilde{\beta}} U_{\tilde{\alpha} \tilde{\beta}}=e^{i \eta_{4}} U_{\tilde{\alpha} \tilde{\beta}} U_{\mathcal{X} \tilde{\beta}}, \\
U_{\tilde{\alpha} \tilde{\beta}} U_{\tilde{\alpha} \mathcal{Y}}=e^{i \eta_{5}} U_{\tilde{\alpha} \mathcal{Y}} U_{\tilde{\alpha} \tilde{\beta}}, & U_{\tilde{\alpha} \tilde{\gamma}} U_{\tilde{\alpha} \mathcal{Z}}=e^{i \eta_{6}} U_{\tilde{\alpha} \mathcal{Z}} U_{\tilde{\alpha} \tilde{\gamma}},
\end{array}
$$

where

$$
\begin{array}{rlrl}
\eta_{1} & =\sum_{\mu} x_{i l}^{\mu} \Omega_{j k \mu}, & \eta_{2}=\sum_{\mu} x_{j l}^{\mu} \Omega_{k i \mu}, \\
\eta_{3}=\sum_{\mu} x_{k l}^{\mu} \Omega_{i j \mu}, & \eta_{4}=\sum_{\mu} x_{i l}^{\mu} \Omega_{j i \mu}, \\
\eta_{5}=\sum_{\mu} x_{j l}^{\mu} \Omega_{j i \mu}, & \eta_{6}=\sum_{\mu} x_{k l}^{\mu} \Omega_{k i \mu} .
\end{array}
$$

All other pairs of the $U$ 's commute with one another, with the only exception being $\left(U_{\tilde{\alpha} \tilde{\gamma}}, U_{\tilde{\alpha} \tilde{\beta}}\right)$ : this pair satisfies the weaker commutation relation 


$$
U_{\tilde{\alpha} \tilde{\gamma}} U_{\tilde{\alpha} \tilde{\beta}}|\psi\rangle=U_{\tilde{\alpha} \tilde{\beta}} U_{\tilde{\alpha} \tilde{\gamma}}|\psi\rangle .
$$

The derivations of Eq. (C17) are similar to those of Eqs. (46) and (47), which are given in Appendix E. Likewise, the derivation of Eq. (C19) is similar to that of Eq. (51).

Let us now use these commutation relations to derive the bulk-boundary formula. Inserting these relations into Eq. (C16), and commuting through the different $U$ matrices, we obtain

$$
\Theta_{i j k, l}=\eta_{1}+\eta_{2}+\eta_{3} .
$$

If we now examine the definitions of $\eta_{1}, \eta_{2}, \eta_{3}$, we see that this is precisely the bulk-boundary formula Eq. (32).

Before concluding, it is worth pointing out that the relations in Eq. (C17) are not as complicated as they appear. In particular, these relations all share a common structure: they all involve pairs $\left(U_{X Y}, U_{X^{\prime} Y^{\prime}}\right)$ in which (1) three of the four indices $\left\{X, Y, X^{\prime}, Y^{\prime}\right\}$ are distinct, (2) two of the three distinct indices are vortices $\tilde{\alpha}, \tilde{\beta}, \tilde{\gamma}$, and (3) the remaining distinct index is a surface anyon $\mathcal{X}, \mathcal{Y}, \mathcal{Z}$.

\section{APPENDIX D: DERIVING EQ. (30)}

In this appendix, we derive the bulk-boundary formula, Eq. (30), for $\Theta_{i, l}$. For the purposes of our derivation, it is helpful to separate the cases where $N_{i}$ is odd and $N_{i}$ is even.

The case where $N_{i}$ is odd is very simple: in this case, we can express $\Theta_{i, l}$ in terms of $\Theta_{i i, l}$ using the relation Eq. (10). Making use of the bulk-boundary formula for $\Theta_{i i, l}$ Eq. (31) together with the constraints Eqs. (22) and (27), the required formula Eq. (30) follows immediately.

Now consider the case where $N_{i}$ is even. To derive Eq. (30) in this case, we recall the alternative definition of $\Theta_{i, l}$ discussed at the end of Sec. II B: $\Theta_{i, l}$ is equal to $(-1)$ times the Berry phase associated with braiding a vortex loop $\alpha$ around its antivortex $\bar{\alpha}$ for $\left(N_{i} / 2\right)$ times while both $\alpha$ and $\bar{\alpha}$ are linked to another vortex loop $\sigma$. Here, $\alpha$ and $\sigma$ carry unit type- $i$ and type- $l$ flux, respectively; i.e., $\phi_{\alpha}=\left(2 \pi / N_{i}\right) \mathbf{e}_{i}, \phi_{\sigma}=\left(2 \pi / N_{l}\right) \mathbf{e}_{l}$. (Note that this alternative definition is applicable only in the case where $N_{i}$ is even.)

The first step of the derivation is to deform the above braiding process onto the surface following the same procedure as in Fig. 5. After the deformation, the vortex loops $\alpha, \bar{\alpha}$ become vortex arches, and the deformed braiding process involves braiding the arch $\alpha$ around its "antiarch" $\bar{\alpha}$ for $\left(N_{i} / 2\right)$ times [58]. By the same arguments as in Sec. V $\mathrm{A}$, the Berry phase for this process must be the same as the original process; i.e., it must be equal to $-\Theta_{i, l}$.

Let us translate this statement into more formal language. Following the notation of Sec. V C, let $|\psi\rangle$ be the initial state at the beginning of the braiding, and let $V$ be the braid matrix associated with braiding $\alpha$ around $\bar{\alpha}$ once. Then, the fact that the Berry phase for our process is $-\Theta_{i, l}$ translates into the equation

$$
V^{N_{i} / 2}|\psi\rangle=e^{-i \Theta_{i, l}}|\psi\rangle .
$$

To proceed further, we imagine folding the surface and straightening the vortex lines as shown in Fig. 7. After doing this, we then view the folded surface as a $2 \mathrm{D}$ system-a 2D slab. This point of view is convenient because it allows us to think about the braiding process as one involving $2 \mathrm{D}$ anyons, so we can make use of the powerful tools that have been developed for 2D systems. In particular, using these tools, one can show that (see Appendix F)

$$
V|\psi\rangle=e^{-4 \pi i s_{\alpha}}|\psi\rangle,
$$

where $s_{\alpha}$ is the topological spin of $\alpha$ when viewed as a $2 \mathrm{D}$ anyon. Putting together Eqs. (D1) and (D2), we derive

$$
e^{i \Theta_{i, l}}=e^{2 \pi i N_{i} s_{\alpha}} .
$$

Thus, our problem reduces to computing the topological spin $s_{\alpha}$. To do this, we split $\alpha$ into two excitations: a surface anyon $\mathcal{X}$ and a vortex arch $\tilde{\alpha}$. We choose $\mathcal{X}$ to be any surface anyon that carries anyonic flux $\xi_{\mathcal{X}}=x_{i l}$, and we choose $\tilde{\alpha}$ to be any arch such that $\alpha$ can be written as a fusion product of $\tilde{\alpha}$ and $\mathcal{X}$. As in the previous sections, this splitting is designed so that $\tilde{\alpha}$ has a special property: if we shrink $\tilde{\alpha}$ to the surface, we get a superposition of different charge excitations (instead of more complicated surface anyons).

After this splitting, we apply the following result from 2D anyon theory:

$$
R_{\mathcal{X} \tilde{\alpha}}^{\alpha} R_{\tilde{\alpha} \mathcal{X}}^{\alpha}=e^{2 \pi i\left(s_{\alpha}-s_{\tilde{\alpha}}-s_{\mathcal{X}}\right)} \hat{I} .
$$

Here, $R_{\mathcal{X} \tilde{\alpha}}^{\alpha}$ is the $R$ symbol, so $R_{\mathcal{X} \tilde{\alpha}}^{\alpha} R_{\tilde{\alpha} \mathcal{X}}^{\alpha}$ describes the effect of a full braiding of $\mathcal{X}$ around $\tilde{\alpha}$ in the fusion channel $\alpha$. Comparing this relation with Eq. (D3), we derive

$$
e^{i \Theta_{i, l}}=e^{2 \pi i N_{i}\left(s_{\tilde{\alpha}}+s_{\mathcal{X}}\right)}\left(R_{\mathcal{X} \tilde{\alpha}}^{\alpha} R_{\tilde{\alpha} \mathcal{X}}^{\alpha}\right)^{N_{i}} .
$$

Now we evaluate the terms on the right-hand side of Eq. (D5). The quantity $s_{\mathcal{X}}$ can be computed as

$$
\begin{aligned}
e^{2 \pi i s_{\mathcal{X}}} & =e^{i \theta_{\xi_{\mathcal{X}}}} \\
& =e^{i \sum_{\mu}\left(x_{i l}^{\mu}\right)^{2} \Phi_{\mu}} e^{i \sum_{\mu<\nu} x_{i l}^{\mu} x_{i l}^{\nu} \Phi_{\mu \nu}},
\end{aligned}
$$

where the first equality follows from Eq. (15), and the second equality follows from $\xi_{\mathcal{X}}=x_{i l}$ together with Eq. (12). If we now raise this equation to the $N_{i}$ th power, the second term on the right-hand side drops out since $N_{i} x_{i l}^{\mu}$ is a multiple of $N_{\mu}$ and $N_{\mu} \Phi_{\mu \nu}$ is a multiple of $2 \pi$ according to the constraints Eqs. (27) and (21). Thus, we obtain 


$$
e^{2 \pi i N_{i} s_{\mathcal{X}}}=e^{i \sum_{\mu} N_{i}\left(x_{i l}^{\mu}\right)^{2} \Phi_{\mu}} .
$$

Next, consider the mutual braiding statistics term $\left(R_{\mathcal{X} \tilde{\alpha}}^{\alpha} R_{\tilde{\alpha} \mathcal{X}}^{\alpha}\right)^{N_{i}}$. This term is given by

$$
\left(R_{\mathcal{X} \tilde{\alpha}}^{\alpha} R_{\tilde{\alpha} \mathcal{X}}^{\alpha}\right)^{N_{i}}=\exp \left(i \sum_{\mu} \frac{N_{i}}{N^{i \mu}} x_{i l}^{\mu} \Omega_{i \mu}\right)
$$

according to Eqs. (48) and (49), with $i=j$.

The final piece to compute is $e^{2 \pi i N_{i} s_{\tilde{\alpha}}}$. We now argue that this term is trivial:

$$
e^{2 \pi i N_{i} s_{\tilde{\alpha}}}=1
$$

To prove this, recall that $\tilde{\alpha}$ has a special property: if we shrink $\tilde{\alpha}$ down to the surface, we get a superposition of charges rather than more complicated surface anyons. Let $-q$ be one of the charges that appears in this superposition, and let $\tilde{\alpha}^{\prime}$ be another vortex arch obtained by fusing $\tilde{\alpha}$ with $q$. Clearly $\tilde{\alpha}^{\prime}$, like $\tilde{\alpha}$, has the property that if we shrink it down to the surface, we get a superposition of charges. Furthermore, by construction, one of the charges that appears in this superposition is $q=0$, i.e., the vacuum. This means that if we shrink $\tilde{\alpha}^{\prime}$ down to the surface and then apply a projection operator that projects onto the $q=0$ state, then we can annihilate $\tilde{\alpha}^{\prime}$ locally.

Let us think about this property of $\tilde{\alpha}^{\prime}$ in a geometry where we fold the surface and straighten $\tilde{\alpha}^{\prime}$ as in Fig. 7. Then, if we view our system as two dimensional, the above property is equivalent to the statement that $\tilde{\alpha}^{\prime}$ can be annihilated locally at the edge of our system (where the edge runs along the folding axis).

At this point, we use a general result about 2D systems: any anyon excitation $a$ that can be annihilated locally at a gapped edge of a 2D system must have vanishing topological spin, $s_{a}=0$. This result was discussed in Ref. [59] in the case where the anyon $a$ has Abelian quasiparticle statistics, but we expect that this result holds in the nonAbelian case as well, and we assume this in what follows.

Assuming the above result, we deduce that $s_{\tilde{\alpha}^{\prime}}=0$. With this identity, it is now simple to derive Eq. (D9). First, we express $s_{\tilde{\alpha}}$ in terms of $s_{\tilde{\alpha}^{\prime}}$ :

$$
e^{2 \pi i s_{\tilde{\alpha}} \hat{I}}=e^{2 \pi i\left(s_{\tilde{\alpha}^{\prime}}+s_{q}\right)} R_{\tilde{\alpha}^{\prime} q}^{\tilde{\alpha}} R_{q \tilde{\alpha}^{\prime}}^{\tilde{\alpha}}
$$

Next, we note that the product $R_{\tilde{\alpha}^{\prime} q}^{\tilde{\alpha}} R_{q \tilde{\alpha}^{\prime}}^{\tilde{\alpha}}$ describes a full braiding of $q$ around $\tilde{\alpha}^{\prime}$ and, therefore, is an AharonovBohm phase of the form $e^{2 \pi i \text { (integer) } / N_{i}}$. At the same time, we have $s_{q}=s_{\tilde{\alpha}^{\prime}}=0$. Making these substitutions, we conclude that $s_{\tilde{\alpha}}$ must be a multiple of $1 / N_{i}$, which implies Eq. (D9).

The derivation is now complete: substituting Eqs. (D7), (D8), and (D9) into Eq. (D5), we derive the formula Eq. (30).

\section{APPENDIX E: SOME EQUATIONS FROM SEC. V}

In this appendix, we establish several equations that we use in the derivation of bulk-boundary correspondence. To be specific, we prove the commutation relations Eqs. (46) and (47), as well as the relations Eqs. (48) and (49), which involve taking the $N^{i j}$ th power of various braid matrices.

\section{Commutation relations}

To avoid repetition, we prove only the commutation relation Eq. (46); the derivation of the other commutation relation Eq. (47) follows similar arguments. We begin by rewriting Eq. (46) as

$$
W_{\tilde{\alpha} \tilde{\beta}}^{-1} W_{\mathcal{X} \tilde{\beta}}^{-1} W_{\tilde{\alpha} \tilde{\beta}} W_{\mathcal{X} \tilde{\beta}}=e^{i \sum_{\mu} x_{i l}^{\mu} \Omega_{i j \mu}} \hat{I} .
$$

Here, the braid matrices $W_{\tilde{\alpha} \tilde{\beta}}$ and $W_{\mathcal{X} \tilde{\beta}}$ are defined as in Eqs. (40) and (41), while $\tilde{\alpha}, \tilde{\beta}$ are vortex lines carrying unit type- $i$ and type- $j$ gauge flux, and $\mathcal{X}$ is a surface anyon carrying anyonic flux $x_{i l}$.

Next, we recall that braiding statistics is symmetric in the sense that braiding $X$ around $Y$ is topologically equivalent to braiding $Y$ around $X$. This means that $W_{\mathcal{X} \tilde{\beta}}=W_{\tilde{\beta} \mathcal{X}}$ and $W_{\tilde{\alpha} \tilde{\beta}}=W_{\tilde{\beta} \tilde{\alpha}}$. Therefore, instead of proving Eq. (E1), it suffices to prove

$$
W_{\tilde{\beta} \tilde{\alpha}}^{-1} W_{\tilde{\beta} \mathcal{X}}^{-1} W_{\tilde{\beta} \tilde{\alpha}} W_{\tilde{\beta} \mathcal{X}}=e^{i \sum_{\mu} x_{i l}^{\mu} \Omega_{i j \mu}} \hat{I} .
$$

To proceed further, we imagine splitting $\mathcal{X}$ into a collection of more "elementary" surface anyons $\left\{\mathcal{X}_{\mu}^{n}\right\}$. More specifically, we split $\mathcal{X}$ into $\left\{\mathcal{X}_{\mu}^{n}\right\}$, where each $\mathcal{X}_{\mu}^{n}$ carries unit type- $\mu$ anyonic flux. Here, the index $\mu$ runs from $1, \ldots, M$, while $n$ runs from $1, \ldots, x_{i l}^{\mu}$.

After this splitting, we decompose the process $W_{\tilde{\beta} \mathcal{X}}$ into a sequence of subprocesses in which $\tilde{\beta}$ is braided around each elementary anyon $\mathcal{X}_{\mu}^{n}$. Translating this into algebra, we obtain

$$
W_{\tilde{\beta} \mathcal{X}}=W_{\tilde{\beta} \mathcal{X}_{1}^{1}} \cdots W_{\tilde{\beta} \mathcal{X}_{M}^{x}},
$$

where we use the abbreviation $x \equiv x_{i l}^{M}$.

Given Eq. (E3), our task reduces to proving a commutation relation involving the elementary anyons $\mathcal{X}_{\mu}^{n}$ :

$$
W_{\tilde{\beta} \tilde{\alpha}}^{-1} W_{\tilde{\beta} \mathcal{X}_{\mu}^{n}}^{-1} W_{\tilde{\beta} \tilde{\alpha}} W_{\tilde{\beta} \mathcal{X}_{\mu}^{n}}=e^{i \Omega_{i j \mu} \hat{I}} .
$$

Indeed, it is easy to see that Eq. (E2) follows from Eqs. (E3) and (E4) since our decomposition $\mathcal{X} \rightarrow\left\{\mathcal{X}_{\mu}^{n}\right\}$ contains $x_{i l}^{\mu}$ anyons carrying unit type- $\mu$ anyonic flux.

To complete the proof, we now derive Eq. (E4). Consider the following braiding processes: 


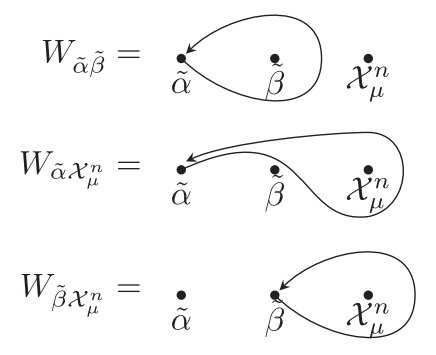

Now consider the product $W_{\tilde{\alpha} \chi_{\mu}^{n}} W_{\tilde{\alpha} \tilde{\beta}}$. We can see that this product corresponds to a process in which $\tilde{\alpha}$ is braided around $\tilde{\beta}$ and $\mathcal{X}_{\mu}^{n}$ as a whole. It follows that this product commutes with $W_{\tilde{\beta} \mathcal{X}_{\mu}^{n}}$, since the two braiding processes do not overlap. Therefore, we derive

$$
\left(W_{\tilde{\alpha} \mathcal{X}_{\mu}^{n}} W_{\tilde{\alpha} \tilde{\beta}}\right) W_{\tilde{\beta} \mathcal{X}_{\mu}^{n}}=W_{\tilde{\beta} \mathcal{X}_{\mu}^{n}}\left(W_{\tilde{\alpha} \mathcal{X}_{\mu}^{n}} W_{\tilde{\alpha} \tilde{\beta}}\right) .
$$

At the same time, we have the relation

$$
W_{\tilde{\beta} \mathcal{X}_{\mu}^{n}}^{-1} W_{\tilde{\alpha} \mathcal{X}_{\mu}^{n}}^{-1} W_{\tilde{\beta} \mathcal{X}_{\mu}^{n}} W_{\tilde{\alpha} \mathcal{X}_{\mu}^{n}}=e^{i \Omega_{i j \mu} \hat{I}},
$$

which follows from the definition of $\Omega_{i j \mu}$ and the property $W_{X Y}=W_{Y X}$ for any $X$ and $Y$. Combining Eqs. (E5) and (E6) and using $W_{\tilde{\alpha} \tilde{\beta}}=W_{\tilde{\beta} \tilde{\alpha}}$, straightforward algebra gives Eq. (E4).

\section{Relations with $N^{i j}$ th power}

We now derive the relation Eq. (48), which we reproduce below for convenience:

$$
\left(W_{\mathcal{X} \tilde{\beta}}\right)^{N^{i j}}=\exp \left(i \sum_{\mu} \frac{N^{i j}}{N^{j u}} x_{i l}^{\mu} \Omega_{j u}\right) \hat{I} .
$$

The other relation, Eq. (49), can be obtained in the same way, so we do not discuss it here.

To begin, we recall that braiding is symmetric, so it suffices to show that

$$
\left(W_{\tilde{\beta} \mathcal{X}}\right)^{N^{i j}}=\exp \left(i \sum_{\mu} \frac{N^{i j}}{N^{j u}} x_{i l}^{\mu} \Omega_{j u}\right) \hat{I} .
$$

Next, we split $\mathcal{X}$ into elementary anyons $\left\{\mathcal{X}_{\mu}^{n}\right\}$, as in the previous section. As before, each elementary anyon $\mathcal{X}_{\mu}^{n}$ carries unit type- $\mu$ anyonic flux and the index $\mu$ runs from $1, \ldots, M$, while $n$ runs from $1, \ldots, x_{i l}^{\mu}$.

We then decompose the process of braiding $\tilde{\beta}$ around $\mathcal{X}$ into a sequence of subprocesses in which $\tilde{\beta}$ is braided around each $\mathcal{X}_{\mu}^{n}$. Translating this into algebra gives

$$
W_{\tilde{\beta} \mathcal{X}}=W_{\tilde{\beta} \mathcal{X}_{1}^{1}} \cdots W_{\tilde{\beta} \mathcal{X}_{M}^{x}},
$$

where we use the abbreviation $x \equiv x_{i l}^{M}$.
Next, we claim that $W_{\tilde{\beta} \mathcal{X}_{\mu}^{n}}$ commutes with $W_{\tilde{\beta} \mathcal{X}_{\nu}^{m}}$ for arbitrary $n, m, \mu, \nu$. To see this, note that the following identity holds by the same reasoning as Eq. (E5):

$$
\left(W_{\mathcal{X}_{\nu}^{m} \tilde{\beta}} W_{\mathcal{X}_{\nu}^{m}} \mathcal{X}_{\mu}^{n}\right) W_{\mathcal{X}_{\mu}^{n} \tilde{\beta}}=W_{\mathcal{X}_{\mu}^{n} \tilde{\beta}}\left(W_{\mathcal{X}_{\nu}^{m} \tilde{\beta}} W_{\mathcal{X}_{\nu}^{m} \mathcal{X}_{\mu}^{n}}\right) .
$$

Furthermore, the mutual statistics of $\mathcal{X}_{\mu}^{n}$ and $\mathcal{X}_{\nu}^{m}$ is Abelian [see Eq. (14)], so $W_{\mathcal{X}_{\nu}^{m} \mathcal{X}_{\mu}^{n}}$ is proportional to the identity operator. It follows that $W_{\mathcal{X}_{\nu}^{m} \tilde{\beta}} W_{\mathcal{X}_{\mu}^{n} \tilde{\beta}}=W_{\mathcal{X}_{\mu}^{n} \tilde{\beta}} W_{\mathcal{X}_{\nu}^{m} \tilde{\beta}}$, so $W_{\tilde{\beta} \mathcal{X}_{\mu}^{n}}$ and $W_{\tilde{\beta} \mathcal{X}_{\nu}^{m}}$ commute.

Using the above commutativity property together with Eq. (E9), we derive the relation

$$
W_{\tilde{\beta} \mathcal{X}}^{N_{i j}}=W_{\tilde{\beta} \mathcal{X}_{1}^{1}}^{N_{i j}} \cdots W_{\tilde{\beta} \mathcal{X}_{M}^{x}}^{N_{i j}} .
$$

To proceed further, we make use of the following result:

$$
W_{\tilde{\beta} \mathcal{X}_{\mu}^{n}}^{N_{j}}=e^{i \vartheta_{\tilde{\beta} \mu} \hat{I}},
$$

where $\vartheta_{\tilde{\beta} \mu}$ is some phase that depends only on $\tilde{\beta}$ and $\mu$ and is independent of $n$.

Before establishing Eq. (E11), we now show that it implies our claim, Eq. (E8). To see this, we insert Eq. (E11) into Eq. (E10). Then using the fact that our decomposition $\mathcal{X} \rightarrow\left\{A_{\mu}^{n}\right\}$ contains $x_{i l}^{\mu}$ anyons carrying unit type- $\mu$ anyonic flux, we derive

$$
W_{\tilde{\beta} \mathcal{X}}^{N^{i j}}=\exp \left(i \sum_{\mu} \frac{N^{i j}}{N_{j}} x_{i l}^{\mu} \vartheta_{\tilde{\beta} \mu}\right) \hat{I} .
$$

At the same time, we know that

$$
W_{\tilde{\beta} \mathcal{X}_{\mu}^{n}}^{N^{j \mu}}=e^{i \Omega_{j \mu} \hat{I}}
$$

by the definition of $\Omega_{j \mu}$. Comparing Eqs. (E11) and (E13), we see that

$$
\Omega_{j \mu}=\frac{N^{j \mu}}{N_{j}} \vartheta_{\tilde{\beta} \mu} \quad(\bmod 2 \pi) .
$$

Therefore, we can rewrite Eq. (E12) as

$$
\begin{aligned}
W_{\tilde{\beta} \mathcal{X}}^{N^{i j}} & =\exp \left(i \sum_{\mu} \frac{N^{i j} x_{i l}^{\mu}}{N^{j \mu}} \frac{N^{j \mu}}{N_{j}} \vartheta_{\tilde{\beta} \mu}\right) \hat{I} \\
& =\exp \left(i \sum_{\mu} \frac{N^{i j} x_{i l}^{\mu}}{N^{j \mu}} \Omega_{j \mu}\right) \hat{I} .
\end{aligned}
$$

Here, in the second line, we use the fact that $\left(N^{i j} x_{i l}^{\mu} / N^{j \mu}\right)$ is an integer which follows from the general constraint Eq. (27) with a little algebra. This proves the claim, Eq. (E8). 
All that remains is to derive Eq. (E11). To this end, we observe that $W_{\tilde{\beta} \mathcal{X}_{n}^{n}}^{N_{j}}$ is the braid matrix for a process in which a vortex line $\tilde{\beta}$ is braided around a surface anyon $\mathcal{X}_{\mu}^{n}$ for $N_{j}$ times. This process is similar to the one discussed in Appendix A 1, and we can use similar methods to analyze it. In fact, we can essentially repeat the diagrammatic computation shown in Fig. 12 with the substitutions $\mathcal{X} \rightarrow \tilde{\beta}, \alpha \rightarrow \mathcal{X}_{\mu}^{n}$, and $N^{i \mu} \rightarrow N_{j}$. The first equation in Fig. 12 carries over without change. As for the second equation, we note that if we fuse $\tilde{\beta}$ with itself $N_{j}$ times, the only possible fusion products are surface anyons and charges, since $\tilde{\beta}$ carries gauge flux $\phi_{\tilde{\beta}}=\left(2 \pi / N_{j}\right) \mathbf{e}_{j}$. Therefore, in the second equation, the summation over charges $q$ is replaced by a summation over surface anyons and charges. The third equation also carries over with the only modification being that the Aharonov-Bohm phases $\exp \left(i q \cdot \phi_{\alpha}\right)$ are replaced by the statistical phase between these surface anyons or charges and $\mathcal{X}_{\mu}^{n}$. The latter depends on only the anyonic flux $\xi_{\mathcal{X}_{\mu}^{n}}=\epsilon_{\mu}$, but not on the anyon $\mathcal{X}_{\mu}^{n}$ itself. The last equation also holds, since we can group all the numerical factors into a single complex constant $C_{\tilde{\beta}, \epsilon_{\mu}}$. This constant must be a phase factor, since the braiding process is unitary. Thus, we can write $C_{\tilde{\beta}, \boldsymbol{\epsilon}_{\mu}}=e^{i \vartheta_{\tilde{\beta} \mu}}$ for some $\vartheta_{\tilde{\beta} \mu}$. This establishes Eq. (E11).

\section{APPENDIX F: DERIVING EQ. (D2)}

In this appendix, we derive Eq. (D2), which we reproduce for convenience:

$$
V|\psi\rangle=e^{-4 \pi i s_{\alpha}}|\psi\rangle .
$$

Let us recall the meaning of the different symbols in this equation: $|\psi\rangle$ is a particular state containing a vortex $\operatorname{arch} \alpha$ and its antiarch $\bar{\alpha}, V$ is the braid matrix associated with braiding $\alpha$ around $\bar{\alpha}$, and $s_{\alpha}$ is the topological spin of $\alpha$. Here, the precise definition of $s_{\alpha}$ involves folding the surface and straightening the vortex lines as in Fig. 7. After this folding and straightening, we view $\alpha$ as a 2D anyon and we define its topological spin $s_{\alpha}$ in the usual way.

To prove Eq. (F1) we use a special property of the state $|\psi\rangle$. To explain this property, imagine fusing together the two arches $\alpha$ and $\bar{\alpha}$. Since $\alpha$ and $\bar{\alpha}$ carry opposite gauge flux, this fusion process annihilates the vortex arches in the bulk, leaving behind a pair of surface excitations-one excitation at each end of the arches. Next, imagine that we fuse together the pair of surface excitations. The special property of $|\psi\rangle$ is that this second fusion process yields a superposition of charges rather than more complicated surface anyons. To prove this property, recall that the state $|\psi\rangle$ is constructed through a particular procedure: we take two bulk vortex loops $\alpha$ and $\bar{\alpha}$, which are linked to a third loop $\sigma$, and then we absorb them into the boundary and unwind $\sigma$ as in Fig. 5. Now, notice that if we fuse the loops $\alpha$ and $\bar{\alpha}$ in the bulk, i.e., before absorbing them to the surface, then we get a superposition of charge excitations. At the same time, it is not hard to argue that the deformation to the surface cannot change this property. Putting these two facts together, the above property of $|\psi\rangle$ follows immediately.

To understand the implications of this property, imagine that we fold the surface and then view $\alpha$ and $\bar{\alpha}$ as $2 \mathrm{D}$ anyons, as shown in Fig. 7. We can then think of the $\alpha \times \bar{\alpha}$ fusion process from a $2 \mathrm{D}$ point of view. Clearly, the different fusion outcomes are of the general form $C=(\mathcal{X}, \mathcal{Y})$, where $(\mathcal{X}, \mathcal{Y})$ denotes an excitation with a surface anyon $\mathcal{X}$ on the bottom surface and $\mathcal{Y}$ on the top surface (see Fig. 14). In this setup, the above property of $|\psi\rangle$ is equivalent to the statement that

$$
\xi_{\mathcal{X}}+\xi_{\mathcal{Y}}=0
$$

for any of the possible fusion outcomes $C$.

Equation (F2) is useful because it implies that all the fusion outcomes $C=(\mathcal{X}, \mathcal{Y})$ have vanishing topological spin; that is,

$$
s_{C}=0 .
$$

To see this, note that $C$ is the fusion product of $\mathcal{X} \equiv(\mathcal{X}, \mathbf{1})$ and $\mathcal{Y}^{\mathrm{op}} \equiv(\mathbf{1}, \mathcal{Y})$. Therefore, using a formula from the general algebraic theory [47] of anyons, we have

$$
e^{i 2 \pi s_{C} \hat{I}}=e^{i 2 \pi\left(s_{\mathcal{X}}+s_{\mathcal{Y}}{ }^{\text {op }}\right)} R_{\mathcal{X} \mathcal{Y}^{\circ \mathrm{p}}}^{C} R_{\mathcal{Y}^{\circ \mathrm{op} \mathcal{X}}}^{C},
$$

where $R_{\mathcal{X} \mathcal{Y}^{\text {op }}}^{C} R_{\mathcal{Y}^{\text {op }} \mathcal{X}}^{C}$ describes a full braiding of $\mathcal{X}$ and $\mathcal{Y}^{\text {op }}$ in the fusion channel $C$. Let us compute the different terms on the right-hand side. We have

$$
s_{\mathcal{X}}=s_{\xi_{\mathcal{X}}},
$$

by Eq. (15). Similarly,

$$
s_{\mathcal{Y}^{\mathrm{op}}}=-s_{\mathcal{Y}}=-s_{\xi_{\mathcal{Y}}}=-s_{-\xi_{\mathcal{Y}}}=-s_{\xi_{\mathcal{X}}},
$$

where the first equality follows from the fact that the anyons on the top and bottom surfaces have opposite statistics, the third equality follows from the relation $s_{x}=s_{-x}$, and the last equality follows from Eq. (F2). At the same time, it is clear that $R_{\mathcal{X} \mathcal{Y}^{\text {op }}}^{C} R_{\mathcal{Y}^{\text {op } \mathcal{X}}}^{C}=\hat{I}$ since $\mathcal{X}$ and

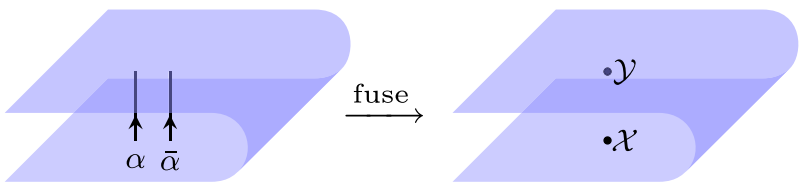

FIG. 14. Fusing vortex $\alpha$ and its antivortex $\bar{\alpha}$. The resulting doublet $(\mathcal{X}, \mathcal{Y})$ is viewed as a single anyon from the $2 \mathrm{D}$ slab point of view. 
$\mathcal{Y}^{\text {op }}$ live on different surfaces and therefore must have trivial braiding statistics with one another. Combining all these results, Eq. (F3) follows immediately.

Once we have Eq. (F3), we can easily derive Eq. (F1). Indeed, for each of the possible fusion outcomes $C$, we have

$$
\begin{aligned}
R_{\bar{\alpha} \alpha}^{C} R_{\alpha \bar{\alpha}}^{C} & =e^{i 2 \pi\left(s_{C}-s_{\alpha}-s_{\bar{\alpha}}\right) \hat{I}} \\
& =e^{-4 \pi i s_{\alpha} \hat{I}},
\end{aligned}
$$

where in the second line we use the fact that $s_{C}=0$ and $s_{\alpha}=s_{\bar{\alpha}}$. Since this equation holds for all possible fusion outcomes of $|\psi\rangle$, we immediately derive Eq. (F1).

\section{APPENDIX G: CONSTRAINTS ON THE SURFACE AND BULK DATA}

In this appendix, we derive the constraints Eqs. (23)-(26) on $\Omega_{i \mu}$ and $\Omega_{i j \mu}$. We also discuss the relationship between these surface constraints and previously known constraints on the bulk data.

\section{Constraints on $\Omega_{i \mu}$ and $\Omega_{i j \mu}$}

In the main text, we claim that the surface data satisfy the constraints Eqs. (23)-(26), which we reproduce here for convenience:

$$
\begin{array}{cc}
N_{i \mu} \Omega_{i \mu}=0 & (\bmod 2 \pi), \\
N_{i j \mu} \Omega_{i j \mu}=0 & (\bmod 2 \pi), \\
\Omega_{i j \mu}+\Omega_{j i \mu}=0 & (\bmod 2 \pi), \\
\Omega_{i i \mu}=0 & (\bmod 2 \pi) .
\end{array}
$$

We now prove these constraints.

To establish Eq. (G1), it suffices to show that $N_{\mu} \Omega_{i \mu}=0$ and $N_{i} \Omega_{i \mu}=0$. To prove $N_{\mu} \Omega_{i \mu}=0$, imagine braiding a vortex $\alpha$, carrying unit type- $i$ gauge flux, around a composite of $N_{\mu}$ identical anyons $\mathcal{X}$, each carrying a unit type- $\mu$ anyonic flux. Imagine further that we perform this braiding $N^{i \mu}$ times. We can compute the resulting statistical phase in two different ways. In the first approach, we note that the fusion product of $N_{\mu}$ anyons $\mathcal{X}$ yields a superposition of charge excitations, so the statistical phase must be 0 . In the second approach, we decompose the braiding process into a sequence of elementary processes in which $\alpha$ is braided around each $\mathcal{X}$ individually. From this alternate point of view, it is not hard to see that the total statistical phase is given by $N_{\mu} \Omega_{i \mu}$, since each $\mathcal{X}$ contributes a phase of $\Omega_{i \mu}$. Comparing these two calculations, we conclude that $N_{\mu} \Omega_{i \mu}=0$. Similar reasoning shows that $N_{i} \Omega_{i \mu}=0$, thus proving Eq. (G1).
The proof of Eq. (G2) is similar to Eq. (G1), so we do not present it here. As for Eq. (G3), this follows from the fact that the braiding process associated with $\Omega_{j i \mu}$ is identical to the process associated with $\Omega_{i j \mu}$, but performed in the reverse direction.

Finally, we need to show Eq. (G4). To this end, consider a state containing an anyon $\mathcal{X}$, a vortex $\alpha$, and its antivortex $\bar{\alpha}$. Suppose that $\mathcal{X}$ carries unit type- $\mu$ anyonic flux, while $\alpha$ carries unit type- $i$ gauge flux. Let us fold the surface and straighten the vortex lines as in Fig. 7, and imagine braiding $\alpha$ around $\mathcal{X}$ and then around $\bar{\alpha}$, and finally around $\mathcal{X}$ and $\bar{\alpha}$ in the opposite direction. We define the operators $V_{\alpha \mathcal{X}}$ and $V_{\alpha \bar{\alpha}}$ as braiding $\alpha$ around $\mathcal{X}$ and $\bar{\alpha}$, respectively. Then, the braiding process is algebraically given by the commutator $V_{\alpha \bar{\alpha}}^{-1} V_{\alpha \mathcal{X}}^{-1} V_{\alpha \bar{\alpha}} V_{\alpha \mathcal{X}}$.

To derive Eq. (G4), we evaluate this commutator in two different ways. In the first approach, we let the commutator act on an initial state $|\psi\rangle$ that satisfies the condition that if we shrink $\alpha$ and $\bar{\alpha}$ into the surface, we obtain a superposition of charges. Therefore, Eq. (D2) is applicable; i.e.,

$$
V_{\alpha \bar{\alpha}}|\psi\rangle=e^{-4 \pi i s_{\alpha}}|\psi\rangle .
$$

In addition, it is clear that the state $V_{\alpha \mathcal{X}}|\psi\rangle$ satisfies the same condition as $|\psi\rangle$, and therefore we also have

$$
V_{\alpha \bar{\alpha}} V_{\alpha \mathcal{X}}|\psi\rangle=e^{-4 \pi i s_{\alpha}} V_{\alpha \mathcal{X}}|\psi\rangle .
$$

Combining the above two relations, we immediately derive

$$
V_{\alpha \bar{\alpha}}^{-1} V_{\alpha \mathcal{X}}^{-1} V_{\alpha \bar{\alpha}} V_{\alpha \mathcal{X}}|\psi\rangle=|\psi\rangle .
$$

In the second approach, we compute the commutator using the same arguments that we use to derive Eq. (46). Proceeding as in that derivation, a straightforward calculation shows that the commutator is equal to $e^{-i \Omega_{i i \mu} \hat{I}}$. Comparing the results from the two approaches, we deduce that $\Omega_{i i \mu}=0$.

\section{Relationship with constraints on bulk data}

Reference [38] shows that, in the case of the group cohomology models [7], the bulk data satisfy the following constraints (modulo $2 \pi$ ):

$$
\begin{gathered}
\Theta_{i i, l}=2 \Theta_{i, l}, \\
\Theta_{i j, l}=\Theta_{j i, l}, \\
N_{i j l} \Theta_{i j, l}=0, \\
N_{i l} \Theta_{i, l}=0, \\
\frac{N^{i j l}}{N^{i j}} \Theta_{i j, l}+\frac{N^{i j l}}{N^{j l}} \Theta_{j l, i}+\frac{N^{i j l}}{N^{l i}} \Theta_{l i, j}=0,
\end{gathered}
$$




$$
\begin{gathered}
\frac{N^{i l}}{N_{i}} \Theta_{i, l}+\Theta_{i l, i}=0, \\
\Theta_{i, i}=0, \\
\Theta_{i j k, l}=\operatorname{sgn}(\hat{p}) \Theta_{\hat{p}(i) \hat{p}(j) \hat{p}(k), \hat{p}(l),}, \\
\Theta_{i i j, l}=0, \\
N_{i j k l} \Theta_{i j k, l}=0 .
\end{gathered}
$$

Here, $\hat{p}$ is a permutation of indices $i, j, k, l$ and $\operatorname{sgn}(\hat{p})$ is its signature. In addition, Ref. [38] proved the converse statement; that is, every solution to these constraints can be realized by a group cohomology model.

Interestingly, one can check that the bulk data defined by the bulk-boundary correspondence Eqs. (30)-(32) automatically obey the constraints Eqs. (G5)-(G14) as long as the surface data obey the constraints Eqs. (21)-(29). The proof is a straightforward mathematical exercise.

\section{APPENDIX H: EQUIVALENCE BETWEEN $\left\{\Omega_{i \mu}, \Omega_{i j \mu}\right\}$ AND $\left\{\boldsymbol{\omega} \in \boldsymbol{H}^{2}(\boldsymbol{G}, \boldsymbol{A})\right\}$}

In this appendix, we show that the mapping defined by Eqs. (69) and (71) is both injective and surjective. That is, we show that this mapping gives a one-to-one correspondence between the elements $\{\omega\}$ of $H^{2}(G, A)$ and the values of the surface data $\left\{\Omega_{i \mu}, \Omega_{i j \mu}\right\}$ that obey the constraints Eqs. (23)-(26).

To show the mapping is injective, we begin by counting the elements in $H^{2}(G, A)$. According to the Kunneth formula [7],

$$
H^{2}(G, A)=\prod_{i, \mu} \mathbb{Z}_{N_{i \mu}} \prod_{i<j, \mu} \mathbb{Z}_{N_{i j \mu}},
$$

where $G=\prod_{i=1}^{K} \mathbb{Z}_{N_{i}}$ and $A=\prod_{\mu=1}^{M} \mathbb{Z}_{N_{\mu}}$. Therefore, the number $\mathcal{N}$ of elements in $H^{2}(G, A)$ is equal to

$$
\mathcal{N}=\prod_{i, \mu} N_{i \mu} \prod_{i<j, \mu} N_{i j \mu} .
$$

Given this counting, we can establish injectivity if we can show that the image of the map defined by Eqs. (69) and (71) contains $\mathcal{N}$ distinct values of $\left\{\Omega_{i \mu}, \Omega_{i j \mu}\right\}$. To this end, consider the following class of 2-cocycles $\omega \in H^{2}(G, A)$, parametrized by integers $p_{i \nu}$ and $p_{i j \nu}$ :

$\omega^{\nu}(a, b)=\sum_{i} \frac{p_{i \nu}}{N_{i}}\left(a_{i}+b_{i}-\left[a_{i}+b_{i}\right]\right)+\sum_{i j} \frac{N_{\nu} p_{i j \nu}}{N_{i j \nu}} a_{i} b_{j}$.

(Here, $a, b \in G$.) It is not hard to check that Eq. (H3) satisfies the 2-cocycle condition Eq. (62). Inserting Eq. (H3) into Eqs. (69) and (71), we obtain

$$
\begin{aligned}
\Omega_{i \mu} & =\sum_{\nu} \frac{p_{i \nu} N_{\nu}}{N_{i \nu}} \Phi_{\mu \nu}, \\
\Omega_{i j \mu} & =\sum_{\nu} \frac{\left(p_{i j \nu}-p_{j i \nu}\right) N_{\nu}}{N_{i j \nu}} \Phi_{\mu \nu} .
\end{aligned}
$$

By varying $p_{i \mu}$ and $p_{i j \mu}$, it is easy to see that we can obtain $\mathcal{N}$ distinct values of $\left\{\Omega_{i \mu}, \Omega_{i j \mu}\right\}$. This proves that the mapping given by Eqs. (69) and (71) is injective.

To prove that the mapping is surjective, it suffices to show that there are only $\mathcal{N}$ values of $\left\{\Omega_{i \mu}, \Omega_{i j \mu}\right\}$ obeying the constraints Eqs. (23)-(26). The latter result can be established straightforwardly by constructing the most general solution to these constraints.

[1] Z.-C. Gu and X.-G. Wen, Tensor-Entanglement-Filtering Renormalization Approach and Symmetry-Protected Topological Order, Phys. Rev. B 80, 155131 (2009).

[2] F. Pollmann, A. M. Turner, E. Berg, and M. Oshikawa, Entanglement Spectrum of a Topological Phase in One Dimension, Phys. Rev. B 81, 064439 (2010).

[3] L. Fidkowski and A. Kitaev, Topological Phases of Fermions in One Dimension, Phys. Rev. B 83, 075103 (2011).

[4] X. Chen, Z.-C. Gu, and X.-G. Wen, Complete Classification of One-Dimensional Gapped Quantum Phases in Interacting Spin Systems, Phys. Rev. B 84, 235128 (2011).

[5] X. Chen, Z.-C. Gu, and X.-G. Wen, Classification of Gapped Symmetric Phases in One-Dimensional Spin Systems, Phys. Rev. B 83, 035107 (2011).

[6] N. Schuch, D. Pérez-García, and I. Cirac, Classifying Quantum Phases Using Matrix Product States and Projected Entangled Pair States, Phys. Rev. B 84, 165139 (2011).

[7] X. Chen, Z.-C. Gu, Z.-X. Liu, and X.-G. Wen, Symmetry Protected Topological Orders and the Group Cohomology of Their Symmetry Group, Phys. Rev. B 87, 155114 (2013).

[8] F. D. M. Haldane, Nonlinear Field Theory of Large-Spin Heisenberg Antiferromagnets: Semiclassically Quantized Solitons of the One-Dimensional Easy-Axis Néel State, Phys. Rev. Lett. 50, 1153 (1983).

[9] M.Z. Hasan and C. L. Kane, Colloquium: Topological Insulators, Rev. Mod. Phys. 82, 3045 (2010).

[10] X.-L. Qi and S.-C. Zhang, Topological Insulators and Superconductors, Rev. Mod. Phys. 83, 1057 (2011).

[11] C. L. Kane and E. J. Mele, $Z_{2}$ Topological Order and the Quantum spin Hall Effect, Phys. Rev. Lett. 95, 146802 (2005).

[12] C. Xu and J. E. Moore, Stability of the Quantum Spin Hall Effect: Effects of Interactions, Disorder, and $\mathbb{Z}_{2}$ Topology, Phys. Rev. B 73, 045322 (2006).

[13] C. Wu, B. A. Bernevig, and S.-C. Zhang, Helical Liquid and the Edge of Quantum Spin Hall Systems, Phys. Rev. Lett. 96, 106401 (2006).

[14] X. Chen, Z.-X. Liu, and X.-G. Wen, Two-Dimensional Symmetry-Protected Topological Orders and Their Protected Gapless Edge Eexcitations, Phys. Rev. B 84, 235141 (2011). 
[15] M. Levin and Z.-C. Gu, Braiding Statistics Approach to Symmetry-Protected Topological Phases, Phys. Rev. B 86, 115109 (2012).

[16] D. V. Else and C. Nayak, Classifying Symmetry-Protected Topological Phases through the Anomalous Action of the Symmetry on the Edge, Phys. Rev. B 90, 235137 (2014).

[17] A. Vishwanath and T. Senthil, Physics of Three-Dimensional Bosonic Topological Insulators: Surface-Ddeconfined Criticality and Quantized Magnetoelectric Effect, Phys. Rev. X 3, 011016 (2013).

[18] C. Wang and T. Senthil, Boson Topological Insulators: A Window into Highly Entangled Quantum Phases, Phys. Rev. B 87, 235122 (2013).

[19] F. J. Burnell, X. Chen, L. Fidkowski, and A. Vishwanath, Exactly Soluble Model of a Three-Dimensional SymmetryProtected Topological Phase of Bosons with Surface Topological Order, Phys. Rev. B 90, 245122 (2014).

[20] X. Chen, F. J. Burnell, A. Vishwanath, and L. Fidkowski, Anomalous Symmetry Fractionalization and Surface Topological Order, Phys. Rev. X 5, 041013 (2015).

[21] P. Bonderson, C. Nayak, and X.-L. Qi, A Time-Reversal Invariant Topological Phase at the Surface of a 3D Topological Insulator, J. Stat. Mech. (2013) P09016.

[22] C. Wang, A. C. Potter, and T. Senthil, Gapped Symmetry Preserving Surface State for the Electron Topological Insulator, Phys. Rev. B 88, 115137 (2013).

[23] X. Chen, L. Fidkowski, and A. Vishwanath, Symmetry Enforced Non-Abelian Topological Order at the Surface of a Topological Insulator, Phys. Rev. B 89, 165132 (2014).

[24] M. A. Metlitski, C. L. Kane, and M. P. A. Fisher, SymmetryRespecting Topologically Ordered Surface Phase of Three-Dimensional Electron Topological Insulators, Phys. Rev. B 92, 125111 (2015).

[25] L. Fidkowski, X. Chen, and A. Vishwanath, Non-Abelian Topological Order on the Surface of a 3D Topological Superconductor from an Exactly Solved Model, Phys. Rev. X 3, 041016 (2013).

[26] M. A. Metlitski, L. Fidkowski, X. Chen, and A. Vishwanath, Interaction Effects on 3D Topological Superconductors: Surface Topological Order from Vortex Condensation, the 16 Fold Way and Fermionic Kramers Doublets, arXiv: 1406.3032.

[27] C. Wang and T. Senthil, Interacting Fermionic Topological Insulators/Superconductors in Three Dimensions, Phys. Rev. B 89, 195124 (2014).

[28] X.-G. Wen, Topological Orders and Edge Excitations in Fractional Quantum Hall States, Adv. Phys. 44, 405 (1995).

[29] M. Levin and A. Stern, Classification and Analysis of Two-Dimensional Abelian Fractional Topological Insulators, Phys. Rev. B 86, 115131 (2012).

[30] Y.-M. Lu and A. Vishwanath, Theory and Classification of Interacting Integer Topological Phases in Two Dimensions: A Chern-Simons Approach, Phys. Rev. B 86, 125119 (2012).

[31] C. Xu and T. Senthil, Wave Functions of Bosonic Symmetry Protected Topological Phases, Phys. Rev. B 87, 174412 (2013).

[32] B. I. Halperin, Quantized Hall Conductance, CurrentCarrying Edge States, and the Existence of Extended States in a Two-Dimensional Disordered Potential, Phys. Rev. B 25, 2185 (1982).

[33] M. A. Metlitski, C. L. Kane, and M. P. A. Fisher, Bosonic Topological Insulator in Three Dimensions and the Statistical Witten Effect, Phys. Rev. B 88, 035131 (2013).

[34] T. Senthil, Symmetry-Protected Topological Phases of Quantum Matter, Annu. Rev. Condens. Matter Phys. 6, 299 (2015).

[35] H. Bombin, Topological Order with a Twist: Ising Anyons from an Abelian Model, Phys. Rev. Lett. 105, 030403 (2010).

[36] L. Fidkowski, N. H. Lindner, and A. Kitaev (unpublished).

[37] M. Barkeshli, P. Bonderson, M. Cheng, and Z. Wang, Symmetry, Defects, and Gauging of Topological Phases, arXiv: 1410.4540.

[38] C. Wang and M. Levin, Topological Invariants for Gauge Theories and Symmetry-Protected Topological Phases, Phys. Rev. B 91, 165119 (2015).

[39] J. B. Kogut, An Introduction to Lattice Gauge Theory and Spin Systems, Rev. Mod. Phys. 51, 659 (1979).

[40] C. Wang and M. Levin, Braiding Statistics of Loop Excitations in Three Dimensions, Phys. Rev. Lett. 113, 080403 (2014).

[41] S. Jiang, A. Mesaros, and Y. Ran, Generalized Modular Transformations in $(3+1) \mathrm{D}$ Topologically Ordered Phases and Triple Linking Invariant of Loop Braiding, Phys. Rev. X 4, 031048 (2014).

[42] To see this, recall that our gauging prescription maps gapped lattice boson models onto gapped gauge theories [15,38]. Therefore, if two lattice boson models can be connected without closing the gap, the corresponding gauge theories can also be connected without closing the gap and hence must have the same braiding statistics data.

[43] P. Etingof, N. Nikshych, and V. Ostrik, Fusion Categories and Homotopy Theory, Quantum Topol. 1, 209 (2010).

[44] A. Kapustin and R. Thorngren, Anomalies of Discrete Symmetries in Various Dimensions and Group Cohomology, arXiv:1404.3230.

[45] G. Y. Cho, J. C. Y. Teo, and S. Ryu, Conflicting Symmetries in Topologically Ordered Surface States of ThreeDimensional Bosonic Symmetry Protected Topological Phases, Phys. Rev. B 89, 235103 (2014).

[46] J. C. Wang, L. H. Santos, and X.-G. Wen, Bosonic Anomalies, Induced Fractional Quantum Numbers, and Degenerate Zero Modes: The Anomalous Edge Physics of Symmetry-Protected Topological States, Phys. Rev. B 91, 195134 (2015).

[47] A. Kitaev, Anyons in an Exactly Solved Model and Beyond, Ann. Phys. (Amsterdam) 321, 2 (2006).

[48] These loops differ from one another by the attachment of charge excitations.

[49] To make sense of braiding processes involving multiple vortex lines, we have to keep track of the other ends of the vortex lines.

[50] The reader may worry that the localized excitation in $|\psi\rangle$ might not have a definite anyon type - that is, it might be a linear superposition of different anyons; however, one can argue that this situation does not happen if the symmetry does not permute the anyons, as we assume here. 
[51] A. M. Essin and M. Hermele, Classifying Fractionalization: Symmetry Classification of Gapped $\mathbb{Z}_{2}$ Spin Liquids in Two Dimensions, Phys. Rev. B 87, 104406 (2013).

[52] Here, it is important to distinguish between strictly 2D systems and surfaces of 3D systems. In the context of strictly $2 \mathrm{D}$ systems, the data $\left\{F_{x y z}, R_{x y}, \omega \in H^{2}(G, A)\right\}$ needs to be supplemented by an additional piece of information, namely, an element of the cohomology group $\nu \in H^{3}(G, \mathrm{U}(1))[36,37,47]$. However, we believe that this additional piece of data is unnecessary for characterizing surfaces of 3D systems, at least if one defines the notion of "topologically equivalent" surfaces in an appropriate manner.

[53] The fundamental reason that this absorption or annihilation is possible is that the surface is symmetric by assumption. This symmetry guarantees that the bottoms of the vortex loops can be annihilated at the surface using local unitary operators.

[54] The reader may worry that the ambiguity in defining defect planes is fundamentally different from that for defect lines due to their larger dimensionality. This is not the case. If we compare two defect planes that correspond to the same group element $a$, they can differ at most by the attachment of a pointlike surface anyon that lies at the intersection of the end of the defect planes and the surface, since the bulk is short-range entangled.

[55] This identity follows easily from the cocycle condition Eq. (62).

[56] Here, we assume that the definitions of the surface data can be modified appropriately so that these quantities remain well defined whether or not the surface supports nonAbelian anyons.

[57] X. Chen, A. Tiwari, and S. Ryu, Bulk-Boundary Correspondence in $(3+1)$-Dimensional Topological Phases, arXiv:1509.04266.

[58] One subtlety is that, depending on how the deformation is performed, the arch obtained from the loop $\bar{\alpha}$ may or may not be the "antiarch" of that obtained from $\alpha$. However, we can always design our deformation so that this property holds.

[59] M. Levin, Protected Edge Modes without Symmetry, Phys. Rev. X 3, 021009 (2013). 